\title{
Conformational Transformations in Aromatic Nitroso Oxides
}

\author{
Alfia R. Yusupova, Rustam L. Safiullin, Sergey L. Khursan* \\ Ufa Institute of Chemistry of the Russian Academy of Sciences, 71 pr. Oktyabrya, Ufa, 450054 \\ Russian Federation

\section{E-mail: KhursanSL@gmail.com}

\section{S1. Geometrical parameters and frequencies of stretching vibrations of peroxynitrene}

This section describes in detail the choice of method of calculation. The computational results for the key geometrical and spectral parameters of trans- and cis-HNOO are presented in Table S1. As one can see, the O3LYP, M06, M11-L, and MN12-L functionals underestimate the O-O and $\mathrm{N}-\mathrm{O}$ bond lengths in the trans-form of nitroso oxide, whereas wB97, B971, B98, BMK, M06-2X, mPW1LYP, TPSSh, LC-wPBE, and wB97XD incorrectly reproduce the positions of absorption bands in the IR spectrum but well describe the geometrical parameters of the structure in question. Of the DFT methods studied, the M06-L, mPWPW91, OLYP, and HCTH functionals describe the structure and IR spectrum of trans-HNOO most reliably (Table S1).

Table S1. Geometrical parameters and frequencies of stretching vibrations of peroxynitrene

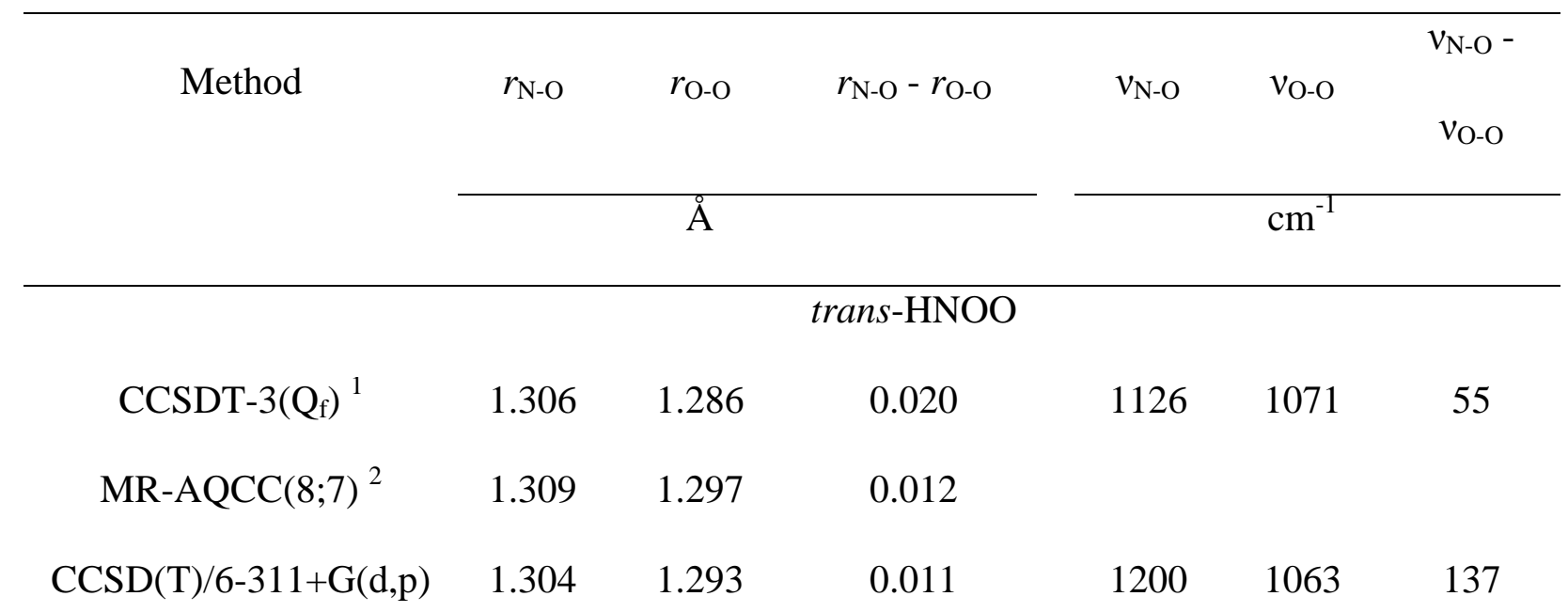




\section{CASSCF $(18 ; 13) /$}

\begin{tabular}{|c|c|c|c|c|c|c|}
\hline $6-311+G(d, p)^{2}$ & 1.317 & 1.297 & 0.020 & 1118 & 1044 & 72 \\
\hline Experiment $^{1}$ & & & & 1092.3 & 1054.5 & 38 \\
\hline M06 & 1.273 & 1.270 & 0.003 & 995 & 1196 & -201 \\
\hline M06-L & 1.282 & 1.278 & 0.004 & 1211 & 1136 & 75 \\
\hline wB97X & 1.295 & 1.277 & 0.018 & 1020 & 1230 & -210 \\
\hline M11-L & 1.252 & 1.249 & 0.003 & 1305 & 1208 & 97 \\
\hline MN12-L & 1.255 & 1.276 & -0.021 & 1329 & 1134 & 195 \\
\hline HCTH & 1.282 & 1.272 & 0.009 & 1191 & 1134 & 57 \\
\hline B971 & 1.292 & 1.288 & 0.004 & 988 & 1157 & -169 \\
\hline B98 & 1.294 & 1.287 & 0.007 & 986 & 1159 & -173 \\
\hline BMK & 1.300 & 1.283 & 0.017 & 1005 & 1241 & -236 \\
\hline M062X & 1.282 & 1.275 & 0.007 & 1017 & 1231 & -214 \\
\hline mPW1LYP & 1.304 & 1.292 & 0.012 & 970 & 1133 & -163 \\
\hline mPWPW91 & 1.297 & 1.294 & 0.003 & 1139 & 1086 & 53 \\
\hline OLYP & 1.294 & 1.285 & 0.009 & 1156 & 1115 & 41 \\
\hline O3LYP & 1.278 & 1.276 & 0.002 & 1001 & 1186 & -185 \\
\hline TPSSh & 1.301 & 1.295 & 0.006 & 955 & 1108 & -153 \\
\hline LC-wPBE & 1.297 & 1.273 & 0.024 & 1049 & 1258 & -209 \\
\hline wB97XD & 1.288 & 1.277 & 0.011 & 1015 & 1213 & -198 \\
\hline UB3LYP/6-31G(d) ${ }^{2}$ & 1.311 & 1.299 & 0.012 & 985 & 1147 & -162 \\
\hline \multicolumn{7}{|c|}{ cis-HNOO } \\
\hline MR-AQCC $(8 ; 7)^{2}$ & 1.288 & 1.318 & -0.030 & & & \\
\hline $\operatorname{CCSD}(\mathrm{T}) / 6-311+\mathrm{G}(\mathrm{d}, \mathrm{p})$ & 1.287 & 1.306 & -0.019 & 1224 & 1057 & 167 \\
\hline $\operatorname{CASSCF}(18 ; 13) /$ & 1.296 & 1.312 & -0.016 & 1131 & 1049 & 82 \\
\hline
\end{tabular}


$6-311+G(d, p)^{2}$

\begin{tabular}{ccccccc}
\hline M06-L & 1.262 & 1.296 & -0.034 & 1267 & 1096 & 171 \\
mPWPW91 & 1.277 & 1.313 & -0.036 & 1291 & 1048 & 243 \\
OLYP & 1.275 & 1.303 & -0.028 & 1209 & 1081 & 128 \\
HCTH & 1.263 & 1.290 & -0.027 & 1249 & 1095 & 154 \\
UB3LYP/6-31G(d) ${ }^{2}$ & 1.266 & 1.303 & -0.038 & 1321 & 1120 & 201 \\
\hline
\end{tabular}

Interatomic distances $r$ are expressed in Angstroms and the stretching vibration frequencies $v$ are in $\mathrm{cm}^{-1}$. The cc-pVTZ basis set was used in all the cases except where specified otherwise.

The methods that we chose show good applicability to calculations of the properties of trans-HNOO in terms of both selected criteria: bond lengths and position of absorption lines in the IR spectra. The use of these methods for calculation of the properties of cis-HNOO is also characterized by satisfactory agreement with the data of the reference methods: a correct order of $r$ and $v$ values is observed. The deviation of $r_{\mathrm{N}-\mathrm{O}}$ values from the reference methods by $0.01-$ $0.02 \AA$ and the corresponding overestimation of the N-O stretching vibration frequency by $c a$. $100 \mathrm{~cm}^{-1}$ should be noted. The length of the peroxide bond in the cis-form consistently increases in comparison with trans-HNOO, and all four methods give the same elongation of the O-O bond by $0.018 \AA$. The origin of the observed effect lies in non-valent orbital interaction that can occur only in cis-HNOO and can be described as partial electron density transfer from the lone electron pair of nitrogen to the antibonding orbital of the peroxide bond, $\mathrm{n}_{\mathrm{N}} \rightarrow \sigma^{*}{ }_{\mathrm{O}-\mathrm{O}}$ (Scheme S1). Partial population of the antibonding orbital of the $\mathrm{O}-\mathrm{O}$ bond destabilizes the peroxide bond and causes its elongation. ${ }^{3}$ For the same reason, additional $\pi$-bonding between $\mathrm{N}$ and $\mathrm{O}$ atoms occurs in cis-HNOO. It results in shortening of the $\mathrm{N}-\mathrm{O}$ bond and sign inversion of the $r_{\mathrm{N}-\mathrm{O}}-r_{\mathrm{O}-\mathrm{O}}$ difference (Table $\left.\mathrm{S} 1\right)$. 
Scheme S1
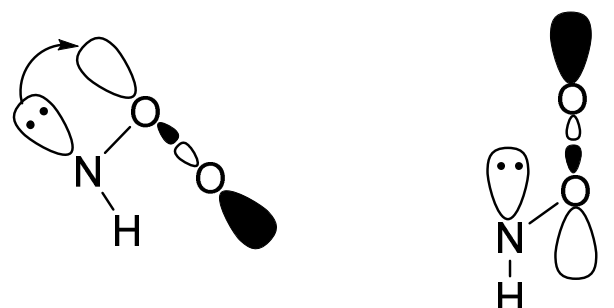

\section{S2. Trans-cis conformational transformation of peroxynitrene}

The computational results of reaction enthalpies $\Delta H^{\circ}$ and activation enthalpies $\Delta H^{\neq}$for transcis isomerization of peroxynitrene are presented in Table S2. Previously, the heat effect $\Delta H^{\circ}$ and activation energy $\Delta H^{\ddagger}$ for the trans-cis conformational transformation of peroxynitrene were found using high-level approximation MR-CISD(18;13)+Q. ${ }^{2}$ Taking these energies as the reference, we recalculated them using four selected functionals with both Dunning and Pople basis sets of triple valent splitting.

Table S2. Reaction enthalpies $\left(\Delta H^{\circ}\right)$ and activation enthalpies $\left(\Delta H^{\ddagger}\right)$ for trans-cis isomerization of peroxynitrene $(\mathrm{kJ} / \mathrm{mol})$

\begin{tabular}{|c|c|c|c|c|}
\hline \multirow{2}{*}{ Method } & $\Delta H^{\circ}$ & $\Delta H^{\ddagger}$ & $\Delta H^{\circ}$ & $\Delta H^{\neq}$ \\
\hline & \multicolumn{2}{|c|}{ cc-pVTZ } & \multicolumn{2}{|c|}{$6-311+G(d, p)$} \\
\hline MR-CISD $(18 ; 13)+\mathrm{Q}^{2}$ & -9.5 & 109.9 & -9.0 & 106.8 \\
\hline $\operatorname{CCSD}(\mathrm{T})$ & - & - & -12.2 & - \\
\hline M06-L & -14.4 & 106.5 & -12.7 & 108.4 \\
\hline mPWPW91 & -14.3 & 109.5 & -11.3 & 108.8 \\
\hline OLYP & -13.9 & 114.7 & -10.2 & 114.1 \\
\hline HCTH & -13.8 & 110.8 & -10.3 & 112.9 \\
\hline UB3LYP/6-31G(d) & & & -16.9 & 67.9 \\
\hline
\end{tabular}


Overall, good agreement is observed between the results of the DFT methods chosen and those of the reference method. All the approximations used correctly predict the higher stability of the cis-isomer of peroxynitrene. In fact, if the Pople basis set is used, the stability of cis-HNOO in comparison with the trans-isomer is overestimated by only $1 \mathrm{~kJ} / \mathrm{mol}(\mathrm{OLYP}, \mathrm{HCTH})$ and the conformation barrier is overestimated by $2 \mathrm{~kJ} / \mathrm{mol}$ (M06-L, mPWPW91). For comparison, Tables 1 and 2 show the results of calculations using the popular B3LYP functional. One can see that this method agrees with the reference method to within $0.01 \AA$ as concerns bond lengths. However, it poorly reproduces the vibrational spectrum of $\mathrm{HNOO}$ and considerably underestimates (by $40 \mathrm{~kJ} / \mathrm{mol}$ ) the activation barrier $\Delta H^{\neq}$of the conformational transition of trans-HNOO to the cis-isomer, which confirms the conclusion (see above) that it is undesirable to use hybrid methods for studies on the properties of nitroso oxides. Thus, to simulate conformational transformations of arylnitroso oxides, four density functionals were selected (M06-L, mPWPW91, OLYP, HCTH) that provide good agreement with the results of high-level calculations of the properties of peroxynitrene HNOO.

\section{S3. XYZ coordinates of the stationary points of HNOO; gas phase}




\section{cis-HNOO}

\section{M06-L/ cc-pVTZ}

\begin{tabular}{lrrr}
$\mathrm{O}$ & -1.095082000 & -0.227763000 & 0.000000000 \\
$\mathrm{O}$ & 0.000000000 & 0.465582000 & 0.000000000 \\
$\mathrm{~N}$ & 1.123011000 & -0.111008000 & 0.000000000 \\
$\mathrm{H}$ & 0.899584000 & -1.125499000 & 0.000000000 \\
\multicolumn{2}{l}{ mPWPW91/ cc-pVTZ }
\end{tabular}

$\begin{array}{llll}\text { O } & -1.108530000 & -0.232820000 & 0.000000000\end{array}$

$\begin{array}{llll}\text { O } & 0.000000000 & 0.470942000 & 0.000000000\end{array}$

$\begin{array}{llll}\mathrm{N} & 1.137082000 & -0.110339000 & 0.000000000\end{array}$

H $\quad 0.908667000 \quad-1.132606000 \quad 0.000000000$

\section{OLYP/ cc-pVTZ}
$\begin{array}{llll}\text { O } & -1.106867000 & -0.221781000 & 0.000000000\end{array}$
$\begin{array}{llll}\text { O } & 0.000000000 & 0.464925000 & 0.000000000\end{array}$
$\begin{array}{llll}\mathrm{N} & 1.134854000 & -0.115769000 & 0.000000000\end{array}$
H $\quad 0.910955000 \quad-1.134774000 \quad 0.000000000$

\section{HCTH/ cc-pVTZ}
$\begin{array}{llll}\text { O } & -1.097373000 & -0.217889000 & 0.000000000\end{array}$
$\begin{array}{llll}\text { O } & 0.000000000 & 0.459584000 & 0.000000000\end{array}$
$\begin{array}{llll}\mathrm{N} & 1.124473000 & -0.114642000 & 0.000000000\end{array}$
$\begin{array}{llll}\mathrm{H} & 0.907669000 & -1.131068000 & 0.000000000\end{array}$ 


\section{trans-HNOO}

\section{M06-L/ cc-pVTZ}

$\begin{array}{lrrr}\mathrm{O} & 1.133808000 & -0.182402000 & -0.000009000 \\ \mathrm{O} & 0.003094000 & 0.414227000 & 0.000007000 \\ \mathrm{~N} & -1.045801000 & -0.322834000 & 0.000004000 \\ \mathrm{H} & -1.774609000 & 0.405232000 & -0.000008000\end{array}$ mPWPW91/ cc-pVTZ

$\begin{array}{llll}\text { O } & 1.146880000 & -0.184691000 & 0.000003000\end{array}$

$\begin{array}{llll}\text { O } & 0.002633000 & 0.419316000 & -0.000003000\end{array}$

$\mathrm{N} \quad-1.057888000 \quad-0.326602000 \quad-0.000001000$

$\begin{array}{llll}\mathrm{H} & -1.790888000 & 0.409208000 & 0.000005000\end{array}$

\section{OLYP/ cc-pVTZ}

$\begin{array}{llll}\text { O } & 1.142003000 & -0.183788000 & 0.000002000\end{array}$

$\begin{array}{llll}\text { O } & 0.005067000 & 0.416054000 & -0.000002000\end{array}$

$\mathrm{N} \quad-1.056385000 \quad-0.324655000 \quad-0.000001000$

$\begin{array}{llll}\mathrm{H} & -1.781860000 & 0.414454000 & 0.000004000\end{array}$

\section{HCTH/ cc-pVTZ}

\begin{tabular}{lrrr}
$\mathrm{O}$ & 1.132417000 & -0.180967000 & 0.000004000 \\
$\mathrm{O}$ & 0.005681000 & 0.410029000 & -0.000003000 \\
$\mathrm{~N}$ & -1.047449000 & -0.320616000 & -0.000001000 \\
$\mathrm{H}$ & -1.772642000 & 0.411816000 & 0.000006000 \\
\multicolumn{2}{|l}{ ts (cis-trans) -HNOO } &
\end{tabular}

\section{M06-L/ cc-pVTZ}

$\begin{array}{lrrr}\mathrm{O} & 1.125527000 & -0.236836000 & -0.003512000 \\ \mathrm{O} & 0.023520000 & 0.440796000 & 0.046498000 \\ \mathrm{~N} & -1.079594000 & -0.159041000 & -0.138700000 \\ \mathrm{H} & -1.635214000 & -0.518390000 & 0.627011000\end{array}$
mPWPW91/ cc-pVTZ
O $\quad 1.158730000$
$-0.234449000 \quad-0.002538000$
$\begin{array}{llll}\text { O } & 0.001298000 & 0.447621000 & 0.046367000\end{array}$ 


$$
\begin{array}{llll}
\mathrm{N} & -1.087141000 & -0.166529000 & -0.137837000 \\
\mathrm{H} & -1.670236000 & -0.539668000 & 0.614225000
\end{array}
$$

\section{OLYP/ cc-pVTZ}

$\begin{array}{lrrr}\mathrm{O} & 1.145221000 & -0.234547000 & -0.002687000 \\ \mathrm{O} & 0.010851000 & 0.443507000 & 0.045826000 \\ \mathrm{~N} & -1.085293000 & -0.164107000 & -0.138590000 \\ \mathrm{H} & -1.651526000 & -0.522933000 & 0.625015000\end{array}$

\section{HCTH/ cc-pVTZ}

$\begin{array}{lrrr}\mathrm{O} & 1.073670000 & -0.309648000 & -0.002486000 \\ \mathrm{O} & 0.108453000 & 0.537824000 & 0.024121000 \\ \mathrm{~N} & -1.152821000 & -0.206729000 & -0.146058000 \\ \mathrm{H} & -1.387239000 & -0.378303000 & 0.849328000\end{array}$

S4. XYZ coordinates of the $\mathrm{C}_{6} \mathrm{H}_{5} \mathrm{NOO}$ stationary points; gas phase

$$
\text { cis- } \mathrm{C}_{6} \mathrm{H}_{5} \mathrm{NOO}
$$

$\operatorname{CCSD}(T) / 6-311+G(d, p)$
$\begin{array}{llll}\text { O } & -2.512490000 & 0.947440000 & 0.000000000\end{array}$
O $\quad-2.465940000 \quad-0.373740000 \quad 0.000000000$
$\mathrm{N} \quad-1.337160000 \quad-1.028700000 \quad 0.000000000$
C $\quad-0.106550000 \quad-0.360220000 \quad 0.000000000$
C $\quad 0.953010000 \quad-1.309800000 \quad 0.000000000$
C $\quad 2.283110000 \quad-0.900660000 \quad 0.000000000$
C $\quad 2.586840000 \quad 0.473010000 \quad 0.000000000$
$\begin{array}{llll}\text { C } & 1.551870000 & 1.417840000 & 0.000000000\end{array}$
C $\quad 0.205540000 \quad 1.027690000 \quad 0.000000000$
$\begin{array}{llll}\mathrm{H} & 0.685540000 & -2.363730000 & 0.000000000\end{array}$
$\begin{array}{llll}\mathrm{H} & 3.079660000 & -1.641050000 & 0.000000000\end{array}$
$\begin{array}{llll}\mathrm{H} & 3.624030000 & 0.802180000 & 0.000000000\end{array}$
$\mathrm{H} \quad 1.788440000 \quad 2.479740000 \quad 0.000000000$
$\begin{array}{llll}\mathrm{H} & -0.577000000 & 1.769060000 & 0.000000000\end{array}$ 


\section{M06-L/6-311+G(d,p)}

$\begin{array}{lrrr}\text { C } & 0.000000000 & 0.407096000 & 0.000000000 \\ \text { C } & 0.300030000 & -0.979212000 & 0.000000000 \\ \text { C } & -0.744599000 & -1.890601000 & 0.000000000 \\ \text { C } & -2.072068000 & -1.472097000 & 0.000000000 \\ \text { C } & -2.376987000 & -0.109636000 & 0.000000000 \\ \text { C } & -1.356553000 & 0.816743000 & 0.000000000 \\ \text { N } & 0.827679000 & 1.511796000 & 0.000000000 \\ \text { O } & 2.111869000 & 1.404963000 & 0.000000000 \\ \text { O } & 2.730443000 & 0.238358000 & 0.000000000 \\ \text { H } & 1.324342000 & -1.308681000 & 0.000000000 \\ \text { H } & -0.515646000 & -2.951098000 & 0.000000000 \\ \text { H } & -2.871933000 & -2.205986000 & 0.000000000 \\ \text { H } & -3.409906000 & 0.220487000 & 0.000000000 \\ \text { H } & -1.558049000 & 1.882381000 & 0.000000000 \\ \text { mPWPW91/6-311+G(d,p) } & \end{array}$

$\begin{array}{llll}\text { C } & 0.000000000 & 0.414215000 & 0.000000000\end{array}$

$\begin{array}{llll}\text { C } & 0.284549000 & -0.983618000 & 0.000000000\end{array}$

$\begin{array}{llll}\text { C } & -0.776890000 & -1.888538000 & 0.000000000\end{array}$

C $\quad-2.106969000 \quad-1.449954000 \quad 0.000000000$

$\begin{array}{llll}\text { C } & -2.396056000 & -0.075513000 & 0.000000000\end{array}$

$\begin{array}{llll}\text { C } & -1.359190000 & 0.845454000 & 0.000000000\end{array}$

$\begin{array}{llll}\mathrm{N} & 0.852105000 & 1.506971000 & 0.000000000\end{array}$

$\begin{array}{llll}\text { O } & 2.148991000 & 1.379345000 & 0.000000000\end{array}$

$\begin{array}{llll}\text { O } & 2.764748000 & 0.189178000 & 0.000000000\end{array}$

$\mathrm{H} \quad 1.313083000 \quad-1.321800000 \quad 0.000000000$

H $\quad-0.559292000 \quad-2.957253000 \quad 0.000000000$

$\begin{array}{llll}\mathrm{H} & -2.919995000 & -2.177339000 & 0.000000000\end{array}$

$\mathrm{H} \quad-3.430507000 \quad 0.268775000 \quad 0.000000000$

$\mathrm{H} \quad-1.550600000 \quad 1.918372000 \quad 0.000000000$ 


\section{OLYP/6-311+G(d,p)}

$\begin{array}{lrrr}\mathrm{C} & 0.000000000 & 0.407219000 & 0.000000000 \\ \mathrm{C} & 0.279622000 & -0.991085000 & 0.000000000 \\ \mathrm{C} & -0.781622000 & -1.895704000 & 0.000000000 \\ \mathrm{C} & -2.111340000 & -1.457174000 & 0.000000000 \\ \mathrm{C} & -2.397539000 & -0.083064000 & 0.000000000 \\ \mathrm{C} & -1.359618000 & 0.837257000 & 0.000000000 \\ \mathrm{~N} & 0.852557000 & 1.501305000 & 0.000000000 \\ \mathrm{O} & 2.145896000 & 1.389116000 & 0.000000000 \\ \mathrm{O} & 2.782260000 & 0.223270000 & 0.000000000 \\ \mathrm{H} & 1.301822000 & -1.337482000 & 0.000000000 \\ \mathrm{H} & -0.564366000 & -2.962117000 & 0.000000000 \\ \mathrm{H} & -2.923330000 & -2.182253000 & 0.000000000 \\ \mathrm{H} & -3.429168000 & 0.262393000 & 0.000000000 \\ \mathrm{H} & -1.555132000 & 1.906540000 & 0.000000000\end{array}$

\section{HCTH/6-311+G(d,p)}

$\begin{array}{lrrr}\text { C } & 0.000000000 & 0.405011000 & 0.000000000 \\ \text { C } & 0.283910000 & -0.986278000 & 0.000000000 \\ \text { C } & -0.768533000 & -1.892484000 & 0.000000000 \\ \text { C } & -2.094647000 & -1.461992000 & 0.000000000 \\ \text { C } & -2.385565000 & -0.094952000 & 0.000000000 \\ \text { C } & -1.355871000 & 0.825953000 & 0.000000000 \\ \text { N } & 0.844941000 & 1.496735000 & 0.000000000 \\ \text { O } & 2.126501000 & 1.392708000 & 0.000000000 \\ \text { O } & 2.764085000 & 0.243224000 & 0.000000000 \\ \text { H } & 1.304936000 & -1.329199000 & 0.000000000 \\ \mathrm{H} & -0.546503000 & -2.955453000 & 0.000000000 \\ \text { H } & -2.901514000 & -2.189228000 & 0.000000000 \\ \mathrm{H} & -3.416339000 & 0.245545000 & 0.000000000 \\ \mathrm{H} & -1.555613000 & 1.892179000 & 0.000000000\end{array}$




\section{trans- $\mathrm{C}_{6} \mathrm{H}_{5} \mathrm{NOO}$}

$\operatorname{CCSD}(\mathrm{T}) / 6-311+G(d, p)$
$\begin{array}{llll}\text { O } & -3.421950000 & -0.063850000 & 0.000000000\end{array}$
$\begin{array}{llll}\text { O } & -2.142270000 & -0.351070000 & 0.000000000\end{array}$
$\begin{array}{llll}\mathrm{N} & -1.339940000 & 0.693550000 & 0.000000000\end{array}$
$\begin{array}{llll}\text { C } & -0.004440000 & 0.257190000 & 0.000000000\end{array}$
C $\quad 0.427890000 \quad-1.090870000 \quad 0.000000000$
C $\quad 1.796410000 \quad-1.366080000 \quad 0.000000000$
$\begin{array}{llll}\text { C } & 2.738040000 & -0.319770000 & 0.000000000\end{array}$
$\begin{array}{llll}\text { C } & 2.307090000 & 1.015530000 & 0.000000000\end{array}$
$\begin{array}{llll}\text { C } & 0.938980000 & 1.305740000 & 0.000000000\end{array}$
$\begin{array}{llll}\mathrm{H} & -0.298550000 & -1.897560000 & 0.000000000\end{array}$
$\begin{array}{llll}\mathrm{H} & 2.134450000 & -2.400100000 & 0.000000000\end{array}$
H $\quad 3.801600000 \quad-0.548730000 \quad 0.000000000$
$\begin{array}{llll}\mathrm{H} & 3.032400000 & 1.825910000 & 0.000000000\end{array}$
$\begin{array}{llll}\mathrm{H} & 0.572880000 & 2.329730000 & 0.000000000\end{array}$

M06-L/6-311+G(d,p)
$\begin{array}{llll}\text { O } & -1.400186000 & -0.620922000 & 0.000571000\end{array}$
$\begin{array}{llll}\text { O } & -2.254376000 & 0.349657000 & -0.000284000\end{array}$
$\mathrm{N} \quad-3.518413000 \quad 0.001688000 \quad-0.000277000$
$\begin{array}{llll}\text { C } & -0.079840000 & -0.181189000 & 0.000194000\end{array}$
C $\quad 0.816907000 \quad-1.274465000 \quad 0.000215000$
$\begin{array}{llll}\text { C } & 0.388296000 & -2.271277000 & 0.000398000\end{array}$
C $\quad 2.188630000 \quad-1.076802000 \quad-0.000063000$
C $\quad 2.863227000 \quad-1.927428000 \quad-0.000104000$
C $\quad 2.686017000 \quad 0.228137000 \quad-0.000214000$
$\begin{array}{llll}\mathrm{H} & 3.757666000 & 0.406173000 & -0.000402000\end{array}$
$\begin{array}{llll}\mathrm{H} & 1.805988000 & 1.313265000 & -0.000102000\end{array}$
$\begin{array}{llll}\mathrm{H} & 2.209844000 & 2.322379000 & -0.000043000\end{array}$
$\begin{array}{llll}\mathrm{H} & 0.417633000 & 1.157319000 & 0.000066000\end{array}$ 


$\begin{array}{lrrr}\text { H } & -0.247435000 & 2.008250000 & 0.000097000 \\ \text { mPWPW91/6-311+G(d,p) } & \\ \text { N } & -1.401018000 & -0.687049000 & 0.000079000 \\ \text { O } & -2.222476000 & 0.337118000 & -0.000010000 \\ \text { O } & -3.506226000 & 0.053222000 & 0.000160000 \\ \text { C } & -0.077574000 & -0.251738000 & 0.000014000 \\ \text { C } & 0.864525000 & -1.307267000 & 0.000069000 \\ \text { H } & 0.492893000 & -2.331831000 & 0.000153000 \\ \text { C } & 2.228712000 & -1.024557000 & 0.000010000 \\ \text { H } & 2.951302000 & -1.840954000 & 0.000049000 \\ \text { C } & 2.668138000 & 0.304903000 & -0.000098000 \\ \text { H } & 3.735844000 & 0.526599000 & -0.000143000 \\ \text { C } & 1.734429000 & 1.356456000 & -0.000147000 \\ \text { H } & 2.082867000 & 2.389851000 & -0.000228000 \\ \text { C } & 0.369312000 & 1.094318000 & -0.000092000 \\ \text { H } & -0.351415000 & 1.910265000 & -0.000128000\end{array}$

\section{OLYP/6-311+G(d,p)}
$\begin{array}{llll}\text { O } & -1.405405000 & -0.679280000 & 0.000063000\end{array}$
$\begin{array}{llll}\text { O } & -2.229381000 & 0.336226000 & -0.000015000\end{array}$
$\begin{array}{llll}\mathrm{N} & -3.503073000 & 0.051750000 & 0.000186000\end{array}$
$\begin{array}{llll}\text { C } & -0.080677000 & -0.245207000 & 0.000007000\end{array}$
C $\quad 0.858560000 \quad-1.304156000 \quad 0.000065000$
$\begin{array}{llll}\text { C } & 0.487407000 & -2.326041000 & 0.000144000\end{array}$
$\begin{array}{llll}\text { C } & 2.224120000 & -1.029086000 & 0.000011000\end{array}$
$\begin{array}{llll}\text { C } & 2.939798000 & -1.848621000 & 0.000051000\end{array}$
$\begin{array}{llll}\text { C } & 2.671934000 & 0.297076000 & -0.000093000\end{array}$
$\begin{array}{llll}\mathrm{H} & 3.738670000 & 0.512405000 & -0.000133000\end{array}$
$\begin{array}{llll}\mathrm{H} & 1.743693000 & 1.352381000 & -0.000145000\end{array}$
$\mathrm{H} \quad 2.095824000 \quad 2.382179000 \quad-0.000225000$
$\begin{array}{llll}\mathrm{H} & 0.377088000 & 1.097619000 & -0.000097000\end{array}$ 
$\mathrm{H} \quad-0.332544000 \quad 1.919472000 \quad-0.000138000$

\section{HCTH/6-311+G(d,p)}

\begin{tabular}{|c|c|c|c|}
\hline $\mathrm{N}$ & -1.401113000 & -0.671712000 & 0.000101000 \\
\hline $\mathrm{O}$ & -2.219273000 & 0.331069000 & -0.000005000 \\
\hline $\mathrm{O}$ & -3.480054000 & 0.052400000 & 0.000123000 \\
\hline $\mathrm{C}$ & -0.082102000 & -0.243952000 & 0.000024000 \\
\hline $\mathrm{C}$ & 0.852860000 & -1.298371000 & 0.000074000 \\
\hline $\mathrm{H}$ & 0.480952000 & -2.317525000 & 0.000160000 \\
\hline $\mathrm{C}$ & 2.212915000 & -1.025385000 & 0.000006000 \\
\hline $\mathrm{H}$ & 2.926743000 & -1.843257000 & 0.000041000 \\
\hline $\mathrm{C}$ & 2.659365000 & 0.295152000 & -0.000103000 \\
\hline $\mathrm{H}$ & 3.723784000 & 0.509621000 & -0.000154000 \\
\hline $\mathrm{C}$ & 1.735458000 & 1.346286000 & -0.000145000 \\
\hline $\mathrm{H}$ & 2.087155000 & 2.373654000 & -0.000224000 \\
\hline $\mathrm{C}$ & 0.374431000 & 1.092747000 & -0.000082000 \\
\hline $\mathrm{H}$ & -0.333790000 & 1.912879000 & -0.000111000 \\
\hline
\end{tabular}

M06-L/6-311+G(d,p)
C $\quad-0.138003000$
$-0.426028000$
$-0.125805000$
C $\quad 0.129843000$
$0.973416000 \quad-0.202166000$
C $\quad 1.426730000$
$1.424099000 \quad-0.092639000$
C $\quad 2.486732000$
0.528306000
0.092593000
C $\quad 2.239184000 \quad-0.847621000$
0.169943000
C $\quad 0.953777000$
$-1.326650000$
0.064635000
$\mathrm{N}$
$-1.337074000$
$-1.021910000$
$-0.213699000$
$\begin{array}{llll}\text { O } & -2.386920000 & -0.001779000 & -0.436225000\end{array}$
$\begin{array}{llll}\text { O } & -2.796400000 & 0.510044000 & 0.678508000\end{array}$
$\begin{array}{llll}\mathrm{H} & -0.688005000 & 1.668141000 & -0.343795000\end{array}$
H $\quad 1.628484000 \quad 2.488712000 \quad-0.151536000$
$\begin{array}{llll}\mathrm{H} & 3.501719000 & 0.902403000 & 0.176674000\end{array}$ 


$\begin{array}{lrll}\text { H } & 3.062967000 & -1.538554000 & 0.314292000 \\ \text { H } & 0.731341000 & -2.386592000 & 0.122631000 \\ \text { mPWPW91/6-311+G(d,p) } & \\ \text { C } & -0.135052000 & -0.430033000 & -0.127869000 \\ \text { C } & 0.133782000 & 0.977465000 & -0.209789000 \\ \text { C } & 1.437480000 & 1.433151000 & -0.098980000 \\ \text { C } & 2.504505000 & 0.534539000 & 0.094278000 \\ \text { C } & 2.256867000 & -0.848993000 & 0.177151000 \\ \text { C } & 0.964948000 & -1.333992000 & 0.069728000 \\ \text { N } & -1.336408000 & -1.034771000 & -0.227221000 \\ \text { O } & -2.421062000 & -0.012198000 & -0.433151000 \\ \text { O } & -2.819560000 & 0.524998000 & 0.688840000 \\ \text { H } & -0.687518000 & 1.674725000 & -0.358419000 \\ \mathrm{H} & 1.638586000 & 2.503195000 & -0.164282000 \\ \mathrm{H} & 3.523867000 & 0.911890000 & 0.179976000 \\ \mathrm{H} & 3.085058000 & -1.542211000 & 0.327915000 \\ \mathrm{H} & 0.744643000 & -2.399424000 & 0.132728000\end{array}$

\section{OLYP/6-311+G(d,p)}
C $\quad-0.134415000$
$-0.420211000$
$-0.128007000$
C $\quad 0.150538000$
0.984629000
$-0.209528000$
C $\quad 1.457975000$
$1.429489000 \quad-0.098671000$
C $\quad 2.517729000$
0.523835000
0.097048000
C $\quad 2.257655000 \quad-0.856577000$
0.178939000
C $\quad 0.961799000$
$-1.330683000$
0.068724000
$\mathrm{N}$
$-1.335594000$
$-1.025408000$
$-0.235719000$
O $\quad-2.444479000$
$-0.016337000 \quad-0.422824000$
O $\quad-2.840716000$
$0.524419000 \quad 0.683449000$
$\begin{array}{llll}\mathrm{H} & -0.657356000 & 1.691508000 & -0.363108000\end{array}$
$\mathrm{H} \quad 1.666547000 \quad 2.495686000 \quad-0.166182000$
H $\quad 3.537931000$
0.892214000
0.183346000 


$\begin{array}{llll}\mathrm{H} & 3.077356000 & -1.556258000 & 0.330072000 \\ \mathrm{H} & 0.738557000 & -2.392856000 & 0.129871000\end{array}$

\section{HCTH/6-311+G(d,p)}

$\begin{array}{lrrr}\mathrm{C} & -0.134870000 & -0.419769000 & -0.126899000 \\ \mathrm{C} & 0.148095000 & 0.979751000 & -0.206396000 \\ \mathrm{C} & 1.449817000 & 1.423847000 & -0.096882000 \\ \mathrm{C} & 2.506075000 & 0.522375000 & 0.095513000 \\ \mathrm{C} & 2.248201000 & -0.852621000 & 0.175896000 \\ \mathrm{C} & 0.957864000 & -1.325404000 & 0.067095000 \\ \mathrm{~N} & -1.330332000 & -1.020046000 & -0.230621000 \\ \mathrm{O} & -2.432174000 & -0.015424000 & -0.418206000 \\ \mathrm{O} & -2.825671000 & 0.520816000 & 0.674568000 \\ \mathrm{H} & -0.658519000 & 1.685387000 & -0.356914000 \\ \mathrm{H} & 1.656826000 & 2.487874000 & -0.162997000 \\ \mathrm{H} & 3.523724000 & 0.890678000 & 0.181062000 \\ \mathrm{H} & 3.066538000 & -1.550479000 & 0.324900000 \\ \mathrm{H} & 0.735418000 & -2.385345000 & 0.127433000 \\ \text { ts (cis-cis) - } \mathbf{C}_{\mathbf{6}} \mathbf{H}_{\mathbf{5}} \mathrm{NOO} & & \end{array}$

\section{M06-L/6-311+G(d,p)}
$\begin{array}{llll}\text { C } & -0.217277000 & 0.000133000 & -0.462736000\end{array}$
$\begin{array}{llll}\text { C } & 0.440643000 & 1.213534000 & -0.278145000\end{array}$
$\begin{array}{llll}\text { C } & 1.772735000 & 1.204486000 & 0.114184000\end{array}$
$\begin{array}{llll}\text { C } & 2.439472000 & -0.000131000 & 0.311693000\end{array}$
$\begin{array}{llll}\text { C } & 1.772556000 & -1.204619000 & 0.113987000\end{array}$
C $\quad 0.440462000 \quad-1.213398000 \quad-0.278320000$
$\begin{array}{llll}\mathrm{N} & -1.553908000 & 0.000214000 & -0.961314000\end{array}$
$\begin{array}{llll}\text { O } & -2.457659000 & -0.000009000 & -0.048762000\end{array}$
$\begin{array}{llll}\text { O } & -2.153683000 & -0.000154000 & 1.216625000\end{array}$
$\mathrm{H} \quad-0.092686000 \quad 2.144076000 \quad-0.438841000$
$\begin{array}{llll}\mathrm{H} & 2.290845000 & 2.146157000 & 0.263015000\end{array}$ 


$\begin{array}{lrrr}\mathrm{H} & 3.480858000 & -0.000225000 & 0.614653000 \\ \mathrm{H} & 2.290532000 & -2.146390000 & 0.262650000 \\ \mathrm{H} & -0.093010000 & -2.143834000 & -0.439165000 \\ \mathbf{m P W P W} \mathbf{m} / \mathbf{6}-\mathbf{3 1 1 + G}(\mathbf{d}, \mathbf{p}) & & \\ \mathrm{C} & 0.216143000 & 0.000002000 & -0.455859000 \\ \mathrm{C} & -0.449860000 & -1.221123000 & -0.276516000 \\ \mathrm{C} & -1.790288000 & -1.211589000 & 0.117297000 \\ \mathrm{C} & -2.461641000 & -0.000002000 & 0.315940000 \\ \mathrm{C} & -1.790291000 & 1.211587000 & 0.117303000 \\ \mathrm{C} & -0.449863000 & 1.221126000 & -0.276511000 \\ \mathrm{~N} & 1.551735000 & 0.000006000 & -0.971903000 \\ \mathrm{O} & 2.482620000 & 0.000001000 & -0.063153000 \\ \mathrm{O} & 2.200087000 & -0.000006000 & 1.224813000 \\ \mathrm{H} & 0.081756000 & -2.157702000 & -0.442702000 \\ \mathrm{H} & -2.311703000 & -2.158310000 & 0.263329000 \\ \mathrm{H} & -3.509088000 & -0.000004000 & 0.618849000 \\ \mathrm{H} & -2.311708000 & 2.158305000 & 0.263338000 \\ \mathrm{H} & 0.081751000 & 2.157706000 & -0.442693000\end{array}$

\section{OLYP/6-311+G(d,p)}

$\begin{array}{lrrr}\mathrm{C} & 0.210011000 & 0.000000000 & -0.443849000 \\ \mathrm{C} & -0.459560000 & -1.219919000 & -0.267584000 \\ \mathrm{C} & -1.801819000 & -1.210711000 & 0.119231000 \\ \mathrm{C} & -2.474794000 & 0.000000000 & 0.314576000 \\ \mathrm{C} & -1.801819000 & 1.210711000 & 0.119231000 \\ \mathrm{C} & -0.459560000 & 1.219919000 & -0.267584000 \\ \mathrm{~N} & 1.544875000 & 0.000000000 & -0.964961000 \\ \mathrm{O} & 2.490277000 & 0.000000000 & -0.078242000 \\ \mathrm{O} & 2.252124000 & 0.000000000 & 1.207664000 \\ \mathrm{H} & 0.068732000 & -2.156035000 & -0.430625000 \\ \mathrm{H} & -2.321998000 & -2.155976000 & 0.262396000\end{array}$




$\begin{array}{llll}\mathrm{H} & -3.521547000 & 0.000000000 & 0.611681000 \\ \mathrm{H} & -2.321999000 & 2.155976000 & 0.262397000 \\ \mathrm{H} & 0.068732000 & 2.156035000 & -0.430625000\end{array}$

HCTH/6-311+G(d,p)
$\begin{array}{llll}\text { C } & 0.210555000 & 0.000000000 & -0.443774000\end{array}$
C $\quad-0.455782000 \quad-1.214540000 \quad-0.266739000$
C $\quad-1.792537000 \quad-1.205625000 \quad 0.118027000$
$\begin{array}{llll}\text { C } & -2.462715000 & 0.000000000 & 0.312437000\end{array}$
$\begin{array}{llll}\text { C } & -1.792537000 & 1.205625000 & 0.118027000\end{array}$
C $\quad-0.455782000 \quad 1.214540000 \quad-0.266739000$
$\mathrm{N} \quad \begin{array}{llll}1.540433000 & 0.000000000 & -0.954837000\end{array}$
$\begin{array}{llll}\text { O } & 2.474565000 & 0.000000000 & -0.074759000\end{array}$
$\begin{array}{llll}\text { O } & 2.237411000 & 0.000000000 & 1.197630000\end{array}$
$\mathrm{H} \quad 0.071866000 \quad-2.148535000 \quad-0.428780000$
$\mathrm{H} \quad-2.311307000 \quad-2.148793000 \quad 0.261229000$
H $\quad-3.507168000 \quad 0.000000000 \quad 0.608564000$
$\mathrm{H} \quad-2.311307000 \quad 2.148793000 \quad 0.261229000$
$\begin{array}{llll}\mathrm{H} & 0.071866000 & 2.148535000 & -0.428780000\end{array}$
ts (trans-trans) $-\mathrm{C}_{6} \mathrm{H}_{5} \mathrm{NOO}$

M06-L/6-311+G(d,p)
$\begin{array}{llll}\mathrm{N} & -1.455291000 & -0.000001000 & 0.679331000\end{array}$
$\begin{array}{llll}\text { O } & -2.153775000 & 0.000000000 & -0.408880000\end{array}$
$\begin{array}{llll}\text { O } & -3.433844000 & 0.000000000 & -0.285737000\end{array}$
$\begin{array}{llll}\text { C } & -0.076303000 & 0.000000000 & 0.297377000\end{array}$
$\begin{array}{llll}\text { C } & 0.598383000 & 1.212507000 & 0.170483000\end{array}$
$\mathrm{H} \quad 0.054580000 \quad 2.143367000 \quad 0.290579000$
$\begin{array}{llll}\text { C } & 1.960191000 & 1.204607000 & -0.105433000\end{array}$
H $\quad 2.488392000 \quad 2.146125000 \quad-0.214719000$
$\begin{array}{llll}\text { C } & 2.641945000 & 0.000000000 & -0.242988000\end{array}$
H $\quad 3.705288000 \quad 0.000000000 \quad-0.457034000$ 


$\begin{array}{llll}\text { C } & 1.960192000 & -1.204607000 & -0.105434000 \\ \text { H } & 2.488393000 & -2.146125000 & -0.214719000 \\ \text { C } & 0.598383000 & -1.212507000 & 0.170483000 \\ \text { H } & 0.054580000 & -2.143368000 & 0.290578000\end{array}$

\section{mPWPW91/6-311+G(d,p)}
$\begin{array}{llll}\mathrm{N} & -1.458197000 & 0.000000000 & 0.684092000\end{array}$
$\begin{array}{llll}\text { O } & -2.172708000 & 0.000000000 & -0.413090000\end{array}$
$\begin{array}{llll}\text { O } & -3.467479000 & 0.000000000 & -0.282916000\end{array}$
$\begin{array}{llll}\text { C } & -0.075934000 & 0.000000000 & 0.295194000\end{array}$
$\begin{array}{llll}\text { C } & 0.604479000 & 1.220158000 & 0.170140000\end{array}$
$\begin{array}{llll}\mathrm{H} & 0.060505000 & 2.156643000 & 0.293116000\end{array}$
$\begin{array}{llll}\text { C } & 1.974907000 & 1.211753000 & -0.106070000\end{array}$
$\mathrm{H} \quad 2.505916000 \quad 2.158256000 \quad-0.214019000$
$\begin{array}{llll}\text { C } & 2.661351000 & 0.000000000 & -0.243807000\end{array}$
H $\quad 3.730893000 \quad 0.000000000 \quad-0.455941000$
$\begin{array}{llll}\text { C } & 1.974907000 & -1.211753000 & -0.106070000\end{array}$
H $\quad 2.505916000 \quad-2.158256000 \quad-0.214019000$
$\begin{array}{llll}\text { C } & 0.604480000 & -1.220158000 & 0.170140000\end{array}$
$\begin{array}{llll}\mathrm{H} & 0.060505000 & -2.156643000 & 0.293116000\end{array}$

OLYP/6-311+G(d,p)
N $\quad-1.460408000$
0.000000000
0.678445000
O $\quad-2.179211000$
0.000000000
$-0.409724000$
O $\quad-3.464365000$
0.000000000
$-0.277324000$
C $\quad-0.078113000$
$0.000000000 \quad 0.288390000$
C $\quad 0.605173000$
1.219154000
0.166022000
H $\quad 0.064682000$
2.155137000
0.285783000
C $\quad 1.976569000$
1.210895000
$-0.104103000$
H 2.506254000
$2.155898000 \quad-0.208962000$
C 2.664162000
$0.000000000 \quad-0.238979000$
H $\quad 3.732406000$
$0.000000000 \quad-0.445865000$ 


$\begin{array}{llll}\text { C } & 1.976569000 & -1.210894000 & -0.104103000 \\ \text { H } & 2.506255000 & -2.155898000 & -0.208962000 \\ \text { C } & 0.605173000 & -1.219155000 & 0.166022000 \\ \text { H } & 0.064682000 & -2.155137000 & 0.285783000\end{array}$

\section{HCTH/6-311+G(d,p)}
$\begin{array}{llll}\mathrm{N} & 0.375519000 & 1.557216000 & 0.000000000\end{array}$
$\begin{array}{llll}\text { O } & -0.817607000 & 2.049375000 & 0.000000000\end{array}$
$\begin{array}{llll}\text { O } & -0.937649000 & 3.322229000 & 0.000000000\end{array}$
$\begin{array}{llll}\text { C } & 0.268115000 & 0.133905000 & 0.000000000\end{array}$
C $\quad 0.279227000 \quad-0.557350000 \quad-1.213752000$
$\mathrm{H} \quad 0.291890000 \quad-0.004592000 \quad-2.147462000$
C $\quad 0.279227000 \quad-1.949141000 \quad-1.205816000$
$\mathrm{H} \quad 0.278471000 \quad-2.487895000 \quad-2.148666000$
$\begin{array}{llll}\text { C } & 0.279831000 & -2.646895000 & 0.000000000\end{array}$
$\begin{array}{llll}\mathrm{H} & 0.283582000 & -3.732537000 & 0.000000000\end{array}$
C $\quad 0.279227000 \quad-1.949141000 \quad 1.205816000$
$\mathrm{H} \quad 0.278471000 \quad-2.487895000 \quad 2.148666000$
C $\quad 0.279227000 \quad-0.557350000 \quad 1.213752000$
H $\quad 0.291890000 \quad-0.004592000 \quad 2.147462000$

S5. XYZ coordinates of the 4- $\mathrm{RC}_{6} \mathrm{H}_{4} \mathrm{NOO}$ stationary points; gas phase; M06-L/6$311+G(d, p)$
cis-4- $\mathrm{NMe}_{2} \mathrm{C}_{6} \mathrm{H}_{4} \mathrm{NOO}$
$\begin{array}{llll}\text { C } & -1.477417000 & -0.443385000 & 0.000129000\end{array}$
$\begin{array}{llll}\text { C } & -0.934742000 & 0.873043000 & 0.000102000\end{array}$
C $\quad 0.429063000 \quad 1.057718000 \quad-0.000032000$
C $\quad 1.336174000 \quad-0.028696000 \quad-0.000161000$
C $\quad 0.794989000 \quad-1.340483000 \quad-0.000100000$
$\begin{array}{llll}\text { C } & -0.560317000 & -1.528526000 & 0.000034000\end{array}$
$\mathrm{N} \quad-2.765500000 \quad-0.898163000 \quad 0.000235000$ 


\begin{tabular}{|c|c|c|c|}
\hline $\mathrm{O}$ & -3.778883000 & -0.092017000 & 0.000276000 \\
\hline $\mathrm{O}$ & -3.619723000 & 1.232534000 & 0.000080000 \\
\hline $\mathrm{H}$ & -1.603083000 & 1.718092000 & 0.000188000 \\
\hline $\mathrm{H}$ & 0.807411000 & 2.072633000 & -0.000045000 \\
\hline $\mathrm{H}$ & 1.448857000 & -2.203177000 & -0.000131000 \\
\hline $\mathrm{H}$ & -0.975618000 & -2.530805000 & 0.000087000 \\
\hline $\mathrm{N}$ & 2.684354000 & 0.176006000 & -0.000340000 \\
\hline $\mathrm{C}$ & 3.595776000 & -0.949982000 & -0.000316000 \\
\hline $\mathrm{H}$ & 3.462590000 & -1.579150000 & 0.886842000 \\
\hline $\mathrm{H}$ & 3.461929000 & -1.579620000 & -0.887025000 \\
\hline $\mathrm{H}$ & 4.619316000 & -0.580882000 & -0.000818000 \\
\hline $\mathrm{C}$ & 3.219868000 & 1.522400000 & 0.000062000 \\
\hline $\mathrm{H}$ & 4.306891000 & 1.474333000 & -0.000069000 \\
\hline $\mathrm{H}$ & 2.904025000 & 2.083787000 & -0.886176000 \\
\hline $\mathrm{H}$ & 2.904194000 & 2.083219000 & 0.886727000 \\
\hline \multicolumn{4}{|c|}{ trans-4-NMe $\mathrm{C}_{6} \mathrm{H}_{4} \mathrm{NOO}$} \\
\hline $\mathrm{C}$ & -1.385918000 & 0.326161000 & 0.000044000 \\
\hline $\mathrm{C}$ & -0.801828000 & -0.961063000 & -0.000378000 \\
\hline $\mathrm{C}$ & 0.563175000 & -1.107745000 & -0.001181000 \\
\hline $\mathrm{C}$ & 1.426780000 & 0.018622000 & -0.001986000 \\
\hline $\mathrm{C}$ & 0.835842000 & 1.304493000 & -0.000610000 \\
\hline $\mathrm{C}$ & -0.531891000 & 1.446430000 & 0.000191000 \\
\hline $\mathrm{N}$ & -2.725442000 & 0.625104000 & 0.000607000 \\
\hline $\mathrm{O}$ & -3.440854000 & -0.468289000 & 0.000121000 \\
\hline $\mathrm{O}$ & -4.741575000 & -0.296837000 & 0.000625000 \\
\hline $\mathrm{H}$ & -1.437388000 & -1.838395000 & 0.000179000 \\
\hline $\mathrm{H}$ & 0.980217000 & -2.107435000 & -0.000968000 \\
\hline $\mathrm{H}$ & 1.457290000 & 2.190733000 & 0.000034000 \\
\hline $\mathrm{H}$ & -0.985195000 & 2.432247000 & 0.001219000 \\
\hline $\mathrm{N}$ & 2.784535000 & -0.138876000 & -0.004393000 \\
\hline
\end{tabular}




\begin{tabular}{|c|c|c|c|}
\hline $\mathrm{C}$ & 3.650325000 & 1.020879000 & 0.001691000 \\
\hline $\mathrm{H}$ & 3.488815000 & 1.650962000 & -0.880350000 \\
\hline $\mathrm{H}$ & 3.494043000 & 1.639431000 & 0.893055000 \\
\hline $\mathrm{H}$ & 4.687804000 & 0.693227000 & -0.003850000 \\
\hline $\mathrm{C}$ & 3.369119000 & -1.463363000 & 0.002260000 \\
\hline $\mathrm{H}$ & 4.453624000 & -1.375106000 & -0.003194000 \\
\hline $\mathrm{H}$ & 3.080177000 & -2.031978000 & 0.893725000 \\
\hline $\mathrm{H}$ & 3.072769000 & -2.042766000 & -0.879489000 \\
\hline \multicolumn{4}{|c|}{ ts (trans-cis) - 4- $\mathrm{NMe}_{2} \mathrm{C}_{6} \mathrm{H}_{4} \mathrm{NOO}$} \\
\hline C & -1.463535000 & 0.522249000 & -0.150500000 \\
\hline 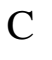 & -0.940980000 & -0.803789000 & -0.223515000 \\
\hline C & 0.410046000 & -1.032176000 & -0.163679000 \\
\hline$c$ & 1.338102000 & 0.030413000 & -0.024292000 \\
\hline $\mathrm{C}$ & 0.823762000 & 1.352180000 & 0.045603000 \\
\hline $\mathrm{C}$ & -0.522571000 & 1.588154000 & -0.013941000 \\
\hline$\Gamma$ & -2.748200000 & 0.891116000 & -0.186730000 \\
\hline $\mathrm{O}$ & -3.594791000 & -0.300266000 & -0.369379000 \\
\hline $\mathrm{O}$ & -3.856955000 & -0.891240000 & 0.755327000 \\
\hline $\mathrm{F}$ & -1.622261000 & -1.639283000 & -0.321105000 \\
\hline $\mathbf{H}$ & 0.766876000 & -2.053505000 & -0.220759000 \\
\hline $\mathrm{H}$ & 1.499912000 & 2.192055000 & 0.149629000 \\
\hline $\mathrm{H}$ & -0.910224000 & 2.599540000 & 0.044056000 \\
\hline $\mathrm{N}$ & 2.684021000 & -0.209427000 & 0.045148000 \\
\hline . & 3.615155000 & 0.888345000 & 0.195866000 \\
\hline H & 3.566256000 & 1.583371000 & -0.650506000 \\
\hline 11 & 3.423725000 & 1.457378000 & 1.112617000 \\
\hline $\mathrm{H}$ & 4.627880000 & 0.493772000 & 0.249855000 \\
\hline $\mathrm{C}$ & 3.190816000 & -1.562503000 & -0.042785000 \\
\hline $\mathrm{H}$ & 4.276220000 & -1.543340000 & 0.031374000 \\
\hline $\mathrm{H}$ & 2.806369000 & -2.192785000 & 0.767196000 \\
\hline
\end{tabular}




\begin{tabular}{|c|c|c|c|}
\hline $\mathrm{H}$ & 2.923691000 & -2.034220000 & -0.995401000 \\
\hline \multicolumn{4}{|c|}{ ts (cis-cis) - 4-NMe ${ }_{2} \mathrm{C}_{6} \mathrm{H}_{4} \mathrm{NOO}$} \\
\hline $\mathrm{C}$ & 1.495650000 & 0.000001000 & -0.511873000 \\
\hline $\mathrm{C}$ & 0.804382000 & -1.203878000 & -0.405861000 \\
\hline $\mathrm{C}$ & -0.559382000 & -1.205993000 & -0.171256000 \\
\hline $\mathrm{C}$ & -1.275947000 & 0.000000000 & -0.029919000 \\
\hline $\mathrm{C}$ & -0.559382000 & 1.205994000 & -0.171252000 \\
\hline $\mathrm{C}$ & 0.804382000 & 1.203880000 & -0.405857000 \\
\hline $\mathrm{N}$ & 2.879033000 & 0.000001000 & -0.847889000 \\
\hline $\mathrm{O}$ & 3.674394000 & 0.000000000 & 0.165787000 \\
\hline $\mathrm{O}$ & 3.231533000 & -0.000002000 & 1.390190000 \\
\hline $\mathrm{H}$ & 1.340136000 & -2.141525000 & -0.509454000 \\
\hline $\mathrm{H}$ & -1.070879000 & -2.157565000 & -0.098847000 \\
\hline $\mathrm{H}$ & -1.070879000 & 2.157566000 & -0.098840000 \\
\hline $\mathrm{H}$ & 1.340136000 & 2.141526000 & -0.509447000 \\
\hline $\mathrm{N}$ & -2.628644000 & 0.000000000 & 0.239091000 \\
\hline $\mathrm{C}$ & -3.363956000 & -1.242141000 & 0.180125000 \\
\hline $\mathrm{H}$ & -3.337883000 & -1.706678000 & -0.816473000 \\
\hline $\mathrm{H}$ & -2.971986000 & -1.968329000 & 0.899193000 \\
\hline $\mathrm{H}$ & -4.404829000 & -1.053860000 & 0.439777000 \\
\hline $\mathrm{C}$ & -3.363957000 & 1.242140000 & 0.180121000 \\
\hline $\mathrm{H}$ & -4.404832000 & 1.053857000 & 0.439764000 \\
\hline $\mathrm{H}$ & -2.971994000 & 1.968328000 & 0.899194000 \\
\hline $\mathrm{H}$ & -3.337876000 & 1.706679000 & -0.816475000 \\
\hline \multicolumn{4}{|c|}{ ts (trans-trans) - 4-NMe $\mathrm{N}_{6} \mathrm{H}_{4} \mathrm{NOO}$} \\
\hline $\mathrm{N}$ & 2.775965000 & 0.000000000 & -0.638507000 \\
\hline $\mathrm{O}$ & 3.407867000 & 0.000000000 & 0.492245000 \\
\hline $\mathrm{O}$ & 4.695194000 & 0.000000000 & 0.446781000 \\
\hline$C$ & 1.377940000 & 0.000000000 & -0.341740000 \\
\hline $\mathrm{C}$ & 0.679720000 & 1.203004000 & -0.264868000 \\
\hline
\end{tabular}




\begin{tabular}{|c|c|c|c|}
\hline I & 1.218029000 & 2.140985000 & -0.353529000 \\
\hline$C$ & -0.692964000 & 1.206160000 & -0.085150000 \\
\hline$\Pi$ & -1.206382000 & 2.157653000 & -0.026665000 \\
\hline $\mathrm{C}$ & -1.415660000 & 0.000000000 & 0.021667000 \\
\hline $\mathrm{C}$ & -0.692964000 & -1.206160000 & -0.085151000 \\
\hline $\mathrm{I}$ & -1.206383000 & -2.157653000 & -0.026667000 \\
\hline $\mathrm{C}$ & 0.679720000 & -1.203003000 & -0.264869000 \\
\hline $\mathrm{H}$ & 1.218028000 & -2.140985000 & -0.353531000 \\
\hline $\mathrm{N}$ & -2.779017000 & 0.000000000 & 0.227935000 \\
\hline $\mathrm{C}$ & -3.510927000 & 1.242246000 & 0.130315000 \\
\hline $\mathrm{H}$ & -3.160664000 & 1.968013000 & 0.871016000 \\
\hline $\mathrm{H}$ & -3.429988000 & 1.707662000 & -0.862706000 \\
\hline $\mathrm{H}$ & -4.564376000 & 1.052611000 & 0.331218000 \\
\hline $\mathrm{C}$ & -3.510927000 & -1.242246000 & 0.130316000 \\
\hline $\mathrm{H}$ & -4.564376000 & -1.052612000 & 0.331223000 \\
\hline $\mathrm{H}$ & -3.429990000 & -1.707662000 & -0.862705000 \\
\hline $\mathrm{H}$ & -3.160661000 & -1.968013000 & 0.871017000 \\
\hline \multicolumn{4}{|c|}{ cis-4-OMeC $6 \mathrm{H}_{4} \mathrm{NOO}$} \\
\hline $\mathrm{C}$ & -1.195288000 & 0.194207000 & 0.000000000 \\
\hline $\mathrm{C}$ & 0.000000000 & 0.958332000 & 0.000000000 \\
\hline $\mathrm{C}$ & 1.228001000 & 0.320545000 & 0.000000000 \\
\hline $\mathrm{C}$ & 1.309076000 & -1.076956000 & 0.000000000 \\
\hline $\mathrm{C}$ & 0.133191000 & -1.848395000 & 0.000000000 \\
\hline $\mathrm{C}$ & -1.084619000 & -1.224047000 & 0.000000000 \\
\hline $\mathrm{N}$ & -2.512776000 & 0.574214000 & 0.000000000 \\
\hline $\mathrm{O}$ & -2.869275000 & 1.816027000 & 0.000000000 \\
\hline $\mathrm{O}$ & -1.982915000 & 2.804650000 & 0.000000000 \\
\hline $\mathrm{H}$ & -0.055842000 & 2.033040000 & 0.000000000 \\
\hline $\mathrm{H}$ & 2.127195000 & 0.924219000 & 0.000000000 \\
\hline $\mathrm{O}$ & 2.458436000 & -1.773168000 & 0.000000000 \\
\hline
\end{tabular}




$\begin{array}{llll}\mathrm{H} & 0.219802000 & -2.928663000 & 0.000000000 \\ \mathrm{H} & -2.004502000 & -1.798068000 & 0.000000000 \\ \mathrm{C} & 3.682147000 & -1.052357000 & 0.000000000 \\ \mathrm{H} & 3.773974000 & -0.427639000 & 0.893715000 \\ \mathrm{H} & 4.469811000 & -1.802799000 & 0.000000000 \\ \mathrm{H} & 3.773974000 & -0.427639000 & -0.893715000\end{array}$

\section{trans-4-OMeC $6 \mathrm{H}_{4} \mathrm{NOO}$}

\begin{tabular}{|c|c|c|c|}
\hline & 1.019614000 & 0.345880000 & .000140000 \\
\hline & 0.352122000 & -0.894467000 & 0.000224000 \\
\hline & -1.027293000 & -0.942630000 & 17000 \\
\hline & -1.773565000 & 0.247428000 & -0.000055000 \\
\hline & -1.116758000 & 1.486066000 & 0.000033000 \\
\hline & 0.256935000 & 35000 & 0.00 \\
\hline & 2.383250000 & 70000 & 0.000044000 \\
\hline & 3.017415000 & -0.588231000 & -0.000098000 \\
\hline & 4.321043000 & -0.514933000 & 9000 \\
\hline & 0.922300000 & -1.815430000 & 4000 \\
\hline & -1.524509000 & -1.905028000 & 231000 \\
\hline & -3.119896000 & 0.299199000 & -0.000333000 \\
\hline & -1.714329000 & 2.389989000 & 0.000016000 \\
\hline & 0.785277000 & 2.479 & 0.000 \\
\hline & -3.839526000 & -0.922946000 & 0.000010000 \\
\hline & -3.618448000 & -1.514844000 & 0.894058000 \\
\hline & -4.892317000 & -0.649392000 & -0.00 \\
\hline & -3.618400000 & -1.515408000 & 58000 \\
\hline \multicolumn{4}{|c|}{ ts (cis-trans) - 4-OMeC $\mathrm{OH}_{4} \mathrm{NOO}$} \\
\hline & -1.111603000 & 0.522858000 & -0.13 \\
\hline & -0.497717000 & -0.758267000 & -0.243538000 \\
\hline & 0.872108000 & -0.888810000 & -0.166437000 \\
\hline & 1.681053000 & 0.242916000 & 0.024 \\
\hline
\end{tabular}




\begin{tabular}{|c|c|c|c|}
\hline $\mathrm{C}$ & 1.092358000 & 1.519912000 & 0.135017000 \\
\hline$C$ & -0.264648000 & 1.659780000 & 0.059878000 \\
\hline I & -2.421106000 & 0.797138000 & -0.181846000 \\
\hline $\mathrm{O}$ & -3.178535000 & -0.450197000 & -0.407321000 \\
\hline $\mathrm{O}$ & -3.410592000 & -1.080066000 & 0.700107000 \\
\hline $\mathrm{I}$ & -1.116054000 & -1.635149000 & -0.387397000 \\
\hline $\mathrm{H}$ & 1.315052000 & -1.873594000 & -0.254233000 \\
\hline $\mathrm{O}$ & 3.024369000 & 0.213484000 & 0.118546000 \\
\hline I & 1.742212000 & 2.375073000 & 0.283586000 \\
\hline I & -0.734884000 & 2.632815000 & 0.147608000 \\
\hline $\mathrm{C}$ & 3.674939000 & -1.043792000 & 0.022072000 \\
\hline H & 3.491151000 & -1.512169000 & -0.950244000 \\
\hline $\mathrm{H}$ & 4.738127000 & -0.839795000 & 0.129821000 \\
\hline $\mathrm{H}$ & 3.351277000 & -1.720488000 & 0.819442000 \\
\hline \multicolumn{4}{|c|}{ ts (cis-cis) - 4-OMeC $6 \mathrm{H}_{4} \mathrm{NOO}$} \\
\hline $\mathrm{C}$ & -1.133567000 & 0.067024000 & -0.509457000 \\
\hline 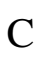 & -0.572240000 & 1.321419000 & -0.263355000 \\
\hline$C$ & 0.782160000 & 1.426948000 & -0.013518000 \\
\hline $\mathrm{C}$ & 1.590735000 & 0.285544000 & -0.011950000 \\
\hline $\mathrm{C}$ & 1.027861000 & -0.965042000 & -0.271229000 \\
\hline $\mathrm{C}$ & -0.335610000 & -1.069478000 & -0.522529000 \\
\hline $\mathrm{N}$ & -2.512562000 & -0.031198000 & -0.856212000 \\
\hline $\mathrm{O}$ & -3.298558000 & -0.220808000 & 0.145366000 \\
\hline $\mathrm{O}$ & -2.850176000 & -0.313088000 & 1.363065000 \\
\hline $\mathrm{H}$ & -1.203228000 & 2.203180000 & -0.264256000 \\
\hline $\mathrm{H}$ & 1.240089000 & 2.389414000 & 0.184899000 \\
\hline $\mathrm{H}$ & 1.635829000 & -1.860922000 & -0.283503000 \\
\hline $\mathrm{H}$ & -0.781042000 & -2.037279000 & -0.725878000 \\
\hline U & 2.905218000 & 0.495584000 & 0.246530000 \\
\hline $\mathrm{C}$ & 3.752926000 & -0.635460000 & 0.283555000 \\
\hline
\end{tabular}




\begin{tabular}{|c|c|c|c|}
\hline $\mathrm{H}$ & 3.785332000 & -1.146283000 & -0.685772000 \\
\hline $\mathrm{H}$ & 4.746346000 & -0.261157000 & 0.524161000 \\
\hline $\mathrm{H}$ & 3.439130000 & -1.347795000 & 1.055041000 \\
\hline \multicolumn{4}{|c|}{ ts (trans-trans) - 4-OMeC $6 \mathrm{H}_{4} \mathrm{NOO}$} \\
\hline $\mathbf{N}$ & -2.397565000 & -0.048765000 & -0.642114000 \\
\hline $\mathrm{O}$ & -3.021933000 & -0.157676000 & 0.486859000 \\
\hline $\mathrm{O}$ & -4.304352000 & -0.252631000 & 0.442176000 \\
\hline $\mathrm{C}$ & -1.002290000 & 0.051359000 & -0.345454000 \\
\hline 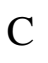 & -0.211895000 & -1.091272000 & -0.324135000 \\
\hline $\mathrm{H}$ & -0.672570000 & -2.064255000 & -0.456685000 \\
\hline $\mathrm{C}$ & 1.162827000 & -0.987432000 & -0.138049000 \\
\hline $\mathrm{H}$ & 1.762024000 & -1.889118000 & -0.116745000 \\
\hline C & 1.747310000 & 0.270744000 & 0.017855000 \\
\hline 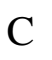 & 0.947765000 & 1.418485000 & -0.013510000 \\
\hline $\mathrm{H}$ & 1.421141000 & 2.385650000 & 0.111804000 \\
\hline C & -0.417210000 & 1.311520000 & -0.199830000 \\
\hline $\mathrm{H}$ & -1.039930000 & 2.198709000 & -0.234550000 \\
\hline $\mathrm{O}$ & 3.073306000 & 0.482493000 & 0.201738000 \\
\hline $\mathrm{C}$ & 3.918566000 & -0.650889000 & 0.236443000 \\
\hline $\mathrm{H}$ & 3.882752000 & -1.207798000 & -0.706894000 \\
\hline $\mathrm{H}$ & 4.926873000 & -0.271313000 & 0.390550000 \\
\hline $\mathrm{H}$ & 3.656060000 & -1.323093000 & 1.061219000 \\
\hline \multicolumn{4}{|c|}{ cis - 4- $\mathrm{MeC}_{6} \mathrm{H}_{4} \mathrm{NOO}$} \\
\hline C & 0.683168000 & 0.412438000 & -0.000471000 \\
\hline $\mathrm{C}$ & 0.228151000 & -0.930035000 & -0.003168000 \\
\hline $\mathrm{C}$ & -1.134392000 & -1.179566000 & -0.005309000 \\
\hline $\mathrm{C}$ & -2.084697000 & -0.154373000 & -0.004040000 \\
\hline $\mathrm{C}$ & -1.627838000 & 1.173490000 & -0.003199000 \\
\hline$C$ & -0.281838000 & 1.452667000 & -0.000979000 \\
\hline $\mathrm{N}$ & 1.955067000 & 0.940401000 & 0.001129000 \\
\hline
\end{tabular}




\begin{tabular}{|c|c|c|c|}
\hline $\mathrm{O}$ & 3.006155000 & 0.192006000 & 0.002569000 \\
\hline $\mathrm{O}$ & 2.935494000 & -1.128708000 & 0.002332000 \\
\hline $\mathrm{H}$ & 0.938178000 & -1.738824000 & -0.004742000 \\
\hline $\mathrm{H}$ & -1.475287000 & -2.211001000 & -0.008810000 \\
\hline $\mathrm{H}$ & -2.347835000 & 1.986962000 & -0.004916000 \\
\hline $\mathrm{H}$ & 0.082224000 & 2.474352000 & -0.000804000 \\
\hline $\mathrm{C}$ & -3.548026000 & -0.449642000 & 0.005661000 \\
\hline $\mathrm{H}$ & -4.005087000 & -0.126270000 & 0.946660000 \\
\hline $\mathrm{H}$ & -4.067329000 & 0.090144000 & -0.791181000 \\
\hline $\mathrm{H}$ & -3.750695000 & -1.514426000 & -0.114280000 \\
\hline \multicolumn{4}{|c|}{ trans - 4-MeC $6 \mathrm{H}_{4} \mathrm{NOO}$} \\
\hline $\mathrm{C}$ & -0.583441000 & 0.297056000 & -0.000024000 \\
\hline $\mathrm{C}$ & -0.056448000 & -1.012016000 & -0.000027000 \\
\hline $\mathrm{C}$ & 1.309359000 & -1.191418000 & 0.000005000 \\
\hline $\mathrm{C}$ & 2.198808000 & -0.101751000 & 0.000027000 \\
\hline $\mathrm{C}$ & 1.663522000 & 1.188944000 & 0.000030000 \\
\hline $\mathrm{C}$ & 0.294903000 & 1.392958000 & -0.000005000 \\
\hline $\mathrm{N}$ & -1.921766000 & 0.647514000 & -0.000056000 \\
\hline $\mathrm{O}$ & -2.666104000 & -0.419072000 & 0.000067000 \\
\hline $\mathrm{O}$ & -3.952052000 & -0.219131000 & -0.000006000 \\
\hline $\mathrm{H}$ & -0.724928000 & -1.864499000 & -0.000054000 \\
\hline $\mathrm{H}$ & 1.713340000 & -2.200400000 & 0.000006000 \\
\hline $\mathrm{H}$ & 2.334093000 & 2.043015000 & 0.000059000 \\
\hline $\mathrm{H}$ & -0.129396000 & 2.391381000 & -0.000006000 \\
\hline $\mathrm{C}$ & 3.673935000 & -0.335426000 & -0.000009000 \\
\hline $\mathrm{H}$ & 3.983213000 & -0.913507000 & 0.876240000 \\
\hline $\mathrm{H}$ & 3.983189000 & -0.913237000 & -0.876446000 \\
\hline $\mathrm{H}$ & 4.234265000 & 0.600198000 & 0.000124000 \\
\hline \multicolumn{4}{|c|}{ ts (cis-trans) - 4-MeC ${ }_{6} \mathrm{H}_{4} \mathrm{NOO}$} \\
\hline $\mathrm{C}$ & -0.659659000 & 0.489635000 & -0.126506000 \\
\hline
\end{tabular}




\begin{tabular}{|c|c|c|c|}
\hline $\mathrm{C}$ & -0.236051000 & -0.867069000 & -0.238769000 \\
\hline $\mathrm{C}$ & 1.103893000 & -1.173320000 & -0.166708000 \\
\hline $\mathrm{C}$ & 2.084544000 & -0.182588000 & 0.016125000 \\
\hline $\mathrm{C}$ & 1.664462000 & 1.158302000 & 0.123403000 \\
\hline $\mathrm{C}$ & 0.336345000 & 1.496792000 & 0.058363000 \\
\hline $\mathrm{N}$ & -1.918945000 & 0.944877000 & -0.170499000 \\
\hline $\mathrm{O}$ & -2.845107000 & -0.184494000 & -0.408160000 \\
\hline $\mathrm{O}$ & -3.172521000 & -0.777597000 & 0.693688000 \\
\hline $\mathrm{H}$ & -0.972723000 & -1.647569000 & -0.377937000 \\
\hline $\mathrm{H}$ & 1.417360000 & -2.210028000 & -0.253989000 \\
\hline $\mathrm{H}$ & 2.410087000 & 1.936266000 & 0.261515000 \\
\hline $\mathrm{H}$ & 0.006309000 & 2.526634000 & 0.142169000 \\
\hline $\mathrm{C}$ & 3.531568000 & -0.532811000 & 0.114775000 \\
\hline $\mathrm{H}$ & 3.887826000 & -0.436024000 & 1.146638000 \\
\hline $\mathrm{H}$ & 3.725752000 & -1.557998000 & -0.203491000 \\
\hline $\mathrm{H}$ & 4.148417000 & 0.137665000 & -0.489720000 \\
\hline \multicolumn{4}{|c|}{ ts (cis-cis) - 4- $\mathrm{MeC}_{6} \mathrm{H}_{4} \mathrm{NOO}$} \\
\hline $\mathrm{C}$ & -0.718975000 & 0.000144000 & -0.502034000 \\
\hline $\mathrm{C}$ & -0.041442000 & 1.209491000 & -0.372394000 \\
\hline $\mathrm{C}$ & 1.316567000 & 1.197268000 & -0.089813000 \\
\hline $\mathrm{C}$ & 2.018181000 & -0.001277000 & 0.062336000 \\
\hline $\mathrm{C}$ & 1.315739000 & -1.198349000 & -0.090270000 \\
\hline $\mathrm{C}$ & -0.042975000 & -1.209316000 & -0.373122000 \\
\hline $\mathrm{N}$ & -2.092076000 & 0.001185000 & -0.887006000 \\
\hline $\mathrm{O}$ & -2.917311000 & 0.000594000 & 0.098155000 \\
\hline $\mathrm{O}$ & -2.510151000 & -0.000701000 & 1.334518000 \\
\hline $\mathrm{H}$ & -0.579667000 & 2.143539000 & -0.492078000 \\
\hline $\mathrm{H}$ & 1.844286000 & 2.141695000 & 0.011862000 \\
\hline $\mathrm{H}$ & 1.842764000 & -2.143125000 & 0.010899000 \\
\hline $\mathrm{H}$ & -0.581803000 & -2.142949000 & -0.493251000 \\
\hline
\end{tabular}




\begin{tabular}{|c|c|c|c|}
\hline $\mathrm{C}$ & 3.476968000 & -0.000039000 & 0.400407000 \\
\hline $\mathrm{H}$ & 3.965307000 & -0.924858000 & 0.087352000 \\
\hline $\mathrm{H}$ & 4.000434000 & 0.835859000 & -0.068607000 \\
\hline $\mathrm{H}$ & 3.628527000 & 0.094872000 & 1.480817000 \\
\hline \multicolumn{4}{|c|}{ ts (trans-trans) - 4- $\mathrm{MeC}_{6} \mathrm{H}_{4} \mathrm{NOO}$} \\
\hline $\mathrm{N}$ & -1.972755000 & 0.000806000 & -0.652119000 \\
\hline $\mathrm{O}$ & -2.623492000 & -0.001044000 & 0.465613000 \\
\hline $\mathrm{O}$ & -3.908332000 & -0.001589000 & 0.397582000 \\
\hline $\mathrm{C}$ & -0.578836000 & 0.001167000 & -0.332021000 \\
\hline $\mathrm{C}$ & 0.107860000 & -1.207638000 & -0.240536000 \\
\hline $\mathrm{H}$ & -0.435241000 & -2.141782000 & -0.337236000 \\
\hline $\mathrm{C}$ & 1.480140000 & -1.197070000 & -0.034917000 \\
\hline $\mathrm{H}$ & 2.012041000 & -2.141559000 & 0.041616000 \\
\hline $\mathrm{C}$ & 2.190484000 & 0.001425000 & 0.074090000 \\
\hline $\mathrm{C}$ & 1.480229000 & 1.198702000 & -0.033600000 \\
\hline $\mathrm{H}$ & 2.012062000 & 2.143002000 & 0.043923000 \\
\hline $\mathrm{C}$ & 0.106890000 & 1.209245000 & -0.239571000 \\
\hline $\mathrm{H}$ & -0.436116000 & 2.143493000 & -0.335588000 \\
\hline $\mathrm{C}$ & 3.667770000 & -0.001438000 & 0.318394000 \\
\hline $\mathrm{H}$ & 4.164433000 & -0.810929000 & -0.220944000 \\
\hline $\mathrm{H}$ & 4.128703000 & 0.940442000 & 0.015368000 \\
\hline $\mathrm{H}$ & 3.890778000 & -0.143605000 & 1.381106000 \\
\hline \multicolumn{4}{|c|}{ cis - 4-BrC ${ }_{6} \mathrm{H}_{4} \mathrm{NOO}$} \\
\hline $\mathrm{C}$ & 0.555489000 & -1.736301000 & 0.000000000 \\
\hline $\mathrm{C}$ & -0.787996000 & -1.281009000 & 0.000000000 \\
\hline $\mathrm{C}$ & -1.046626000 & 0.078957000 & 0.000000000 \\
\hline $\mathrm{C}$ & 0.000000000 & 0.999593000 & 0.000000000 \\
\hline $\mathrm{C}$ & 1.331374000 & 0.574021000 & 0.000000000 \\
\hline $\mathrm{C}$ & 1.598307000 & -0.775807000 & 0.000000000 \\
\hline $\mathrm{N}$ & 1.081636000 & -3.009258000 & 0.000000000 \\
\hline
\end{tabular}




\begin{tabular}{|c|c|c|c|}
\hline $\mathrm{O}$ & 0.329244000 & -4.057831000 & 0.000000000 \\
\hline $\mathrm{O}$ & -0.989465000 & -3.983030000 & 0.000000000 \\
\hline $\mathrm{H}$ & -1.597845000 & -1.990013000 & 0.000000000 \\
\hline $\mathrm{H}$ & -2.072196000 & 0.429778000 & 0.000000000 \\
\hline $\mathrm{Br}$ & -0.379419000 & 2.846786000 & 0.000000000 \\
\hline $\mathrm{H}$ & 2.137096000 & 1.298224000 & 0.000000000 \\
\hline $\mathrm{H}$ & 2.619627000 & -1.140522000 & 0.000000000 \\
\hline \multicolumn{4}{|c|}{ trans - 4-BrC $\mathrm{Br}_{6} \mathrm{H}_{4} \mathrm{NOO}$} \\
\hline $\mathrm{N}$ & 3.040507000 & 0.611968000 & 0.000153000 \\
\hline $\mathrm{O}$ & 3.736573000 & -0.488310000 & 0.000117000 \\
\hline $\mathrm{O}$ & 5.027120000 & -0.346143000 & 0.000149000 \\
\hline $\mathrm{C}$ & 1.688099000 & 0.321678000 & 0.000085000 \\
\hline $\mathrm{C}$ & 0.861648000 & 1.458782000 & 0.000129000 \\
\hline $\mathrm{H}$ & 1.330474000 & 2.436976000 & 0.000212000 \\
\hline $\mathrm{C}$ & -0.514442000 & 1.328849000 & 0.000068000 \\
\hline $\mathrm{H}$ & -1.151201000 & 2.205331000 & 0.000100000 \\
\hline $\mathrm{C}$ & -1.076651000 & 0.053043000 & -0.000037000 \\
\hline $\mathrm{C}$ & -0.268549000 & -1.089895000 & -0.000085000 \\
\hline $\mathrm{H}$ & -0.725834000 & -2.072706000 & -0.000171000 \\
\hline $\mathrm{C}$ & 1.104464000 & -0.962260000 & -0.000025000 \\
\hline $\mathrm{H}$ & 1.731664000 & -1.845215000 & -0.000065000 \\
\hline $\mathrm{Br}$ & -2.952732000 & -0.142678000 & -0.000117000 \\
\hline \multicolumn{4}{|c|}{ ts (cis-trans) - 4-BrC $\mathrm{Br}_{6} \mathrm{NOO}$} \\
\hline $\mathrm{C}$ & 1.757353000 & 0.511099000 & -0.134215000 \\
\hline $\mathrm{C}$ & 1.221068000 & -0.805616000 & -0.266510000 \\
\hline $\mathrm{C}$ & -0.138015000 & -1.005360000 & -0.214769000 \\
\hline $\mathrm{C}$ & -1.003308000 & 0.083113000 & -0.030303000 \\
\hline $\mathrm{C}$ & -0.504192000 & 1.387591000 & 0.102707000 \\
\hline $\mathrm{C}$ & 0.851021000 & 1.599885000 & 0.053328000 \\
\hline $\mathrm{N}$ & 3.048580000 & 0.860353000 & -0.160586000 \\
\hline
\end{tabular}




\begin{tabular}{|c|c|c|c|}
\hline $\mathrm{O}$ & 3.883496000 & -0.341223000 & -0.386025000 \\
\hline $\mathrm{O}$ & 4.148184000 & -0.951053000 & 0.722597000 \\
\hline $\mathrm{H}$ & 1.889014000 & -1.646062000 & -0.404808000 \\
\hline $\mathrm{H}$ & -0.546936000 & -2.004303000 & -0.315018000 \\
\hline $\mathrm{H}$ & -1.188190000 & 2.216216000 & 0.244912000 \\
\hline $\mathrm{H}$ & 1.267472000 & 2.595668000 & 0.156774000 \\
\hline $\mathrm{Br}$ & -2.860526000 & -0.213430000 & 0.048235000 \\
\hline \multicolumn{4}{|c|}{ ts (cis-cis) - 4- $\mathrm{BrC}_{6} \mathrm{H}_{4} \mathrm{NOO}$} \\
\hline $\mathrm{C}$ & 1.786488000 & 0.000000000 & -0.508116000 \\
\hline $\mathrm{C}$ & 1.106743000 & -1.211109000 & -0.413824000 \\
\hline $\mathrm{C}$ & -0.265712000 & -1.210728000 & -0.207939000 \\
\hline $\mathrm{C}$ & -0.943439000 & 0.000000000 & -0.107243000 \\
\hline $\mathrm{C}$ & -0.265712000 & 1.210728000 & -0.207939000 \\
\hline $\mathrm{C}$ & 1.106743000 & 1.211109000 & -0.413824000 \\
\hline $\mathrm{N}$ & 3.175612000 & 0.000000000 & -0.832361000 \\
\hline $\mathrm{O}$ & 3.957316000 & 0.000000000 & 0.187016000 \\
\hline $\mathrm{O}$ & 3.497755000 & 0.000000000 & 1.403099000 \\
\hline $\mathrm{H}$ & 1.651341000 & -2.144928000 & -0.499840000 \\
\hline $\mathrm{H}$ & -0.805676000 & -2.147169000 & -0.130354000 \\
\hline $\mathrm{H}$ & -0.805676000 & 2.147169000 & -0.130354000 \\
\hline $\mathrm{H}$ & 1.651341000 & 2.144928000 & -0.499840000 \\
\hline $\mathrm{Br}$ & -2.820338000 & 0.000000000 & 0.157695000 \\
\hline \multicolumn{4}{|c|}{ ts (trans-trans) - 4-BrC ${ }_{6} \mathrm{H}_{4} \mathrm{NOO}$} \\
\hline $\mathrm{N}$ & 3.085090000 & 0.000000000 & 0.627820000 \\
\hline $\mathrm{O}$ & 3.698714000 & 0.000000000 & -0.512061000 \\
\hline $\mathrm{O}$ & 4.982705000 & 0.000000000 & -0.486297000 \\
\hline $\mathrm{C}$ & 1.681689000 & 0.000000000 & 0.355306000 \\
\hline $\mathrm{C}$ & 0.995157000 & -1.210141000 & 0.286228000 \\
\hline $\mathrm{H}$ & 1.541461000 & -2.144333000 & 0.358836000 \\
\hline $\mathrm{C}$ & -0.384492000 & -1.210752000 & 0.130306000 \\
\hline
\end{tabular}




\begin{tabular}{|c|c|c|c|}
\hline $\mathrm{H}$ & -0.926671000 & -2.147106000 & 0.067878000 \\
\hline $\mathrm{C}$ & -1.066046000 & 0.000000000 & 0.056097000 \\
\hline $\mathrm{C}$ & -0.384492000 & 1.210752000 & 0.130306000 \\
\hline $\mathrm{H}$ & -0.926671000 & 2.147106000 & 0.067878000 \\
\hline $\mathrm{C}$ & 0.995157000 & 1.210141000 & 0.286228000 \\
\hline $\mathrm{H}$ & 1.541461000 & 2.144333000 & 0.358837000 \\
\hline $\mathrm{Br}$ & -2.951383000 & 0.000000000 & -0.135090000 \\
\hline \multicolumn{4}{|c|}{ cis - $4-\mathrm{NO}_{2} \mathrm{C}_{6} \mathrm{H}_{4} \mathrm{NOO}$} \\
\hline $\mathrm{C}$ & -1.346190000 & 0.430407000 & -0.000205000 \\
\hline $\mathrm{C}$ & -0.832230000 & -0.892130000 & -0.000186000 \\
\hline $\mathrm{C}$ & 0.539908000 & -1.079525000 & -0.000061000 \\
\hline $\mathrm{C}$ & 1.387982000 & 0.018931000 & -0.000009000 \\
\hline $\mathrm{C}$ & 0.915986000 & 1.327715000 & -0.000032000 \\
\hline $\mathrm{C}$ & -0.446118000 & 1.525461000 & -0.000113000 \\
\hline $\mathrm{N}$ & -2.647266000 & 0.897486000 & -0.000180000 \\
\hline $\mathrm{O}$ & -3.657231000 & 0.094974000 & -0.000057000 \\
\hline $\mathrm{O}$ & -3.524578000 & -1.210460000 & 0.000611000 \\
\hline $\mathrm{H}$ & -1.501660000 & -1.735061000 & -0.000251000 \\
\hline $\mathrm{H}$ & 0.967516000 & -2.074734000 & -0.000038000 \\
\hline $\mathrm{H}$ & 1.617639000 & 2.151757000 & 0.000027000 \\
\hline $\mathrm{H}$ & -0.867260000 & 2.524402000 & -0.000104000 \\
\hline $\mathrm{N}$ & 2.852842000 & -0.210518000 & 0.000070000 \\
\hline $\mathrm{O}$ & 3.238201000 & -1.369843000 & -0.000022000 \\
\hline $\mathrm{O}$ & 3.572195000 & 0.777792000 & 0.000064000 \\
\hline
\end{tabular}

trans - 4- $\mathrm{NO}_{2} \mathrm{C}_{6} \mathrm{H}_{4} \mathrm{NOO}$

$\begin{array}{lrrr}\text { C } & 1.256894000 & 0.321779000 & 0.000159000 \\ \text { C } & 0.700844000 & -0.975747000 & -0.000456000 \\ \text { C } & -0.669203000 & -1.124928000 & -0.000659000 \\ \text { C } & -1.477857000 & 0.011010000 & -0.000633000 \\ \text { C } & -0.955430000 & 1.298094000 & -0.000029000\end{array}$ 

C $\quad 0.417774000$
1.451312000
0.000239000
$\mathrm{N} \quad 2.605414000$
0.636352000
0.000671000
O 3.316079000
$-0.460707000$
0.000470000
O $\quad 4.597364000$
$-0.300955000$
$-0.000516000$
H 1.346565000
$-1.844727000 \quad-0.000528000$
H $\quad-1.137247000$
$-2.101846000 \quad-0.000978000$
H -1.628534000
2.145880000
0.000118000
H $\quad 0.875747000$
2.434126000
0.000752000
$\mathrm{N} \quad-2.945240000$
$-0.165477000 \quad-0.000190000$
O $\quad-3.632016000$
0.845986000
$-0.000540000$
O $\quad-3.370912000$
$-1.311659000$
0.001279000

\section{ts (cis-trans) - 4- $\mathrm{NO}_{2} \mathrm{C}_{6} \mathrm{H}_{4} \mathrm{NOO}$}

$\begin{array}{lrrr}\mathrm{C} & -1.329365000 & 0.508914000 & -0.144478000 \\ \mathrm{C} & -0.833492000 & -0.828003000 & -0.244222000 \\ \mathrm{C} & 0.518549000 & -1.060781000 & -0.177501000 \\ \mathrm{C} & 1.393708000 & 0.017401000 & -0.015967000 \\ \mathrm{C} & 0.947641000 & 1.337534000 & 0.085434000 \\ \mathrm{C} & -0.401449000 & 1.584472000 & 0.024885000 \\ \mathrm{~N} & -2.610945000 & 0.890940000 & -0.187598000 \\ \mathrm{O} & -3.479801000 & -0.299258000 & -0.385735000 \\ \mathrm{O} & -3.766382000 & -0.857246000 & 0.741199000 \\ \mathrm{H} & -1.525920000 & -1.649885000 & -0.369109000 \\ \mathrm{H} & 0.927454000 & -2.061131000 & -0.248307000 \\ \mathrm{H} & 1.671717000 & 2.132173000 & 0.212156000 \\ \mathrm{H} & -0.798162000 & 2.590181000 & 0.104301000 \\ \mathrm{~N} & 2.842902000 & -0.249346000 & 0.052250000 \\ \mathrm{O} & 3.586486000 & 0.717566000 & 0.146769000 \\ \mathrm{O} & 3.200653000 & -1.418527000 & 0.007703000\end{array}$
ts (cis-cis) - 4- $\mathrm{NO}_{2} \mathrm{C}_{6} \mathrm{H}_{4} \mathrm{NOO}$
$\begin{array}{llll}\text { C } & 1.380680000 & 0.000092000 & -0.511525000\end{array}$ 

$\begin{array}{llll}\text { C } & 0.705081000 & -1.213464000 & -0.410871000\end{array}$
C $\quad-0.665623000 \quad-1.204739000 \quad-0.188271000$
C $\quad-1.352072000 \quad-0.000277000 \quad-0.075497000$
C $\quad-0.665906000 \quad 1.204366000 \quad-0.188014000$
$\begin{array}{llll}\text { C } & 0.704798000 & 1.213468000 & -0.410638000\end{array}$
$\begin{array}{llll}\mathrm{N} & 2.769178000 & 0.000232000 & -0.838996000\end{array}$
$\begin{array}{llll}\text { O } & 3.551686000 & -0.000016000 & 0.179432000\end{array}$
$\begin{array}{llll}\text { O } & 3.091822000 & -0.000229000 & 1.396859000\end{array}$
H $\quad 1.254513000 \quad-2.143879000 \quad-0.503757000$
H $\quad-1.198095000 \quad-2.146530000 \quad-0.105602000$
H $\quad-1.198594000 \quad 2.146017000 \quad-0.105126000$
$\mathrm{H} \quad 1.254007000 \quad 2.144031000 \quad-0.503330000$
$\begin{array}{llll}\mathrm{N} & -2.802662000 & -0.000469000 & 0.162594000\end{array}$
$\begin{array}{llll}\text { O } & -3.355233000 & -1.086268000 & 0.253379000\end{array}$
$\begin{array}{llll}\text { O } & -3.353174000 & 1.087182000 & 0.253022000\end{array}$
ts (trans-trans) - 4- $\mathrm{NO}_{2} \mathrm{C}_{6} \mathrm{H}_{4} \mathrm{NOO}$
$\begin{array}{llll}\mathrm{N} & -2.651228000 & 0.000029000 & 0.630048000\end{array}$
O $\quad-3.272266000 \quad-0.000023000 \quad-0.506526000$
O $\quad-4.553161000 \quad-0.000021000 \quad-0.473112000$
$\begin{array}{llll}\text { C } & -1.250992000 & 0.000017000 & 0.353715000\end{array}$
$\begin{array}{llll}\text { C } & -0.569711000 & 1.215514000 & 0.281191000\end{array}$
$\mathrm{H} \quad-1.120894000 \quad 2.145919000 \quad 0.358005000$
$\begin{array}{llll}\text { C } & 0.806194000 & 1.216071000 & 0.113356000\end{array}$
$\mathrm{H} \quad 1.370838000 \quad 2.137355000 \quad 0.041590000$
C $\quad 1.470135000 \quad 0.000001000 \quad 0.033945000$
C $\quad 0.806193000 \quad-1.216061000 \quad 0.113469000$
$\mathrm{H} \quad 1.370837000 \quad-2.137352000 \quad 0.041788000$
C $\quad-0.569711000 \quad-1.215488000 \quad 0.281305000$
$\mathrm{H} \quad-1.120895000 \quad-2.145886000 \quad 0.358207000$
N $\quad 2.939300000 \quad-0.000007000 \quad-0.145173000$ 


\begin{tabular}{|c|c|c|c|}
\hline $\mathrm{O}$ & 3.495901000 & 1.086121000 & -0.213685000 \\
\hline $\mathrm{O}$ & 3.495897000 & -1.086140000 & -0.213626000 \\
\hline \multicolumn{4}{|c|}{ cis - 4- $\mathrm{NH}_{2} \mathrm{C}_{6} \mathrm{H}_{4} \mathrm{NOO}$} \\
\hline $\mathrm{C}$ & 0.667323000 & 0.418380000 & -0.000548000 \\
\hline $\mathrm{C}$ & 0.210898000 & -0.929661000 & -0.002538000 \\
\hline $\mathrm{C}$ & -1.139684000 & -1.196519000 & -0.003648000 \\
\hline $\mathrm{C}$ & -2.097862000 & -0.165923000 & -0.001580000 \\
\hline $\mathrm{C}$ & -1.653499000 & 1.172830000 & -0.001536000 \\
\hline $\mathrm{C}$ & -0.312355000 & 1.449034000 & -0.000280000 \\
\hline $\mathrm{N}$ & 1.927785000 & 0.949333000 & 0.001799000 \\
\hline $\mathrm{O}$ & 2.986934000 & 0.206178000 & 0.002646000 \\
\hline $\mathrm{O}$ & 2.912879000 & -1.123695000 & 0.002538000 \\
\hline $\mathrm{H}$ & 0.929020000 & -1.731524000 & -0.001717000 \\
\hline $\mathrm{H}$ & -1.477668000 & -2.228885000 & -0.007269000 \\
\hline $\mathrm{H}$ & -2.380949000 & 1.979073000 & -0.004102000 \\
\hline $\mathrm{H}$ & 0.042329000 & 2.473975000 & 0.002007000 \\
\hline $\mathrm{N}$ & -3.431837000 & -0.452890000 & -0.044244000 \\
\hline $\mathrm{H}$ & -4.095609000 & 0.273997000 & 0.167192000 \\
\hline $\mathrm{H}$ & -3.736193000 & -1.390455000 & 0.160307000 \\
\hline
\end{tabular}

trans - 4- $\mathrm{NH}_{2} \mathrm{C}_{6} \mathrm{H}_{4} \mathrm{NOO}$

$\begin{array}{lrrr}\mathrm{C} & 0.566773000 & 0.297207000 & -0.001223000 \\ \mathrm{C} & 0.038494000 & -1.014288000 & -0.002968000 \\ \mathrm{C} & -1.320701000 & -1.210824000 & -0.004145000 \\ \mathrm{C} & -2.213591000 & -0.117562000 & -0.001761000 \\ \mathrm{C} & -1.688307000 & 1.186633000 & -0.001980000 \\ \mathrm{C} & -0.326764000 & 1.386607000 & -0.000900000 \\ \mathrm{~N} & 1.895686000 & 0.649042000 & 0.002383000 \\ \mathrm{O} & 2.650508000 & -0.415228000 & 0.000624000 \\ \mathrm{O} & 3.941983000 & -0.198598000 & 0.004921000 \\ \mathrm{H} & 0.710427000 & -1.864100000 & -0.001846000\end{array}$




\begin{tabular}{|c|c|c|c|}
\hline $\mathrm{H}$ & -1.722314000 & -2.220266000 & -0.008771000 \\
\hline 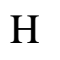 & -2.365626000 & 2.035120000 & -0.004524000 \\
\hline $\mathrm{H}$ & 0.090032000 & 2.388143000 & 0.001785000 \\
\hline $\mathrm{N}$ & -3.566962000 & -0.332035000 & -0.052831000 \\
\hline 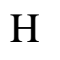 & -4.177073000 & 0.429562000 & 0.197280000 \\
\hline $\mathrm{H}$ & -3.911868000 & -1.243536000 & 0.202720000 \\
\hline \multicolumn{4}{|c|}{ ts (cis-trans) - 4- $\mathrm{NH}_{2} \mathrm{C}_{6} \mathrm{H}_{4} \mathrm{NOO}$} \\
\hline C & -0.642212000 & 0.495709000 & -0.124611000 \\
\hline $\mathrm{C}$ & -0.222039000 & -0.864827000 & -0.231201000 \\
\hline $\mathrm{C}$ & 1.108586000 & -1.189572000 & -0.159030000 \\
\hline $\mathrm{C}$ & 2.094909000 & -0.197732000 & 0.024051000 \\
\hline $\mathrm{C}$ & 1.691522000 & 1.152235000 & 0.125054000 \\
\hline $\mathrm{C}$ & 0.367441000 & 1.491018000 & 0.057003000 \\
\hline $\mathrm{N}$ & -1.897277000 & 0.956710000 & -0.165922000 \\
\hline $\mathrm{O}$ & -2.822958000 & -0.164117000 & -0.409115000 \\
\hline $\mathrm{O}$ & -3.149178000 & -0.776455000 & 0.686026000 \\
\hline $\mathrm{H}$ & -0.963400000 & -1.642074000 & -0.362188000 \\
\hline $\mathrm{H}$ & 1.416044000 & -2.228494000 & -0.241717000 \\
\hline $\mathrm{H}$ & 2.445117000 & 1.922579000 & 0.260188000 \\
\hline $\mathrm{H}$ & 0.049268000 & 2.524451000 & 0.140657000 \\
\hline $\mathrm{N}$ & 3.425186000 & -0.531100000 & 0.049942000 \\
\hline $\mathrm{H}$ & 4.075555000 & 0.140383000 & 0.425861000 \\
\hline $\mathrm{H}$ & 3.669902000 & -1.492521000 & 0.226173000 \\
\hline
\end{tabular}

S6. XYZ coordinates of the $2-\mathrm{RC}_{6} \mathrm{H}_{4} \mathrm{NOO}$ stationary points; gas phase; M06-L/6$311+G(d, p)$

cis /syn - 2-NMe $\mathrm{C}_{6} \mathrm{H}_{4} \mathrm{NOO}$
C $\quad 0.553783000 \quad-0.831760000 \quad 0.243589000$
C $\quad 0.166017000 \quad 0.534421000 \quad 0.032773000$
C $\quad 1.180753000 \quad 1.447884000 \quad-0.328520000$ 


$\begin{array}{lrrr}\mathrm{C} & 2.507945000 & 1.079447000 & -0.280317000 \\ \mathrm{C} & 2.893410000 & -0.207098000 & 0.133179000 \\ \mathrm{C} & 1.924181000 & -1.141767000 & 0.403629000 \\ \mathrm{~N} & -0.273532000 & -1.923330000 & 0.321985000 \\ \mathrm{O} & -1.341161000 & -1.932991000 & -0.448933000 \\ \mathrm{O} & -1.529727000 & -0.969962000 & -1.344513000 \\ \mathrm{H} & 0.919187000 & 2.468393000 & -0.580427000 \\ \mathrm{H} & 3.267043000 & 1.814868000 & -0.527895000 \\ \mathrm{H} & 3.942987000 & -0.469222000 & 0.200378000 \\ \mathrm{H} & 2.173650000 & -2.169144000 & 0.646564000 \\ \mathrm{~N} & -1.109526000 & 0.948151000 & 0.264417000 \\ \mathrm{C} & -1.983509000 & 0.279935000 & 1.210772000 \\ \mathrm{H} & -2.751240000 & -0.319606000 & 0.712790000 \\ \mathrm{H} & -1.404188000 & -0.374325000 & 1.862413000 \\ \mathrm{H} & -2.472726000 & 1.036788000 & 1.830127000 \\ \mathrm{C} & -1.564371000 & 2.233028000 & -0.220979000 \\ \mathrm{H} & -2.653376000 & 2.222524000 & -0.267994000 \\ \mathrm{H} & -1.254053000 & 3.061384000 & 0.430632000 \\ \mathrm{H} & -1.188019000 & 2.413682000 & -1.228599000\end{array}$

trans /syn - 2-NMe $\mathrm{C}_{6} \mathrm{H}_{4} \mathrm{NOO}$
$\begin{array}{llll}\text { C } & 0.274415000 & -0.898489000 & 0.131693000\end{array}$
C $\quad 0.482518000 \quad 0.518356000 \quad-0.019141000$
$\begin{array}{llll}\text { C } & 1.804536000 & 0.952271000 & -0.227005000\end{array}$
$\begin{array}{llll}\text { C } & 2.877486000 & 0.082233000 & -0.155427000\end{array}$
$\begin{array}{llll}\text { C } & 2.683984000 & -1.274834000 & 0.123789000\end{array}$
$\begin{array}{llll}\text { C } & 1.400355000 & -1.748089000 & 0.256943000\end{array}$
$\begin{array}{llll}\mathrm{N} & -0.882165000 & -1.631670000 & 0.019417000\end{array}$
$\begin{array}{llll}\text { O } & -1.930776000 & -0.904730000 & -0.265257000\end{array}$
$\begin{array}{llll}\text { O } & -3.029820000 & -1.583118000 & -0.506322000\end{array}$
$\begin{array}{llll}\mathrm{H} & 1.992369000 & 2.006751000 & -0.389337000\end{array}$ 


$\begin{array}{lrrr}\mathrm{H} & 3.528839000 & -1.949044000 & 0.201850000 \\ \mathrm{H} & 1.194662000 & -2.803723000 & 0.397885000 \\ \mathrm{H} & -0.560291000 & 1.416297000 & 0.006555000 \\ \mathrm{~N} & -1.559814000 & 1.342727000 & 1.062855000 \\ \mathrm{C} & -2.573599000 & 1.369067000 & 0.655540000 \\ \mathrm{H} & -1.443428000 & 0.431659000 & 1.645614000 \\ \mathrm{H} & -1.428706000 & 2.193050000 & 1.744217000 \\ \mathrm{H} & -0.361925000 & 2.752654000 & -0.509901000 \\ \mathrm{C} & -1.340177000 & 3.210434000 & -0.668490000 \\ \mathrm{H} & 0.204617000 & 3.399047000 & 0.177588000 \\ \mathrm{H} & 0.156321000 & 2.720110000 & -1.469152000 \\ \mathrm{H} & 3.881721000 & 0.472068000 & -0.287728000\end{array}$

cis /anti - 2-NMe $\mathrm{C}_{6} \mathrm{H}_{4} \mathrm{NOO}$

$\begin{array}{lrrr}\mathrm{C} & -0.616763000 & 0.068852000 & -0.003509000 \\ \mathrm{C} & -1.447125000 & 1.218899000 & 0.140239000 \\ \mathrm{C} & -0.899936000 & 2.480903000 & 0.138305000 \\ \mathrm{C} & 0.485755000 & 2.645629000 & 0.027135000 \\ \mathrm{C} & 1.329009000 & 1.556181000 & -0.042924000 \\ \mathrm{C} & 0.824160000 & 0.241418000 & -0.011216000 \\ \mathrm{~N} & -0.981179000 & -1.200270000 & -0.338693000 \\ \mathrm{O} & -2.215730000 & -1.600692000 & -0.350102000 \\ \mathrm{O} & -3.213705000 & -0.840788000 & 0.102565000 \\ \mathrm{H} & -2.511895000 & 1.067913000 & 0.203355000 \\ \mathrm{H} & -1.547124000 & 3.346649000 & 0.222458000 \\ \mathrm{H} & 0.915058000 & 3.643154000 & 0.034483000 \\ \mathrm{H} & 2.399362000 & 1.715416000 & -0.065992000 \\ \mathrm{~N} & 1.684685000 & -0.819718000 & -0.011255000 \\ \mathrm{C} & 1.400204000 & -2.100674000 & 0.619155000 \\ \mathrm{H} & 1.172506000 & -2.880223000 & -0.112406000 \\ \mathrm{H} & 2.278223000 & -2.401887000 & 1.199705000\end{array}$




\begin{tabular}{|c|c|c|c|}
\hline $\mathrm{H}$ & 0.549575000 & -2.016949000 & 1.292704000 \\
\hline$C$ & 3.076369000 & -0.620602000 & -0.360407000 \\
\hline $\mathrm{H}$ & 3.671267000 & -0.228878000 & 0.476894000 \\
\hline $\mathrm{H}$ & 3.502621000 & -1.580107000 & -0.656776000 \\
\hline $\mathrm{H}$ & 3.171296000 & 0.063028000 & -1.205170000 \\
\hline \multicolumn{4}{|c|}{ trans /anti - 2- $\mathrm{NMe}_{2} \mathrm{C}_{6} \mathrm{H}_{4} \mathrm{NOO}$} \\
\hline $\mathrm{C}$ & -0.470618000 & 0.433614000 & 0.036843000 \\
\hline $\mathrm{C}$ & -0.714607000 & 1.829126000 & 0.121812000 \\
\hline $\mathrm{C}$ & 0.311150000 & 2.736173000 & 0.105715000 \\
\hline $\mathrm{C}$ & 1.634995000 & 2.273047000 & 0.006217000 \\
\hline $\mathrm{C}$ & 1.913467000 & 0.927838000 & -0.048474000 \\
\hline $\mathrm{C}$ & 0.887310000 & -0.051475000 & -0.000954000 \\
\hline $\mathrm{H}$ & -1.743056000 & 2.163671000 & 0.171354000 \\
\hline $\mathrm{H}$ & 0.104903000 & 3.799055000 & 0.159624000 \\
\hline $\mathrm{H}$ & 2.456902000 & 2.981744000 & -0.007053000 \\
\hline $\mathrm{H}$ & 2.946532000 & 0.608815000 & -0.081823000 \\
\hline $\mathrm{N}$ & 1.223147000 & -1.372302000 & -0.000287000 \\
\hline $\mathrm{C}$ & 0.358782000 & -2.442707000 & 0.475415000 \\
\hline $\mathrm{H}$ & -0.258531000 & -2.865644000 & -0.321713000 \\
\hline $\mathrm{H}$ & 0.992109000 & -3.227779000 & 0.893059000 \\
\hline $\mathrm{H}$ & -0.314800000 & -2.080642000 & 1.250873000 \\
\hline $\mathrm{C}$ & 2.577511000 & -1.772726000 & -0.339231000 \\
\hline $\mathrm{H}$ & 3.276993000 & -1.635417000 & 0.495424000 \\
\hline $\mathrm{H}$ & 2.570721000 & -2.828515000 & -0.606250000 \\
\hline $\mathrm{H}$ & 2.947016000 & -1.213428000 & -1.200164000 \\
\hline $\mathrm{N}$ & -1.455857000 & -0.503118000 & -0.091283000 \\
\hline $\mathrm{O}$ & -2.643756000 & 0.040898000 & -0.071040000 \\
\hline $\mathrm{O}$ & -3.648464000 & -0.812305000 & -0.211011000 \\
\hline \multicolumn{4}{|c|}{ ts $\left(\right.$ cis/syn - trans/syn) - 2-NMe $\mathrm{C}_{6} \mathrm{C}_{4} \mathrm{NOO}$} \\
\hline $\mathrm{C}$ & 0.509186000 & -0.848936000 & 0.309420000 \\
\hline
\end{tabular}




\begin{tabular}{|c|c|c|c|}
\hline $\mathrm{C}$ & 0.226183000 & 0.548444000 & 0.025262000 \\
\hline $\mathrm{C}$ & 1.298366000 & 1.355300000 & -0.395996000 \\
\hline $\mathrm{C}$ & 2.604156000 & 0.903422000 & -0.359493000 \\
\hline $\mathrm{C}$ & 2.901214000 & -0.388356000 & 0.113594000 \\
\hline $\mathrm{C}$ & 1.881963000 & -1.235443000 & 0.450952000 \\
\hline $\mathrm{N}$ & -0.325269000 & -1.892657000 & 0.375511000 \\
\hline $\mathrm{O}$ & -1.685024000 & -1.558282000 & -0.126180000 \\
\hline $\mathrm{O}$ & -1.735921000 & -1.624476000 & -1.415350000 \\
\hline $\mathrm{H}$ & 1.104794000 & 2.382338000 & -0.681761000 \\
\hline $\mathrm{H}$ & 3.930845000 & -0.722695000 & 0.177124000 \\
\hline $\mathrm{H}$ & 2.067682000 & -2.263671000 & 0.741656000 \\
\hline $\mathrm{N}$ & -1.038290000 & 1.066542000 & 0.161011000 \\
\hline $\mathrm{C}$ & -1.881804000 & 0.713951000 & 1.290802000 \\
\hline $\mathrm{H}$ & -2.888782000 & 0.448203000 & 0.958790000 \\
\hline $\mathrm{H}$ & -1.466006000 & -0.131443000 & 1.834698000 \\
\hline $\mathrm{H}$ & -1.950647000 & 1.567998000 & 1.977684000 \\
\hline $\mathrm{C}$ & -1.358134000 & 2.344029000 & -0.433279000 \\
\hline $\mathrm{H}$ & -2.444243000 & 2.442255000 & -0.480634000 \\
\hline $\mathrm{H}$ & -0.966481000 & 3.193569000 & 0.146254000 \\
\hline $\mathrm{H}$ & -0.967515000 & 2.401798000 & -1.449973000 \\
\hline $\mathrm{H}$ & 3.406051000 & 1.572056000 & -0.654841000 \\
\hline \multicolumn{4}{|c|}{ ts (cis/anti - trans/anti) - 2-NMe $\mathrm{C}_{6} \mathrm{H}_{4} \mathrm{NOO}$} \\
\hline $\mathrm{C}$ & 0.588531000 & 0.016003000 & -0.175587000 \\
\hline $\mathrm{C}$ & 1.433003000 & 1.162648000 & -0.307471000 \\
\hline $\mathrm{C}$ & 0.932469000 & 2.434008000 & -0.215216000 \\
\hline $\mathrm{C}$ & -0.440840000 & 2.637118000 & 0.003776000 \\
\hline $\mathrm{C}$ & -1.301705000 & 1.561327000 & 0.094622000 \\
\hline $\mathrm{C}$ & -0.846811000 & 0.234678000 & -0.024815000 \\
\hline $\mathrm{N}$ & 1.009464000 & -1.249004000 & -0.112630000 \\
\hline $\mathrm{O}$ & 2.470960000 & -1.313251000 & -0.240797000 \\
\hline
\end{tabular}




\begin{tabular}{|c|c|c|c|}
\hline 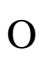 & 3.084758000 & -1.023086000 & 0.864564000 \\
\hline & 2.492569000 & 1.002163000 & -0.452800000 \\
\hline $\mathrm{H}$ & 1.599695000 & 3.284365000 & -0.306309000 \\
\hline I & -2.361874000 & 1.745674000 & 0.214335000 \\
\hline$U$ & -0.838236000 & 3.644137000 & 0.074135000 \\
\hline 1 & -1.735892000 & -0.803909000 & 0.050651000 \\
\hline $\mathrm{C}$ & -3.075679000 & -0.565434000 & 0.544802000 \\
\hline $\mathrm{H}$ & -3.507623000 & -1.516983000 & 0.856543000 \\
\hline I & -3.056340000 & 0.097127000 & 1.411500000 \\
\hline $\mathrm{H}$ & -3.735254000 & -0.125441000 & -0.216510000 \\
\hline C & -1.535339000 & -2.103953000 & -0.572950000 \\
\hline $\mathrm{H}$ & -1.183880000 & -2.852478000 & 0.143163000 \\
\hline $\mathrm{H}$ & -2.490634000 & -2.433028000 & -0.991552000 \\
\hline $\mathrm{F}$ & -0.800948000 & -2.042816000 & -1.371756000 \\
\hline \multicolumn{4}{|c|}{ ts (cis/syn - cis/anti) - 2-NMe ${ }_{2} \mathrm{C}_{6} \mathrm{H}_{4} \mathrm{NOO}$} \\
\hline $\mathrm{C}$ & -0.649153000 & 0.389051000 & -0.437043000 \\
\hline $\mathrm{C}$ & -2.010609000 & 0.122245000 & -0.520381000 \\
\hline $\mathrm{C}$ & -2.500437000 & -1.140698000 & -0.217075000 \\
\hline $\mathrm{C}$ & -1.608825000 & -2.130934000 & 0.172228000 \\
\hline $\mathrm{C}$ & -0.246307000 & -1.873045000 & 0.239793000 \\
\hline $\mathrm{C}$ & 0.281223000 & -0.609473000 & -0.065083000 \\
\hline $\mathrm{N}$ & -0.228433000 & 1.711173000 & -0.760756000 \\
\hline $\mathrm{O}$ & -0.211873000 & 2.518519000 & 0.246275000 \\
\hline $\mathrm{O}$ & -0.534686000 & 2.130647000 & 1.443913000 \\
\hline $\mathrm{H}$ & -2.678173000 & 0.927825000 & -0.808411000 \\
\hline $\mathrm{H}$ & -3.563684000 & -1.341279000 & -0.283136000 \\
\hline $\mathrm{H}$ & -1.970756000 & -3.125724000 & 0.411774000 \\
\hline $\mathrm{H}$ & 0.428710000 & -2.672340000 & 0.520942000 \\
\hline $\mathbf{N}$ & 1.641511000 & -0.316839000 & 0.062758000 \\
\hline $\mathrm{C}$ & 2.311719000 & 0.304741000 & -1.068821000 \\
\hline
\end{tabular}




\begin{tabular}{|c|c|c|c|}
\hline [ & 3.269348000 & 0.712900000 & -0.737812000 \\
\hline $\mathrm{H}$ & 2.503719000 & -0.413594000 & -1.881164000 \\
\hline $\mathrm{H}$ & 1.717678000 & 1.124416000 & -1.473588000 \\
\hline 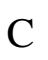 & 2.468064000 & -1.308689000 & 0.716264000 \\
\hline $\mathrm{H}$ & 2.631930000 & -2.213451000 & 0.107569000 \\
\hline $\mathrm{H}$ & 3.444414000 & -0.865195000 & 0.921505000 \\
\hline $\mathrm{H}$ & 2.023693000 & -1.606401000 & 1.667523000 \\
\hline \multicolumn{4}{|c|}{ ts (trans/syn - trans/anti) - 2-NMe $\mathrm{C}_{6} \mathrm{H}_{4} \mathrm{NOO}$} \\
\hline $\mathrm{N}$ & 1.402747000 & -0.871910000 & 0.552531000 \\
\hline $\mathrm{O}$ & 2.014213000 & -0.991508000 & -0.584783000 \\
\hline $\mathrm{O}$ & 3.300576000 & -1.070290000 & -0.544860000 \\
\hline $\mathrm{C}$ & 0.004439000 & -0.792223000 & 0.272942000 \\
\hline $\mathrm{C}$ & -0.619948000 & 0.456643000 & 0.027102000 \\
\hline $\mathrm{C}$ & -2.006317000 & 0.430885000 & -0.193843000 \\
\hline $\mathrm{H}$ & -2.529698000 & 1.362454000 & -0.371913000 \\
\hline $\mathrm{O}$ & -2.727737000 & -0.754987000 & -0.171707000 \\
\hline $\mathrm{H}$ & -3.799272000 & -0.723336000 & -0.341017000 \\
\hline $\mathrm{C}$ & -2.097628000 & -1.966149000 & 0.080403000 \\
\hline $\mathrm{H}$ & -2.659005000 & -2.892944000 & 0.105578000 \\
\hline $\mathrm{C}$ & -0.727182000 & -1.973855000 & 0.304192000 \\
\hline $\mathrm{H}$ & -0.197325000 & -2.899747000 & 0.503230000 \\
\hline $\mathrm{N}$ & 0.123761000 & 1.632861000 & -0.073295000 \\
\hline $\mathrm{C}$ & 1.078747000 & 1.941395000 & 0.977043000 \\
\hline $\mathrm{H}$ & 1.720819000 & 1.083371000 & 1.190831000 \\
\hline $\mathrm{H}$ & 1.712866000 & 2.767405000 & 0.649166000 \\
\hline $\mathrm{H}$ & 0.585555000 & 2.230534000 & 1.917447000 \\
\hline $\mathrm{C}$ & -0.551575000 & 2.803656000 & -0.589644000 \\
\hline $\mathrm{H}$ & -1.284621000 & 3.232667000 & 0.112611000 \\
\hline $\mathrm{H}$ & 0.196219000 & 3.570289000 & -0.800174000 \\
\hline $\mathrm{H}$ & -1.066193000 & 2.564850000 & -1.522197000 \\
\hline
\end{tabular}




\begin{tabular}{lrrr}
\multicolumn{4}{c}{ cis/syn - 2-OMeC $\mathbf{H}_{4} \mathbf{N O O}$} \\
$\mathrm{C}$ & 0.216820000 & -0.844699000 & -0.265278000 \\
$\mathrm{C}$ & 0.097753000 & 0.563656000 & -0.113675000 \\
$\mathrm{C}$ & 1.227950000 & 1.307018000 & 0.233633000 \\
$\mathrm{C}$ & 2.464714000 & 0.686262000 & 0.340626000 \\
$\mathrm{C}$ & 2.608957000 & -0.678385000 & 0.085435000 \\
$\mathrm{C}$ & 1.494916000 & -1.428140000 & -0.235746000 \\
$\mathrm{~N}$ & -0.814362000 & -1.714930000 & -0.574395000 \\
$\mathrm{O}$ & -1.945759000 & -1.554140000 & 0.049748000 \\
$\mathrm{O}$ & -2.075884000 & -0.676507000 & 1.012722000 \\
$\mathrm{O}$ & -1.089387000 & 1.094251000 & -0.428615000 \\
$\mathrm{H}$ & 1.145949000 & 2.375426000 & 0.390826000 \\
$\mathrm{H}$ & 3.332579000 & 1.283314000 & 0.600677000 \\
$\mathrm{H}$ & 3.583720000 & -1.148096000 & 0.148182000 \\
$\mathrm{H}$ & 1.561824000 & -2.495809000 & -0.415073000 \\
$\mathrm{C}$ & -1.277363000 & 2.487391000 & -0.241197000 \\
$\mathrm{H}$ & -0.609644000 & 3.067613000 & -0.886706000 \\
$\mathrm{H}$ & -2.311213000 & 2.685559000 & -0.514703000 \\
$\mathrm{H}$ & -1.116916000 & 2.769054000 & 0.803929000
\end{tabular}

trans/syn - 2-OMeC $\mathrm{OH}_{4} \mathrm{NOO}$
C $\quad-0.020431000$
$-0.793585000$
0.000154000
C $\quad 0.437868000$
0.558318000
0.000356000
C $\quad 1.810120000$
0.807034000
0.000246000
C $\quad 2.722896000$
$-0.241264000 \quad-0.000081000$
C $\quad 2.289845000 \quad-1.565806000 \quad-0.000198000$
$\begin{array}{llll}\text { C } & 0.935923000 & -1.829869000 & -0.000031000\end{array}$
$\begin{array}{llll}\mathrm{N} & -1.311265000 & -1.271218000 & 0.000169000\end{array}$
$\begin{array}{llll}\text { O } & -2.203146000 & -0.320146000 & -0.000963000\end{array}$
$\begin{array}{llll}\text { O } & -3.440941000 & -0.728847000 & 0.000438000\end{array}$
$\begin{array}{llll}\mathrm{H} & 2.171455000 & 1.827497000 & 0.000495000\end{array}$ 


\begin{tabular}{|c|c|c|c|}
\hline I & 3.005214000 & -2.379872000 & -0.000380000 \\
\hline $\mathrm{H}$ & 0.552892000 & -2.844591000 & -0.000120000 \\
\hline $\mathrm{H}$ & 3.784081000 & -0.015125000 & -0.000168000 \\
\hline $\mathrm{O}$ & -0.496529000 & 1.522795000 & 0.000584000 \\
\hline 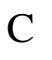 & -0.060412000 & 2.873200000 & -0.000393000 \\
\hline 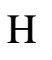 & 0.530796000 & 3.097547000 & -0.894053000 \\
\hline $\mathrm{H}$ & -0.965728000 & 3.475631000 & -0.001129000 \\
\hline 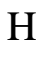 & 0.530210000 & 3.098850000 & 0.893371000 \\
\hline \multicolumn{4}{|c|}{ cis /anti - $2-\mathrm{OMeC}_{6} \mathrm{H}_{4} \mathrm{NOO}$} \\
\hline $\mathrm{C}$ & 0.489771000 & -0.024285000 & 0.000102000 \\
\hline $\mathrm{C}$ & -0.940859000 & -0.194235000 & 0.000130000 \\
\hline $\mathrm{C}$ & -1.774730000 & 0.920184000 & 0.000287000 \\
\hline $\mathrm{C}$ & -1.227738000 & 2.197564000 & 0.000185000 \\
\hline $\mathrm{C}$ & 0.151819000 & 2.384871000 & -0.000090000 \\
\hline $\mathrm{C}$ & 1.006381000 & 1.298774000 & -0.000147000 \\
\hline $\mathrm{N}$ & 1.137820000 & -1.229585000 & 0.000834000 \\
\hline $\mathrm{O}$ & 2.426029000 & -1.320910000 & 0.000518000 \\
\hline $\mathrm{O}$ & 3.222391000 & -0.260600000 & -0.000915000 \\
\hline $\mathrm{O}$ & -1.388048000 & -1.455565000 & -0.000028000 \\
\hline $\mathrm{H}$ & -2.849716000 & 0.794494000 & 0.000533000 \\
\hline $\mathrm{H}$ & -1.892934000 & 3.055433000 & 0.000302000 \\
\hline $\mathrm{H}$ & 0.564847000 & 3.387329000 & -0.000288000 \\
\hline $\mathrm{H}$ & 2.073696000 & 1.431159000 & -0.000333000 \\
\hline $\mathrm{C}$ & -2.789129000 & -1.670182000 & -0.000557000 \\
\hline $\mathrm{H}$ & -3.257461000 & -1.244717000 & -0.894355000 \\
\hline $\mathrm{H}$ & -2.921305000 & -2.749990000 & -0.001454000 \\
\hline $\mathrm{H}$ & -3.257926000 & -1.246164000 & 0.893689000 \\
\hline
\end{tabular}

\section{trans/anti - 2-OMeC $6 \mathrm{H}_{4} \mathrm{NOO}$}
C $\quad-0.401043000$
$0.243237000 \quad-0.000037000$
C $\quad-0.496678000$
$1.649296000-0.000005000$ 


\begin{tabular}{|c|c|c|c|}
\hline $\mathrm{C}$ & 0.638320000 & 2.432880000 & 0.000031000 \\
\hline $\mathrm{C}$ & 1.898827000 & 1.829382000 & 0.000020000 \\
\hline $\mathrm{C}$ & 2.027140000 & 0.448162000 & -0.000029000 \\
\hline $\mathrm{C}$ & 0.890219000 & -0.362792000 & -0.000056000 \\
\hline $\mathrm{H}$ & -1.479583000 & 2.104745000 & -0.000019000 \\
\hline $\mathrm{H}$ & 0.552327000 & 3.513509000 & 0.000047000 \\
\hline $\mathrm{H}$ & 2.793037000 & 2.443838000 & 0.000040000 \\
\hline $\mathrm{H}$ & 3.013450000 & 0.001462000 & -0.000058000 \\
\hline $\mathrm{O}$ & 0.919991000 & -1.702279000 & -0.000099000 \\
\hline $\mathrm{C}$ & 2.183607000 & -2.343628000 & 0.000100000 \\
\hline $\mathrm{H}$ & 2.761642000 & -2.087545000 & -0.894084000 \\
\hline $\mathrm{H}$ & 1.971593000 & -3.410661000 & 0.000232000 \\
\hline $\mathrm{H}$ & 2.761489000 & -2.087287000 & 0.894308000 \\
\hline $\mathrm{N}$ & -1.456675000 & -0.641864000 & -0.000080000 \\
\hline $\mathrm{O}$ & -2.585802000 & 0.008548000 & 0.000074000 \\
\hline $\mathrm{O}$ & -3.661636000 & -0.726799000 & 0.000020000 \\
\hline \multicolumn{4}{|c|}{ ts $(\mathrm{cis} / \mathrm{syn}-\operatorname{trans} / \mathrm{syn})-2-\mathrm{OMeC}_{6} \mathrm{H}_{4} \mathrm{NOO}$} \\
\hline $\mathrm{C}$ & 0.163054000 & -0.879808000 & -0.166312000 \\
\hline $\mathrm{C}$ & 0.227314000 & 0.562857000 & -0.080321000 \\
\hline $\mathrm{C}$ & 1.459733000 & 1.181178000 & 0.108274000 \\
\hline $\mathrm{C}$ & 2.632122000 & 0.436951000 & 0.200119000 \\
\hline $\mathrm{C}$ & 2.598614000 & -0.960153000 & 0.102356000 \\
\hline $\mathrm{C}$ & 1.397331000 & -1.597402000 & -0.075303000 \\
\hline $\mathrm{N}$ & -0.891422000 & -1.689381000 & -0.316478000 \\
\hline $\mathrm{O}$ & -2.174956000 & -0.961571000 & -0.370663000 \\
\hline $\mathrm{O}$ & -2.687865000 & -0.831237000 & 0.805881000 \\
\hline $\mathrm{H}$ & 1.509974000 & 2.260314000 & 0.179917000 \\
\hline $\mathrm{H}$ & 3.514888000 & -1.535297000 & 0.172589000 \\
\hline $\mathrm{H}$ & 1.329972000 & -2.677396000 & -0.144770000 \\
\hline $\mathrm{H}$ & 3.575858000 & 0.951830000 & 0.346710000 \\
\hline
\end{tabular}




\begin{tabular}{|c|c|c|c|}
\hline $\mathrm{O}$ & -0.929008000 & 1.229791000 & -0.201620000 \\
\hline $\mathrm{C}$ & -0.908529000 & 2.642565000 & -0.091109000 \\
\hline $\mathrm{H}$ & -0.525312000 & 2.957525000 & 0.884952000 \\
\hline $\mathrm{H}$ & -1.943834000 & 2.959996000 & -0.194547000 \\
\hline $\mathrm{H}$ & -0.304794000 & 3.095699000 & -0.884511000 \\
\hline \multicolumn{4}{|c|}{ ts $\left(\right.$ cis/anti - trans/anti) - 2-OMeC ${ }_{6} \mathrm{H}_{4} \mathrm{NOO}$} \\
\hline $\mathrm{C}$ & 0.458048000 & -0.041558000 & -0.161654000 \\
\hline $\mathrm{C}$ & 0.973495000 & 1.289503000 & -0.167906000 \\
\hline $\mathrm{C}$ & 0.133605000 & 2.369958000 & -0.047244000 \\
\hline $\mathrm{C}$ & -1.248559000 & 2.182146000 & 0.088047000 \\
\hline $\mathrm{C}$ & -1.796014000 & 0.900936000 & 0.100194000 \\
\hline $\mathrm{C}$ & -0.972803000 & -0.210499000 & -0.022310000 \\
\hline $\mathrm{H}$ & 2.041476000 & 1.429873000 & -0.268149000 \\
\hline $\mathrm{H}$ & 0.543001000 & 3.374240000 & -0.055853000 \\
\hline $\mathrm{H}$ & -1.905864000 & 3.039967000 & 0.184924000 \\
\hline $\mathrm{H}$ & -2.866436000 & 0.775612000 & 0.205737000 \\
\hline $\mathrm{O}$ & -1.397482000 & -1.479732000 & -0.025485000 \\
\hline $\mathrm{C}$ & -2.789069000 & -1.717686000 & 0.099545000 \\
\hline $\mathrm{H}$ & -3.172405000 & -1.339710000 & 1.052926000 \\
\hline $\mathrm{H}$ & -2.909230000 & -2.798156000 & 0.062675000 \\
\hline $\mathrm{H}$ & -3.345462000 & -1.259683000 & -0.724806000 \\
\hline $\mathrm{N}$ & 1.161003000 & -1.170802000 & -0.269190000 \\
\hline $\mathrm{O}$ & 2.593095000 & -0.843210000 & -0.425482000 \\
\hline $\mathrm{O}$ & 3.171348000 & -0.634975000 & 0.712822000 \\
\hline \multicolumn{4}{|c|}{ ts $(\mathrm{cis} / \mathrm{syn}-\mathrm{cis} / \mathrm{anti})-2-\mathrm{OMeC}_{6} \mathrm{H}_{4} \mathrm{NOO}$} \\
\hline $\mathrm{C}$ & -0.445581000 & 0.416852000 & -0.431004000 \\
\hline $\mathrm{C}$ & -0.346414000 & 1.798672000 & -0.353825000 \\
\hline $\mathrm{C}$ & 0.860064000 & 2.385880000 & 0.010116000 \\
\hline $\mathrm{C}$ & 1.955080000 & 1.581208000 & 0.294883000 \\
\hline $\mathrm{C}$ & 1.861912000 & 0.194141000 & 0.222947000 \\
\hline
\end{tabular}




\begin{tabular}{|c|c|c|c|}
\hline $\mathrm{C}$ & 0.656162000 & -0.401486000 & -0.141057000 \\
\hline $\mathrm{N}$ & -1.633471000 & -0.192083000 & -0.928998000 \\
\hline J & -2.534193000 & -0.402360000 & -0.039158000 \\
\hline ) & -2.345030000 & -0.102140000 & 1.213006000 \\
\hline [ & -1.217587000 & 2.401108000 & -0.587292000 \\
\hline $\mathrm{H}$ & 0.939317000 & 3.465430000 & 0.066002000 \\
\hline [ & 2.900625000 & 2.031785000 & 0.578345000 \\
\hline 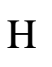 & 2.725732000 & -0.416917000 & 0.454017000 \\
\hline 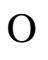 & 0.430744000 & -1.733129000 & -0.226437000 \\
\hline $\mathrm{C}$ & 1.513527000 & -2.596384000 & 0.064875000 \\
\hline . & 2.353377000 & -2.430980000 & -0.619130000 \\
\hline[ & 1.135150000 & -3.608073000 & -0.067392000 \\
\hline $\mathrm{H}$ & 1.857004000 & -2.470023000 & 1.097543000 \\
\hline \multicolumn{4}{|c|}{ ts $(\operatorname{trans} / \mathrm{syn}-\operatorname{trans} / \mathrm{anti})-2-\mathrm{OMeC}_{6} \mathrm{H}_{4} \mathrm{NOO}$} \\
\hline$C$ & 0.278437000 & -0.591379000 & -0.342614000 \\
\hline O & -0.157407000 & -1.903232000 & -0.141319000 \\
\hline 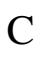 & -1.480902000 & -2.168408000 & 0.168485000 \\
\hline$C$ & -2.382220000 & -1.111540000 & 0.274535000 \\
\hline $\mathrm{C}$ & -1.966399000 & 0.189723000 & 0.051643000 \\
\hline $\mathrm{C}$ & -0.637389000 & 0.474713000 & -0.272605000 \\
\hline U & 0.570790000 & -2.703471000 & -0.222198000 \\
\hline $\mathrm{H}$ & -1.804090000 & -3.190223000 & 0.331698000 \\
\hline $\mathrm{H}$ & -3.421325000 & -1.304007000 & 0.520209000 \\
\hline $\mathrm{H}$ & -2.656719000 & 1.023443000 & 0.116118000 \\
\hline $\mathrm{O}$ & -0.333753000 & 1.782185000 & -0.453054000 \\
\hline $\mathrm{C}$ & -1.060542000 & 2.774070000 & 0.263528000 \\
\hline $\mathrm{H}$ & -2.014809000 & 3.015671000 & -0.214924000 \\
\hline $\mathrm{H}$ & -0.431365000 & 3.664072000 & 0.257014000 \\
\hline П & -1.242126000 & 2.463851000 & 1.296035000 \\
\hline $\mathrm{N}$ & 1.649613000 & -0.381577000 & -0.679436000 \\
\hline
\end{tabular}




\begin{tabular}{|c|c|c|c|}
\hline 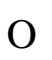 & 2.279556000 & -0.156478000 & 0.437871000 \\
\hline $\mathrm{O}$ & 3.540559000 & 0.089045000 & 0.347955000 \\
\hline \multicolumn{4}{|c|}{ cis/syn - 2-MeC $\mathrm{Me}_{6} \mathrm{H}_{4} \mathrm{NOO}$} \\
\hline $\mathrm{C}$ & 0.041672000 & -0.539954000 & -0.248884000 \\
\hline $\mathrm{C}$ & -0.191615000 & 0.857120000 & -0.161859000 \\
\hline $\mathrm{C}$ & -1.497763000 & 1.260405000 & 0.118188000 \\
\hline $\mathrm{C}$ & -2.531856000 & 0.351934000 & 0.297988000 \\
\hline $\mathrm{C}$ & -2.298882000 & -1.014616000 & 0.145966000 \\
\hline $\mathrm{C}$ & -1.026555000 & -1.452490000 & -0.160737000 \\
\hline $\mathrm{N}$ & 1.247422000 & -1.147333000 & -0.592718000 \\
\hline $\mathrm{O}$ & 2.324999000 & -0.758469000 & 0.008795000 \\
\hline $\mathrm{O}$ & 2.293351000 & 0.108892000 & 0.995893000 \\
\hline $\mathrm{H}$ & -1.702788000 & 2.325748000 & 0.176234000 \\
\hline $\mathrm{H}$ & -3.530530000 & 0.711490000 & 0.524247000 \\
\hline $\mathrm{H}$ & -3.107939000 & -1.728035000 & 0.256473000 \\
\hline $\mathrm{H}$ & -0.807717000 & -2.506360000 & -0.294352000 \\
\hline $\mathrm{C}$ & 0.850058000 & 1.873267000 & -0.473625000 \\
\hline $\mathrm{H}$ & 0.392514000 & 2.815986000 & -0.780370000 \\
\hline $\mathrm{H}$ & 1.487956000 & 2.054489000 & 0.398216000 \\
\hline $\mathrm{H}$ & 1.519386000 & 1.540633000 & -1.271146000 \\
\hline
\end{tabular}

\section{trans /syn - 2-MeC ${ }_{6} \mathrm{H}_{4} \mathrm{NOO}$}
$\begin{array}{llll}\text { C } & 0.008102000 & -0.467507000 & -0.000164000\end{array}$
$\begin{array}{llll}\text { C } & 0.424876000 & 0.895598000 & -0.000156000\end{array}$
$\begin{array}{llll}\text { C } & 1.796893000 & 1.131142000 & 0.000039000\end{array}$
$\begin{array}{llll}\text { C } & 2.736304000 & 0.103789000 & 0.000141000\end{array}$
C $\quad 2.319243000 \quad-1.224551000 \quad 0.000067000$
C $\quad 0.967830000 \quad-1.503164000 \quad-0.000120000$
$\mathrm{N} \quad-1.274948000 \quad-0.983824000 \quad-0.000111000$
O $\quad-2.181318000 \quad-0.050581000 \quad 0.000166000$
$\begin{array}{llll}\text { O } & -3.417832000 & -0.464524000 & 0.000099000\end{array}$ 


\begin{tabular}{|c|c|c|c|}
\hline & 000 & 000 & 00 \\
\hline & .042688000 & .032236000 & .00021900 \\
\hline & 000 & -2.522280000 & -0.00023200 \\
\hline & 000 & 000 & 6000 \\
\hline & 0.512334000 & 2.057973000 & -0.00 \\
\hline & 9000 & 2000 & 000 \\
\hline & 0.047749000 & 94000 & -0.000590000 \\
\hline & -1.173798000 & 2.050 & 5100 \\
\hline \multicolumn{4}{|c|}{ cis /anti - 2- $\mathrm{MeC}_{6} \mathrm{H}_{4} \mathrm{NOO}$} \\
\hline & 000 & 1000 & -0.00 \\
\hline & -0.107619000 & 3000 & -0.0 \\
\hline & 563000 & -1.9 & -0.0 \\
\hline & 2.295006000 & 376000 & 0.00 \\
\hline & 2.1956 & 4000 & 0.0 \\
\hline & 0.964101000 & 0.890462000 & 0.00004800 \\
\hline & -1.345222000 & 5000 & -0. \\
\hline & -2.541855000 & 1000 & -0.00 \\
\hline & -2.793527000 & -0.906774000 & 0.000616000 \\
\hline & -1.000232000 & -1.931934000 & -0.0006420 \\
\hline & 1.224219000 & -3.00 & -0.000430000 \\
\hline & 3.274097000 & 8000 & 0.00 \\
\hline & 3.096499000 & 0.860097000 & 0.000602000 \\
\hline & 0.884011000 & 6000 & 15000 \\
\hline & 0.341486000 & 475000 & 0.87210200 \\
\hline $\mathrm{H}$ & 1.883012000 & 2.820609000 & -0.000351000 \\
\hline & 0.340976000 & .754338000 & -0.8718 \\
\hline
\end{tabular}

trans /anti - 2-MeC ${ }_{6} \mathrm{H}_{4} \mathrm{NOO}$
$\mathrm{N} \quad-1.413440000$
$0.428133000 \quad-0.000026000$
$\begin{array}{llll}\text { O } & -2.270608000 & -0.549676000 & 0.000056000\end{array}$
$\begin{array}{llll}\text { O } & -3.525714000 & -0.205274000 & -0.000055000\end{array}$ 


\begin{tabular}{|c|c|c|c|}
\hline $\mathrm{C}$ & -0.117494000 & -0.055350000 & -0.000017000 \\
\hline $\mathrm{C}$ & 0.874589000 & 0.959848000 & -0.000025000 \\
\hline $\mathrm{C}$ & 2.208677000 & 0.562078000 & -0.000059000 \\
\hline $\mathrm{H}$ & 2.980351000 & 1.326339000 & -0.000131000 \\
\hline $\mathrm{C}$ & 2.566299000 & -0.780880000 & -0.000017000 \\
\hline $\mathrm{H}$ & 3.614739000 & -1.060010000 & -0.000008000 \\
\hline $\mathrm{C}$ & 1.580737000 & -1.771667000 & 0.000033000 \\
\hline $\mathrm{H}$ & 1.861588000 & -2.819203000 & 0.000054000 \\
\hline $\mathrm{C}$ & 0.247463000 & -1.417522000 & 0.000021000 \\
\hline $\mathrm{H}$ & -0.524066000 & -2.178232000 & 0.000038000 \\
\hline $\mathrm{C}$ & 0.499147000 & 2.405690000 & 0.000047000 \\
\hline $\mathrm{H}$ & -0.106956000 & 2.661985000 & 0.872266000 \\
\hline $\mathrm{H}$ & 1.388964000 & 3.036283000 & 0.000446000 \\
\hline $\mathrm{H}$ & -0.106474000 & 2.662322000 & -0.872386000 \\
\hline \multicolumn{4}{|c|}{ ts $(\mathrm{cis} / \mathrm{syn}-\operatorname{trans} / \mathrm{syn})-2-\mathrm{MeC}_{6} \mathrm{H}_{4} \mathrm{NOO}$} \\
\hline $\mathrm{C}$ & 0.009475000 & -0.572991000 & -0.165195000 \\
\hline $\mathrm{C}$ & 0.237577000 & 0.851582000 & -0.097074000 \\
\hline $\mathrm{C}$ & 1.549664000 & 1.269384000 & 0.082605000 \\
\hline $\mathrm{C}$ & 2.624374000 & 0.387551000 & 0.193078000 \\
\hline $\mathrm{C}$ & 2.406766000 & -0.992285000 & 0.114293000 \\
\hline $\mathrm{C}$ & 1.129763000 & -1.461349000 & -0.062005000 \\
\hline $\mathrm{N}$ & -1.132224000 & -1.261568000 & -0.324518000 \\
\hline $\mathrm{O}$ & -2.315926000 & -0.391412000 & -0.371100000 \\
\hline $\mathrm{O}$ & -2.809829000 & -0.207261000 & 0.811588000 \\
\hline $\mathrm{H}$ & 1.738311000 & 2.338164000 & 0.132905000 \\
\hline $\mathrm{H}$ & 3.236353000 & -1.686318000 & 0.193618000 \\
\hline $\mathrm{H}$ & 0.916178000 & -2.523021000 & -0.122168000 \\
\hline $\mathrm{H}$ & 3.627246000 & 0.777060000 & 0.333729000 \\
\hline $\mathrm{C}$ & -0.831412000 & 1.887964000 & -0.231014000 \\
\hline $\mathrm{H}$ & -1.547743000 & 1.850951000 & 0.593661000 \\
\hline
\end{tabular}




\begin{tabular}{|c|c|c|c|}
\hline $\mathrm{H}$ & -0.381093000 & 2.881839000 & -0.246072000 \\
\hline & -1.414883000 & 1.762540000 & -1.146090000 \\
\hline \multicolumn{4}{|c|}{ ts (cis/anti - trans/anti) - $2-\mathrm{MeC}_{6} \mathrm{H}_{4} \mathrm{NOO}$} \\
\hline 4 & -0.194672000 & 0.124839000 & -0.151302000 \\
\hline 4 & -0.122045000 & -1.301192000 & -0.189310000 \\
\hline 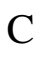 & 1.092704000 & -1.933244000 & -0.066916000 \\
\hline $\mathrm{C}$ & 2.265869000 & -1.185273000 & 0.097172000 \\
\hline 4 & 2.211031000 & 0.209779000 & 0.136855000 \\
\hline 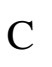 & 1.014870000 & 0.893930000 & 0.017330000 \\
\hline $\mathrm{T}$ & -1.307910000 & 0.859600000 & -0.253624000 \\
\hline $\mathrm{O}$ & -2.482086000 & -0.018191000 & -0.436814000 \\
\hline $\mathrm{O}$ & -2.941450000 & -0.443167000 & 0.695211000 \\
\hline $\mathrm{H}$ & -1.032767000 & -1.872858000 & -0.313487000 \\
\hline $\mathrm{H}$ & 1.141565000 & -3.016537000 & -0.097446000 \\
\hline $\mathrm{H}$ & 3.126882000 & 0.779475000 & 0.266510000 \\
\hline $\mathrm{C}$ & 0.951669000 & 2.382168000 & 0.062504000 \\
\hline $\mathrm{H}$ & 0.520838000 & 2.789637000 & -0.856282000 \\
\hline t & 0.304614000 & 2.726874000 & 0.873733000 \\
\hline $\mathrm{H}$ & 1.944664000 & 2.811410000 & 0.201623000 \\
\hline $\mathrm{H}$ & 3.221305000 & -1.690375000 & 0.195547000 \\
\hline \multicolumn{4}{|c|}{ ts $\left(\right.$ cis/syn - cis/anti) - 2-MeC $6 \mathrm{H}_{4} \mathrm{NOO}$} \\
\hline $\mathrm{C}$ & -0.155367000 & -0.238504000 & -0.424066000 \\
\hline $\mathrm{C}$ & 0.560001000 & -1.432225000 & -0.387676000 \\
\hline $\mathrm{C}$ & 1.896663000 & -1.412164000 & -0.014855000 \\
\hline $\mathrm{C}$ & 2.505174000 & -0.205130000 & 0.309384000 \\
\hline$c$ & 1.776478000 & 0.977354000 & 0.263219000 \\
\hline $\mathrm{C}$ & 0.431628000 & 0.992652000 & -0.103757000 \\
\hline $\mathrm{N}$ & -1.489830000 & -0.255258000 & -0.929034000 \\
\hline $\mathrm{O}$ & -2.396930000 & -0.458847000 & -0.043668000 \\
\hline $\mathrm{O}$ & -2.101956000 & -0.619306000 & 1.215052000 \\
\hline
\end{tabular}




\begin{tabular}{|c|c|c|c|}
\hline $\mathrm{H}$ & 0.064347000 & -2.359630000 & -0.654295000 \\
\hline $\mathrm{H}$ & 2.459917000 & -2.338679000 & 0.015034000 \\
\hline $\mathrm{H}$ & 3.551432000 & -0.183362000 & 0.594975000 \\
\hline $\mathrm{H}$ & 2.255840000 & 1.919683000 & 0.514625000 \\
\hline $\mathrm{C}$ & -0.371636000 & 2.253924000 & -0.131487000 \\
\hline $\mathrm{H}$ & -1.072875000 & 2.293584000 & 0.708593000 \\
\hline $\mathrm{H}$ & 0.270292000 & 3.133292000 & -0.065664000 \\
\hline $\mathrm{H}$ & -0.966702000 & 2.331707000 & -1.045667000 \\
\hline \multicolumn{4}{|c|}{ ts $\left(\operatorname{trans} / \mathrm{syn}\right.$ - trans/anti) - 2- $\mathrm{MeC}_{6} \mathrm{H}_{4} \mathrm{NOO}$} \\
\hline $\mathrm{N}$ & 1.412071000 & -0.312247000 & 0.655965000 \\
\hline $\mathrm{O}$ & 2.115598000 & -0.388389000 & -0.426188000 \\
\hline $\mathrm{O}$ & 3.395861000 & -0.415446000 & -0.295673000 \\
\hline $\mathrm{C}$ & 0.033953000 & -0.284105000 & 0.269853000 \\
\hline $\mathrm{C}$ & -0.588367000 & 0.956908000 & 0.071576000 \\
\hline $\mathrm{C}$ & -1.958100000 & 0.947701000 & -0.190017000 \\
\hline $\mathrm{H}$ & -2.462370000 & 1.896762000 & -0.350236000 \\
\hline $\mathrm{C}$ & -2.679605000 & -0.238937000 & -0.250035000 \\
\hline $\mathrm{H}$ & -3.744728000 & -0.212515000 & -0.454618000 \\
\hline $\mathrm{C}$ & -2.039612000 & -1.455748000 & -0.045885000 \\
\hline $\mathrm{H}$ & -2.596387000 & -2.385553000 & -0.091617000 \\
\hline $\mathrm{C}$ & -0.677133000 & -1.480324000 & 0.222175000 \\
\hline $\mathrm{H}$ & -0.156404000 & -2.415184000 & 0.401014000 \\
\hline $\mathrm{C}$ & 0.199352000 & 2.227997000 & 0.130650000 \\
\hline $\mathrm{H}$ & 0.852503000 & 2.337230000 & -0.741049000 \\
\hline $\mathrm{H}$ & -0.458578000 & 3.097389000 & 0.158505000 \\
\hline $\mathrm{H}$ & 0.846868000 & 2.257324000 & 1.011233000 \\
\hline \multicolumn{4}{|c|}{ cis /syn - 2-BrC $6 \mathrm{H}_{4} \mathrm{NOO}$} \\
\hline $\mathrm{C}$ & 0.775499000 & 0.804312000 & -0.277806000 \\
\hline $\mathrm{C}$ & 0.295858000 & -0.486940000 & 0.032928000 \\
\hline $\mathrm{C}$ & 1.196694000 & -1.494281000 & 0.362995000 \\
\hline
\end{tabular}



$\begin{array}{llll}\text { C } & 2.562177000 & -1.245168000 & 0.363551000\end{array}$
$\begin{array}{llll}\text { C } & 3.049971000 & 0.005231000 & -0.013582000\end{array}$
C $\quad 2.165156000 \quad 1.008406000 \quad-0.356097000$
$\begin{array}{llll}\mathrm{N} & 0.027205000 & 1.919420000 & -0.658371000\end{array}$
$\begin{array}{llll}\text { O } & -0.989346000 & 2.245659000 & 0.079410000\end{array}$
$\begin{array}{llll}\text { O } & -1.285899000 & 1.587707000 & 1.166170000\end{array}$
$\mathrm{Br} \quad-1.512961000 \quad-0.951824000 \quad-0.187559000$
$\mathrm{H} \quad 0.816955000 \quad-2.482103000 \quad 0.598269000$
$\begin{array}{llll}\mathrm{H} & 3.248000000 & -2.042114000 & 0.630988000\end{array}$
$\mathrm{H} \quad 4.117498000 \quad 0.192338000 \quad-0.041817000$
$\mathrm{H} \quad 2.510561000 \quad 1.993478000 \quad-0.650835000$
trans /syn - 2-BrC $\mathrm{C}_{6} \mathrm{H}_{4} \mathrm{NOO}$
$\begin{array}{llll}\text { C } & -0.559433000 & 0.973542000 & 0.000009000\end{array}$
C $\quad-0.518193000 \quad-0.446737000 \quad 0.000011000$
C $\quad-1.710922000 \quad-1.162380000 \quad 0.000069000$
$\begin{array}{llll}\text { C } & -2.940611000 & -0.514974000 & 0.000127000\end{array}$
$\begin{array}{llll}\text { C } & -2.999209000 & 0.876747000 & 0.000127000\end{array}$
$\begin{array}{llll}\text { C } & -1.827018000 & 1.601259000 & 0.000072000\end{array}$
$\begin{array}{llll}\mathrm{N} & 0.447167000 & 1.922602000 & -0.000052000\end{array}$
$\begin{array}{llll}\text { O } & 1.637390000 & 1.402731000 & -0.000114000\end{array}$
$\begin{array}{llll}\text { O } & 2.617813000 & 2.255069000 & 0.000076000\end{array}$
H $\quad-1.667348000 \quad-2.245522000 \quad 0.000069000$
H $\quad-3.954439000 \quad 1.389363000 \quad 0.000167000$
$\mathrm{H} \quad-1.827674000 \quad 2.685878000 \quad 0.000071000$
H $\quad-3.850681000 \quad-1.104967000 \quad 0.000175000$
$\mathrm{Br} \quad 1.070305000 \quad-1.468860000 \quad-0.000066000$
cis /anti - 2-BrC $\mathrm{Br}_{6} \mathrm{NOO}$
$\begin{array}{llll}\text { C } & 0.570758000 & -0.579162000 & 0.000000000 \\ \text { C } & 1.988064000 & -0.683535000 & 0.000000000 \\ \text { C } & 2.772710000 & 0.455059000 & 0.000000000\end{array}$ 


$\begin{array}{cccc}\mathrm{C} & 2.191812000 & 1.719682000 & 0.000000000 \\ \mathrm{C} & 0.807399000 & 1.856251000 & 0.000000000 \\ \mathrm{C} & 0.000000000 & 0.731909000 & 0.000000000 \\ \mathrm{~N} & -0.382316000 & -1.568454000 & 0.000000000 \\ \mathrm{O} & -0.082021000 & -2.822151000 & 0.000000000 \\ \mathrm{O} & 1.161235000 & -3.267295000 & 0.000000000 \\ \mathrm{H} & 2.432534000 & -1.663386000 & 0.000000000 \\ \mathrm{H} & 3.852169000 & 0.352174000 & 0.000000000 \\ \mathrm{H} & 2.814260000 & 2.608393000 & 0.000000000 \\ \mathrm{H} & 0.350824000 & 2.838956000 & 0.000000000 \\ \mathrm{Br} & -1.868335000 & 0.987354000 & 0.000000000\end{array}$

trans /anti - 2-BrC $\mathrm{BH}_{6} \mathrm{NOO}$

$\begin{array}{lrrr}\mathrm{N} & -1.335758000 & -0.855538000 & -0.000007000 \\ \mathrm{O} & -2.631539000 & -0.733309000 & -0.000051000 \\ \mathrm{O} & -3.305950000 & -1.841589000 & -0.000041000 \\ \mathrm{C} & -0.740132000 & 0.391644000 & -0.000016000 \\ \mathrm{C} & 0.675545000 & 0.404054000 & 0.000010000 \\ \mathrm{C} & 1.365124000 & 1.610988000 & 0.000009000 \\ \mathrm{H} & 2.448787000 & 1.602320000 & 0.000028000 \\ \mathrm{C} & 0.667371000 & 2.810326000 & -0.000017000 \\ \mathrm{H} & 1.215758000 & 3.746362000 & -0.000017000 \\ \mathrm{C} & -0.730423000 & 2.814675000 & -0.000042000 \\ \mathrm{H} & -1.271044000 & 3.754773000 & -0.000061000 \\ \mathrm{C} & -1.426948000 & 1.625969000 & -0.000041000 \\ \mathrm{H} & -2.510004000 & 1.623294000 & -0.000057000 \\ \mathrm{Br} & 1.660100000 & -1.202421000 & 0.000042000 \\ \mathrm{ts}(\mathbf{c i s} / \mathbf{s y n}-\mathbf{t r a n s} / \mathbf{s y n})-\mathbf{2 - B r C} \mathbf{H} \mathbf{4} \mathbf{N O O} \\ \mathrm{C} & -0.744290000 & 0.909839000 & -0.210728000 \\ \mathrm{C} & -0.381099000 & -0.465699000 & 0.004142000 \\ \mathrm{C} & -1.374638000 & -1.404463000 & 0.248537000\end{array}$




\begin{tabular}{|c|c|c|c|}
\hline $\mathrm{C}$ & -2.720235000 & -1.052696000 & 0.278051000 \\
\hline $\mathrm{C}$ & -3.104579000 & 0.272779000 & 0.039770000 \\
\hline $\mathrm{C}$ & -2.145663000 & 1.221159000 & -0.202748000 \\
\hline $\mathrm{N}$ & -0.014825000 & 2.012267000 & -0.438610000 \\
\hline $\mathrm{O}$ & 1.433888000 & 1.801149000 & -0.280214000 \\
\hline $\mathrm{O}$ & 1.784499000 & 1.973528000 & 0.951564000 \\
\hline $\mathrm{H}$ & -1.082996000 & -2.436241000 & 0.407897000 \\
\hline $\mathrm{H}$ & -4.152292000 & 0.551009000 & 0.051062000 \\
\hline $\mathrm{H}$ & -2.402380000 & 2.259946000 & -0.379160000 \\
\hline $\mathrm{H}$ & -3.465442000 & -1.815773000 & 0.475189000 \\
\hline $\mathrm{Br}$ & 1.379509000 & -1.135078000 & -0.108505000 \\
\hline \multicolumn{4}{|c|}{ ts (cis/anti - trans/anti) - 2-BrC ${ }_{6} \mathrm{H}_{4} \mathrm{NOO}$} \\
\hline $\mathrm{C}$ & 0.775017000 & 0.020365000 & -0.154257000 \\
\hline $\mathrm{C}$ & 1.831605000 & 0.985382000 & -0.173396000 \\
\hline $\mathrm{C}$ & 1.557998000 & 2.324946000 & -0.051958000 \\
\hline $\mathrm{C}$ & 0.237533000 & 2.774084000 & 0.094465000 \\
\hline $\mathrm{C}$ & -0.818577000 & 1.864881000 & 0.116741000 \\
\hline $\mathrm{C}$ & -0.569914000 & 0.510782000 & -0.004514000 \\
\hline $\mathrm{N}$ & 0.915041000 & -1.304099000 & -0.263780000 \\
\hline $\mathrm{O}$ & 2.338270000 & -1.647331000 & -0.438492000 \\
\hline $\mathrm{O}$ & 2.962129000 & -1.724072000 & 0.692770000 \\
\hline $\mathrm{H}$ & 2.850740000 & 0.640222000 & -0.287236000 \\
\hline $\mathrm{H}$ & 2.371686000 & 3.041694000 & -0.071712000 \\
\hline $\mathrm{H}$ & -1.839613000 & 2.211290000 & 0.227724000 \\
\hline $\mathrm{H}$ & 0.029855000 & 3.834529000 & 0.189182000 \\
\hline $\mathrm{Br}$ & -2.008661000 & -0.700299000 & 0.022623000 \\
\hline \multicolumn{4}{|c|}{ ts (cis/syn - cis/anti) - 2-BrC ${ }_{6} \mathrm{H}_{4} \mathrm{NOO}$} \\
\hline $\mathrm{C}$ & -0.776490000 & 0.513347000 & -0.422744000 \\
\hline $\mathrm{C}$ & -2.168866000 & 0.543253000 & -0.437600000 \\
\hline $\mathrm{C}$ & -2.884802000 & -0.584473000 & -0.062086000 \\
\hline
\end{tabular}




\begin{tabular}{|c|c|c|c|}
\hline $\mathrm{C}$ & -2.212611000 & -1.741892000 & 0.316206000 \\
\hline $\mathrm{C}$ & -0.824026000 & -1.778774000 & 0.320516000 \\
\hline $\mathrm{C}$ & -0.104174000 & -0.650190000 & -0.053332000 \\
\hline $\mathrm{N}$ & -0.059503000 & 1.646199000 & -0.915584000 \\
\hline $\mathrm{O}$ & 0.238042000 & 2.511236000 & -0.014385000 \\
\hline $\mathrm{O}$ & -0.065735000 & 2.326402000 & 1.234499000 \\
\hline $\mathrm{H}$ & -2.672305000 & 1.454932000 & -0.741057000 \\
\hline $\mathrm{H}$ & -3.968789000 & -0.557952000 & -0.069055000 \\
\hline $\mathrm{H}$ & -2.769290000 & -2.625862000 & 0.607640000 \\
\hline $\mathrm{H}$ & -0.292023000 & -2.677147000 & 0.611947000 \\
\hline $\mathrm{Br}$ & 1.787608000 & -0.675031000 & -0.049344000 \\
\hline \multicolumn{4}{|c|}{ ts (trans/syn - trans/anti) - 2-BrC $\mathrm{B}_{6} \mathrm{H}_{4} \mathrm{NOO}$} \\
\hline $\mathrm{N}$ & -0.760502000 & 1.468293000 & 0.646356000 \\
\hline $\mathrm{O}$ & -1.370923000 & 1.831702000 & -0.442683000 \\
\hline $\mathrm{O}$ & -2.508909000 & 2.408799000 & -0.302147000 \\
\hline $\mathrm{C}$ & 0.470440000 & 0.856922000 & 0.257376000 \\
\hline $\mathrm{C}$ & 0.546814000 & -0.516941000 & 0.016146000 \\
\hline $\mathrm{C}$ & 1.777508000 & -1.111180000 & -0.240477000 \\
\hline $\mathrm{H}$ & 1.824155000 & -2.177481000 & -0.429947000 \\
\hline $\mathrm{C}$ & 2.929633000 & -0.334475000 & -0.251821000 \\
\hline $\mathrm{H}$ & 3.886407000 & -0.805355000 & -0.450520000 \\
\hline $\mathrm{C}$ & 2.858766000 & 1.032837000 & -0.007528000 \\
\hline $\mathrm{H}$ & 3.757990000 & 1.638382000 & -0.017363000 \\
\hline $\mathrm{C}$ & 1.632366000 & 1.627241000 & 0.254226000 \\
\hline $\mathrm{H}$ & 1.555287000 & 2.688980000 & 0.463167000 \\
\hline $\mathrm{Br}$ & -1.027281000 & -1.567800000 & 0.048608000 \\
\hline \multicolumn{4}{|c|}{ cis $/ \mathrm{syn}-2-\mathrm{NO}_{2} \mathrm{C}_{6} \mathrm{H}_{4} \mathrm{NOO}$} \\
\hline $\mathrm{C}$ & -0.386599000 & -0.831618000 & -0.222038000 \\
\hline $\mathrm{C}$ & -0.233631000 & 0.556188000 & -0.027053000 \\
\hline $\mathrm{C}$ & -1.326412000 & 1.365069000 & 0.225692000 \\
\hline
\end{tabular}




$\begin{array}{lrrr}\mathrm{C} & -2.604148000 & 0.815640000 & 0.255062000 \\ \mathrm{C} & -2.785692000 & -0.540770000 & 0.003419000 \\ \mathrm{C} & -1.690879000 & -1.351472000 & -0.246282000 \\ \mathrm{~N} & 0.579012000 & -1.817393000 & -0.412981000 \\ \mathrm{O} & 1.668137000 & -1.754666000 & 0.276934000 \\ \mathrm{O} & 1.906592000 & -0.775816000 & 1.104532000 \\ \mathrm{H} & -1.157350000 & 2.425762000 & 0.369944000 \\ \mathrm{H} & -3.457537000 & 1.456194000 & 0.448109000 \\ \mathrm{H} & -3.782317000 & -0.967975000 & 0.000687000 \\ \mathrm{H} & -1.803621000 & -2.415797000 & -0.419761000 \\ \mathrm{~N} & 1.051328000 & 1.226498000 & -0.284001000 \\ \mathrm{O} & 1.272450000 & 2.266308000 & 0.311171000 \\ \mathrm{O} & 1.771899000 & 0.709158000 & -1.124250000\end{array}$

\section{trans /syn - 2- $\mathrm{NO}_{2} \mathrm{C}_{6} \mathrm{H}_{4} \mathrm{NOO}$}
$\begin{array}{llll}\text { C } & 0.259657000 & -0.857228000 & 0.106958000\end{array}$
$\begin{array}{llll}\text { C } & 0.465420000 & 0.535144000 & -0.013852000\end{array}$
C $\quad \begin{array}{llll}\text { C } & 1.737853000 & 1.057236000 & -0.158920000\end{array}$
$\begin{array}{llll}\text { C } & 2.843941000 & 0.213727000 & -0.137497000\end{array}$
C $\quad 2.669336000 \quad-1.156874000 \quad 0.034239000$
C $\quad 1.395290000 \quad-1.684286000 \quad 0.152699000$
N $\quad 1.847833000 \quad 2.130823000 \quad-0.260923000$
$\begin{array}{llll}\text { O } & 3.838677000 & 0.632517000 & -0.238612000\end{array}$
$\begin{array}{llll}\text { O } & 3.528484000 & -1.817218000 & 0.065115000\end{array}$
$\mathrm{H} \quad 1.229701000 \quad-2.751505000 \quad 0.250055000$
H $\quad-0.621239000 \quad 1.524137000 \quad 0.145445000$
$\mathrm{H} \quad-0.632093000 \quad 2.459846000 \quad-0.637778000$
$\mathrm{H} \quad-1.377352000 \quad 1.368269000 \quad 1.089912000$
$\mathrm{N} \quad-0.935696000 \quad-1.556263000 \quad 0.150558000$
O $\quad-1.890897000 \quad-0.823387000 \quad-0.348728000$
$\begin{array}{llll}\text { O } & -3.071549000 & -1.331734000 & -0.327085000\end{array}$ 


\section{cis /anti - 2- $\mathrm{NO}_{2} \mathrm{C}_{6} \mathrm{H}_{4} \mathrm{NOO}$}

$\begin{array}{lrrr}\mathrm{C} & -0.537974000 & 0.091667000 & -0.020038000 \\ \mathrm{C} & -1.227123000 & 1.329219000 & 0.010955000 \\ \mathrm{C} & -0.512682000 & 2.517097000 & 0.058745000 \\ \mathrm{C} & 0.877642000 & 2.526020000 & 0.070838000 \\ \mathrm{C} & 1.583225000 & 1.325460000 & 0.034908000 \\ \mathrm{C} & 0.881054000 & 0.143113000 & 0.011017000 \\ \mathrm{~N} & -1.003140000 & -1.194596000 & -0.194487000 \\ \mathrm{O} & -2.261162000 & -1.465220000 & -0.190093000 \\ \mathrm{O} & -3.184014000 & -0.555415000 & 0.027164000 \\ \mathrm{H} & -2.302767000 & 1.332130000 & -0.017262000 \\ \mathrm{H} & -1.057899000 & 3.454267000 & 0.082142000 \\ \mathrm{H} & 1.419250000 & 3.464963000 & 0.109611000 \\ \mathrm{H} & 2.666611000 & 1.301008000 & 0.034414000 \\ \mathrm{~N} & 1.672220000 & -1.105820000 & 0.047851000 \\ \mathrm{O} & 1.445758000 & -1.880740000 & 0.958035000 \\ \mathrm{O} & 2.525217000 & -1.229238000 & -0.817731000\end{array}$

\section{trans /anti - 2- $\mathrm{NO}_{2} \mathrm{C}_{6} \mathrm{H}_{4} \mathrm{NOO}$}
C $\quad 0.361488000 \quad-0.409737000$
0.040386000
C $\quad 0.409388000 \quad-1.815891000 \quad 0.012648000$
C $\quad-0.755618000 \quad-2.556556000 \quad-0.065247000$
C $\quad-1.999071000 \quad-1.925042000 \quad-0.082390000$
$\begin{array}{llll}\text { C } & -2.072730000 & -0.539019000 & -0.027510000\end{array}$
$\begin{array}{llll}\text { C } & -0.906936000 & 0.203377000 & 0.011632000\end{array}$
H $\quad-0.698009000 \quad-3.639293000 \quad-0.086983000$
H $\quad-2.909773000 \quad-2.511033000 \quad-0.130520000$
H $\quad-3.021588000 \quad-0.015667000 \quad-0.030920000$
$\begin{array}{llll}\mathrm{N} & 1.466616000 & 0.408727000 & 0.232783000\end{array}$
$\begin{array}{llll}\text { O } & 2.540238000 & -0.253116000 & -0.088540000\end{array}$
$\begin{array}{llll}\text { O } & 3.664470000 & 0.329369000 & 0.156185000\end{array}$ 


\begin{tabular}{|c|c|c|c|}
\hline J & -1.036175000 & 1.673045000 & -0.037817000 \\
\hline $\mathrm{O}$ & -0.221147000 & 2.285676000 & -0.706022000 \\
\hline $\mathrm{O}$ & -1.980282000 & 2.157962000 & 0.570231000 \\
\hline I & 1.370927000 & -2.308328000 & 0.091708000 \\
\hline \multicolumn{4}{|c|}{ ts $(\operatorname{cis} / s y n-\operatorname{trans} / s y n)-2-\mathrm{NO}_{2} \mathrm{C}_{6} \mathrm{H}_{4} \mathrm{NOO}$} \\
\hline $\mathrm{C}$ & 0.399913000 & -0.860190000 & 0.250220000 \\
\hline $\mathrm{C}$ & 0.283855000 & 0.547413000 & 0.022953000 \\
\hline $\mathrm{C}$ & 1.383938000 & 1.316401000 & -0.288382000 \\
\hline $\mathrm{C}$ & 2.656715000 & 0.744813000 & -0.313299000 \\
\hline $\mathrm{C}$ & 2.820023000 & -0.614130000 & -0.022659000 \\
\hline $\mathrm{C}$ & 1.724657000 & -1.399244000 & 0.249917000 \\
\hline J & -0.549006000 & -1.795094000 & 0.384514000 \\
\hline $\mathrm{O}$ & -1.898230000 & -1.227696000 & 0.105959000 \\
\hline $\mathrm{O}$ & -2.160692000 & -1.318814000 & -1.152008000 \\
\hline $\mathrm{H}$ & 1.241962000 & 2.374178000 & -0.480286000 \\
\hline $\mathrm{H}$ & 3.810296000 & -1.055950000 & -0.032387000 \\
\hline $\mathrm{H}$ & 1.815940000 & -2.465561000 & 0.424413000 \\
\hline $\mathrm{H}$ & 3.515624000 & 1.363802000 & -0.546700000 \\
\hline $\mathrm{N}$ & -0.978162000 & 1.282979000 & 0.234247000 \\
\hline $\mathrm{O}$ & -1.507043000 & 1.147313000 & 1.324826000 \\
\hline $\mathrm{O}$ & -1.347568000 & 2.018941000 & -0.664886000 \\
\hline \multicolumn{4}{|c|}{ ts (cis/anti - trans/anti) - 2-NO $\mathrm{NO}_{6} \mathrm{H}_{4} \mathrm{NOO}$} \\
\hline $\mathrm{C}$ & 0.507517000 & 0.034424000 & -0.170956000 \\
\hline $\mathrm{C}$ & 1.216763000 & 1.272810000 & -0.244996000 \\
\hline $\mathrm{C}$ & 0.549309000 & 2.473485000 & -0.162922000 \\
\hline $\mathrm{C}$ & -0.838447000 & 2.513815000 & 0.015059000 \\
\hline $\mathrm{C}$ & -1.565568000 & 1.326120000 & 0.099905000 \\
\hline $\mathrm{C}$ & -0.915594000 & 0.119732000 & -0.015817000 \\
\hline $\mathbf{N}$ & 1.052584000 & -1.188368000 & -0.162122000 \\
\hline $\mathrm{O}$ & 2.526579000 & -1.071879000 & -0.301850000 \\
\hline
\end{tabular}




\begin{tabular}{|c|c|c|c|}
\hline $\mathrm{O}$ & 3.092169000 & -0.828908000 & 0.831582000 \\
\hline $\mathrm{H}$ & 2.292569000 & 1.250912000 & -0.354337000 \\
\hline $\mathrm{H}$ & 1.110135000 & 3.399583000 & -0.227671000 \\
\hline $\mathrm{H}$ & -2.639387000 & 1.327122000 & 0.245819000 \\
\hline $\mathrm{H}$ & -1.353816000 & 3.465253000 & 0.085839000 \\
\hline $\mathrm{N}$ & -1.747228000 & -1.098270000 & 0.001852000 \\
\hline $\mathrm{O}$ & -1.490884000 & -1.965836000 & -0.813016000 \\
\hline $\mathrm{O}$ & -2.661723000 & -1.118217000 & 0.814608000 \\
\hline \multicolumn{4}{|c|}{ ts $\left(\right.$ cis/syn - cis/anti) - 2- $\mathrm{NO}_{2} \mathrm{C}_{6} \mathrm{H}_{4} \mathrm{NOO}$} \\
\hline $\mathrm{C}$ & 0.074620000 & -0.672849000 & -0.397705000 \\
\hline $\mathrm{C}$ & 1.095212000 & -1.622006000 & -0.387428000 \\
\hline $\mathrm{C}$ & 2.387017000 & -1.252857000 & -0.038572000 \\
\hline $\mathrm{C}$ & 2.685219000 & 0.069548000 & 0.277652000 \\
\hline $\mathrm{C}$ & 1.683403000 & 1.026301000 & 0.250035000 \\
\hline $\mathrm{C}$ & 0.390061000 & 0.649391000 & -0.082694000 \\
\hline $\mathrm{N}$ & -1.194883000 & -1.100677000 & -0.892469000 \\
\hline $\mathrm{O}$ & -1.989541000 & -1.538592000 & 0.007213000 \\
\hline $\mathrm{O}$ & -1.677405000 & -1.456794000 & 1.268457000 \\
\hline $\mathrm{H}$ & 0.857765000 & -2.646365000 & -0.651140000 \\
\hline $\mathrm{H}$ & 3.167476000 & -2.006174000 & -0.021436000 \\
\hline $\mathrm{H}$ & 3.696779000 & 0.355034000 & 0.542142000 \\
\hline $\mathrm{H}$ & 1.871198000 & 2.067189000 & 0.483265000 \\
\hline $\mathrm{N}$ & -0.664502000 & 1.674540000 & -0.086337000 \\
\hline $\mathrm{O}$ & -1.813755000 & 1.286364000 & -0.251462000 \\
\hline $\mathrm{O}$ & -0.328137000 & 2.837534000 & 0.072178000 \\
\hline \multicolumn{4}{|c|}{ ts $(\operatorname{trans} / \mathrm{syn}-\operatorname{trans} / \mathrm{anti})-2-\mathrm{NO}_{2} \mathrm{C}_{6} \mathrm{H}_{4} \mathrm{NOO}$} \\
\hline $\mathrm{N}$ & 1.397108000 & -0.770881000 & 0.605204000 \\
\hline $\mathrm{O}$ & 2.081606000 & -0.817615000 & -0.501227000 \\
\hline $\mathrm{O}$ & 3.357183000 & -0.911339000 & -0.380881000 \\
\hline $\mathrm{C}$ & 0.018703000 & -0.702584000 & 0.256867000 \\
\hline
\end{tabular}



$\begin{array}{llll}\text { C } & -0.671421000 & 0.495447000 & 0.037226000\end{array}$
$\begin{array}{llll}\text { C } & -2.039117000 & 0.505922000 & -0.202479000\end{array}$
$\begin{array}{llll}\mathrm{H} & -2.522735000 & 1.460361000 & -0.370272000\end{array}$
C $\quad-2.741081000 \quad-0.688711000 \quad-0.216802000$
H $\quad-3.809407000 \quad-0.683566000 \quad-0.400051000$
$\begin{array}{llll}\text { C } & -2.071315000 & -1.887688000 & 0.008804000\end{array}$
H $\quad-2.615241000 \quad-2.826200000 \quad-0.000922000$
$\begin{array}{llll}\text { C } & -0.704272000 & -1.895089000 & 0.249561000\end{array}$
$\begin{array}{llll}\mathrm{H} & -0.173636000 & -2.822109000 & 0.436726000\end{array}$
$\begin{array}{llll}\mathrm{N} & 0.035544000 & 1.792185000 & 0.084550000\end{array}$
$\begin{array}{llll}\text { O } & 1.201567000 & 1.786662000 & 0.454980000\end{array}$
$\begin{array}{llll}\text { O } & -0.597422000 & 2.787116000 & -0.234474000\end{array}$ 


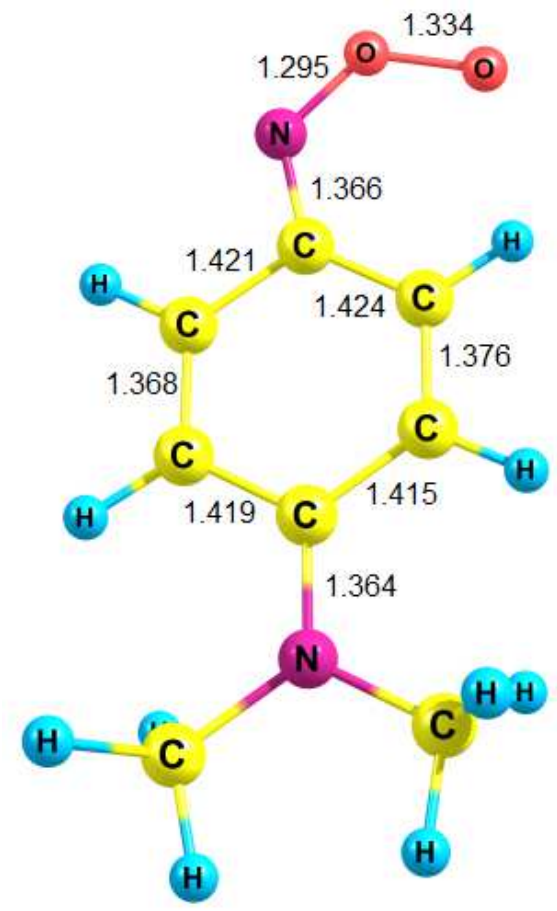

$\mathbf{a}$
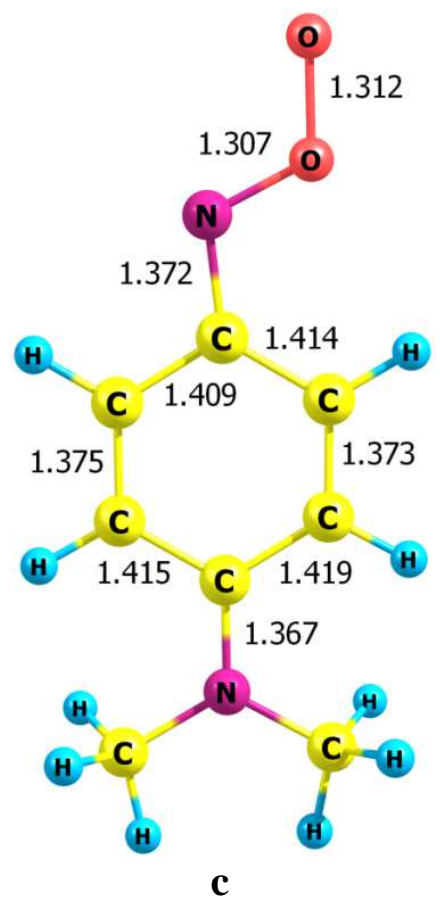

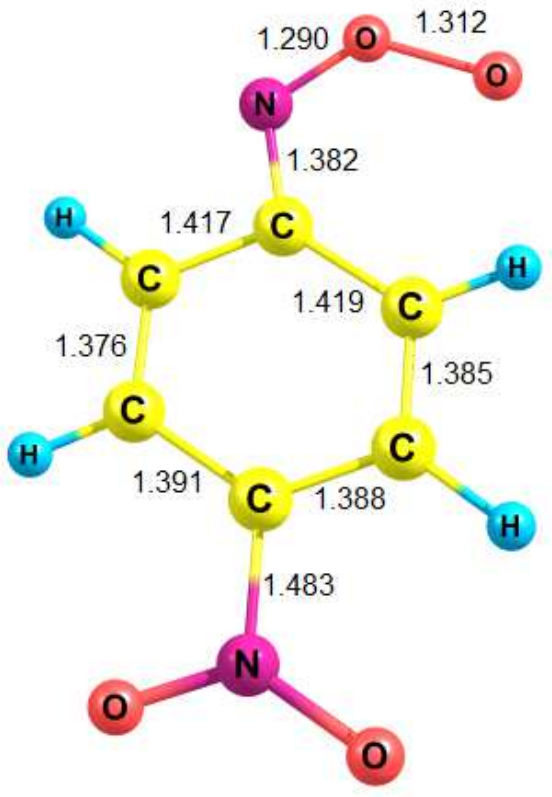

b

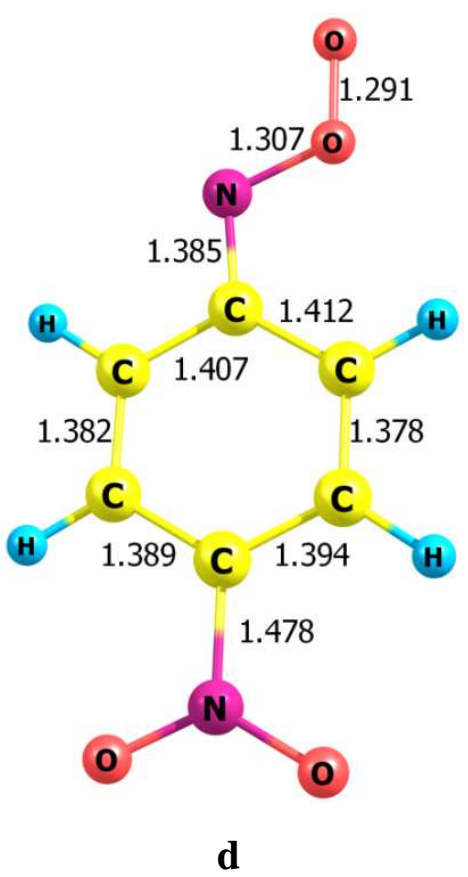

Figure S1. Geometrical parameters of cis- and trans-isomers of para- $\mathrm{Me}_{2} \mathrm{NC}_{6} \mathrm{H}_{4} \mathrm{NOO}$ and para$\mathrm{NO}_{2} \mathrm{C}_{6} \mathrm{H}_{4} \mathrm{NOO}$. Calculation in the M06-L/6-311+G(d,p) approximation 
S7. The analytic solution of differential equations describing the kinetics of the system

$$
a \rightleftharpoons b \rightarrow c
$$

The system of differential equations:

$$
\begin{aligned}
\text { sys }: & =\frac{\mathrm{d}}{\mathrm{d} x} a(x)=-k 1 \cdot a(x)+k 2 \cdot b(x), \frac{\mathrm{d}}{\mathrm{d} x} b(x)=k 1 \cdot a(x)-(k 2 \\
& +k 3) \cdot b(x), \frac{\mathrm{d}}{\mathrm{d} x} c(x)=k 3 \cdot b(x)
\end{aligned}
$$

\section{Initial conditions:}

$F:=$ dsolve $([\operatorname{sys}, a(0)=a, b(0)=b, c(0)=0])$ assuming $k 1>0, k 2$ $>0, k 3>0$ :

\section{Change of variables:}

$$
\begin{aligned}
& \text { simplify }\left(\operatorname { s u b s } \left(\left\{\sqrt{k 3^{2}+2 k 3 k 2-2 k 1 k 3+k 2^{2}+2 k 2 k 1+k 1^{2}}\right.\right.\right. \\
& \quad=w, k 1+k 2+k 3=z\}, e 1 \cdot \operatorname{rhs}(F[1])+e 2 \cdot r h s(F[2])+e 3 \\
& \quad \cdot \operatorname{rhs}(F[3])))
\end{aligned}
$$

Here $F[1]=a(x), F[2]=b(x), F[3]-=c(x)$

$$
\begin{aligned}
& \mathbf{E X P 1}=\frac{1}{2}(w-z) x \\
& \mathbf{E X P 2}=-\frac{1}{2}(w+z) x
\end{aligned}
$$

$$
\mathbf{A 0}=e 3(a+b)
$$

$\mathbf{A 1}=$

$$
\begin{aligned}
& \frac{1}{\mathrm{e}^{\frac{1}{2}(w-z) x}}\left(\text { simplify } \left(\frac{1}{4} \frac{1}{k l w}(e 1 z(b(w-z)+2 k l(b\right.\right. \\
& +a)) \mathrm{e}^{\left.\frac{1}{2}(w-z) x\right)}+\frac{1}{4} \frac{e l \mathrm{e}^{\frac{1}{2}(w-z) x} b(w-z)}{k l} \\
& +\frac{1}{2} \frac{e l \mathrm{e}^{\frac{1}{2}(w-z) x} k l(b+a)}{k l}+\frac{1}{2} e 2 \mathrm{e}^{\frac{1}{2}(w-z) x} b \\
& -\frac{1}{2} \frac{e 2 \mathrm{e}^{\frac{1}{2}(w-z) x} b z}{w}+\frac{e 2 \mathrm{e}^{\frac{1}{2}(w-z) x} k l(b+a)}{w} \\
& +\frac{e 3 k 3 \mathrm{e}^{\frac{1}{2}(w-z) x}}{w-z}-\frac{e 3 k 3 \mathrm{e}^{\frac{1}{2}(w-z) x} b z}{w(w-z)} \\
& \left.+\frac{2 e 3 k 3 \mathrm{e}^{\frac{1}{2}(w-z) x}}{w(w-z)}\right)
\end{aligned}
$$


$\mathbf{A 1}($ simplified $)=\frac{1}{4} \frac{1}{k l w}(e l z(b(w-z)+2 k l(b+a))+e l b w(w-z)$

$$
\begin{aligned}
& +2 e 1 k l w(b+a)+2 e 2 b k l(w+z)-4 e 2 b k 1 w z \\
& \left.+4 e 2 k l^{2}(b+a)+4 e 3 k 3 b k 1+\frac{8 e 3 k 3 k l(b+a) k 1}{(w-z)}\right)
\end{aligned}
$$

$$
\begin{aligned}
& \text { A2 = } \\
& \frac{1}{\mathrm{e}^{-\frac{1}{2}(w+z) x}}\left(\text { simplify } \left(-\frac{1}{2} \frac{e l \mathrm{e}^{-\frac{1}{2}(w+z) x} k 3 k 1(b+a)}{k 1 w}\right.\right. \\
& +\frac{1}{4} \frac{e l \mathrm{e}^{-\frac{1}{2}(w+z) x} k 3 b(w+z)}{k 1 w} \\
& -\frac{1}{2} \frac{e 1 \mathrm{e}^{-\frac{1}{2}(w+z) x} k 2 k 1(b+a)}{k 1 w} \\
& +\frac{1}{4} \frac{e 1 \mathrm{e}^{-\frac{1}{2}(w+z) x} k 2 b(w+z)}{k 1 w} \\
& +\frac{1}{2} \frac{e l \mathrm{e}^{-\frac{1}{2}(w+z) x} k 1(b+a)}{w} \\
& -\frac{1}{4} \frac{e l \mathrm{e}^{-\frac{1}{2}(w+z) x} b(w+z)}{w} \\
& +\frac{1}{2} \frac{e l \mathrm{e}^{-\frac{1}{2}(w+z) x} k l(b+a)}{k 1} \\
& -\frac{1}{4} \frac{e l \mathrm{e}^{-\frac{1}{2}(w+z) x} b(w+z)}{k 1} \\
& -\frac{e 2 \mathrm{e}^{-\frac{1}{2}(w+z) x} k l(b+a)}{w} \\
& +\frac{1}{2} \frac{e 2 \mathrm{e}^{-\frac{1}{2}(w+z) x} b(w+z)}{w} \\
& +\frac{2 e 3 k 3 \mathrm{e}^{-\frac{1}{2}(w+z) x} k I(b+a)}{w(w+z)} \\
& \left.-\frac{e 3 k 3 \mathrm{e}^{-\frac{1}{2}(w+z) x} b(w+z)}{w(w+z)}\right) ;
\end{aligned}
$$


A2 $($ simplified $)=$

$$
\begin{aligned}
& \frac{1}{4} \frac{1}{k l w}(-2 e l k 3 k l(b+a)+e l k 3 b(w+z)-2 e l k 2 k 1(b \\
& +a)+e 1 k 2 b(w+z)+2 e 1 k 1^{2}(b+a)-e 1 b(w+z) k 1 \\
& +2 e 1 k l w(b+a)-e 1 b w(w+z)-4 e 2 k 1(b+a) k 1 \\
& \left.+2 e 2 b(w+z) k 1+\frac{8 e 3 k 3 k 1(b+a) k 1}{(w+z)}-4 e 3 k 3 b k 1\right)
\end{aligned}
$$


Table S3. Effect of solvent on the structure $\Delta r(\AA)$, reaction enthalpies $\Delta H^{\circ}$ and activation enthalpies $\Delta H^{\neq}(\mathrm{kJ} / \mathrm{mol})$ for the conformational transitions of para-phenylnitroso oxides. Calculation by the mPWPW91/6-311+G(d,p) + IEFPCM approximation.*

\begin{tabular}{|c|c|c|c|c|c|c|c|c|}
\hline $\mathrm{R}$ & Solvent & $\Delta r_{N-O}$ & $\Delta r_{O-O}$ & $\Delta r_{C-N}$ & $\Delta\left(r_{N-O}-r_{O-O}\right)$ & $\Delta H^{\circ}$ & $\Delta H_{\text {trans } \rightarrow \text { cis }}^{\neq}$ & $\Delta H_{\text {syn } \rightarrow \text { anti }}^{\neq}$ \\
\hline \multirow{8}{*}{$-\mathrm{NMe}_{2}$} & \multirow{3}{*}{ hexane } & 0.001 & 0.009 & -0.004 & -0.008 & \multirow{3}{*}{-8.9} & \multirow{3}{*}{$68.7(62 \pm 0.3)$} & 64.8 \\
\hline & & & & & & & & \\
\hline & & 0.000 & 0.011 & -0.004 & -0.011 & & & 61.4 \\
\hline & \multirow{3}{*}{ benzene } & 0.001 & 0.012 & -0.005 & -0.011 & \multirow{3}{*}{-8.2} & \multirow{3}{*}{72.9} & 66.5 \\
\hline & & & & & & & & \\
\hline & & -0.001 & 0.014 & -0.005 & -0.015 & & & 63.5 \\
\hline & \multirow{2}{*}{ acetonitrile } & 0.003 & 0.031 & -0.013 & -0.028 & \multirow{2}{*}{-3.0} & \multirow{2}{*}{$77.1(13.7 \pm 0.3)$} & 77.8 \\
\hline & & 0.001 & 0.037 & -0.015 & -0.036 & & & 79.3 \\
\hline \multirow{6}{*}{$-\mathrm{OMe}$} & \multirow[b]{2}{*}{ hexane } & 0.000 & 0.007 & -0.003 & -0.008 & \multirow[b]{2}{*}{-8.3} & \multirow[b]{2}{*}{$69.6(66.9 \pm 0.7)$} & 50.1 \\
\hline & & -0.002 & 0.009 & -0.003 & -0.010 & & & 47.7 \\
\hline & \multirow[b]{2}{*}{ benzene } & -0.002 & 0.010 & -0.003 & -0.011 & \multirow[b]{2}{*}{-7.6} & \multirow[b]{2}{*}{$70.4(67.2 \pm 0.9)$} & 50.8 \\
\hline & & -0.002 & 0.011 & -0.004 & -0.013 & & & 48.8 \\
\hline & \multirow[b]{2}{*}{ acetonitrile } & -0.001 & 0.022 & -0.009 & -0.023 & \multirow[b]{2}{*}{-2.9} & \multirow[b]{2}{*}{$76.9(70.4 \pm 0.6)$} & 55.3 \\
\hline & & -0.003 & 0.027 & -0.011 & -0.029 & & & 56.9 \\
\hline
\end{tabular}




\begin{tabular}{|c|c|c|c|c|c|c|c|c|}
\hline \multirow{6}{*}{$-\mathrm{Me}$} & \multirow[b]{2}{*}{ hexane } & -0.001 & 0.006 & -0.002 & -0.007 & \multirow[b]{2}{*}{-4.2} & \multirow[b]{2}{*}{71.2} & 38.8 \\
\hline & & -0.002 & 0.007 & -0.002 & -0.009 & & & 40.5 \\
\hline & \multirow{2}{*}{ benzene } & -0.001 & 0.008 & -0.003 & -0.009 & \multirow[b]{2}{*}{-3.7} & \multirow[b]{2}{*}{71.8} & 39.3 \\
\hline & & -0.003 & 0.009 & -0.003 & -0.012 & & & 41.2 \\
\hline & \multirow{2}{*}{ acetonitrile } & -0.002 & 0.018 & -0.007 & -0.020 & \multirow{2}{*}{0.0} & \multirow{2}{*}{76.7} & 42.0 \\
\hline & & -0.006 & 0.022 & -0.008 & -0.028 & & & 46.6 \\
\hline \multirow{6}{*}{$-\mathrm{Br}$} & \multirow[b]{2}{*}{ hexane } & 0.000 & 0.004 & -0.002 & -0.004 & \multirow[b]{2}{*}{-4.2} & \multirow[b]{2}{*}{70.5} & 37.1 \\
\hline & & -0.002 & 0.006 & -0.002 & -0.008 & & & 39.3 \\
\hline & \multirow[b]{2}{*}{ benzene } & -0.001 & 0.006 & -0.002 & -0.007 & \multirow[b]{2}{*}{-3.6} & \multirow[b]{2}{*}{71.1} & 37.3 \\
\hline & & -0.003 & 0.008 & -0.002 & -0.011 & & & 39.8 \\
\hline & \multirow[b]{2}{*}{ acetonitrile } & -0.002 & 0.015 & -0.006 & -0.017 & \multirow[b]{2}{*}{0.2} & \multirow[b]{2}{*}{75.6} & 38.4 \\
\hline & & -0.006 & 0.020 & -0.006 & -0.026 & & & 43.4 \\
\hline \multirow{3}{*}{$-\mathrm{H}$} & \multirow[b]{2}{*}{ hexane } & -0.002 & 0.006 & -0.002 & -0.007 & \multirow[b]{2}{*}{-2.9} & \multirow[b]{2}{*}{72.3} & 33.2 \\
\hline & & -0.003 & 0.006 & -0.002 & -0.009 & & & 36.3 \\
\hline & benzene & -0.002 & 0.007 & -0.002 & -0.009 & -2.4 & 72.9 & 33.4 \\
\hline
\end{tabular}




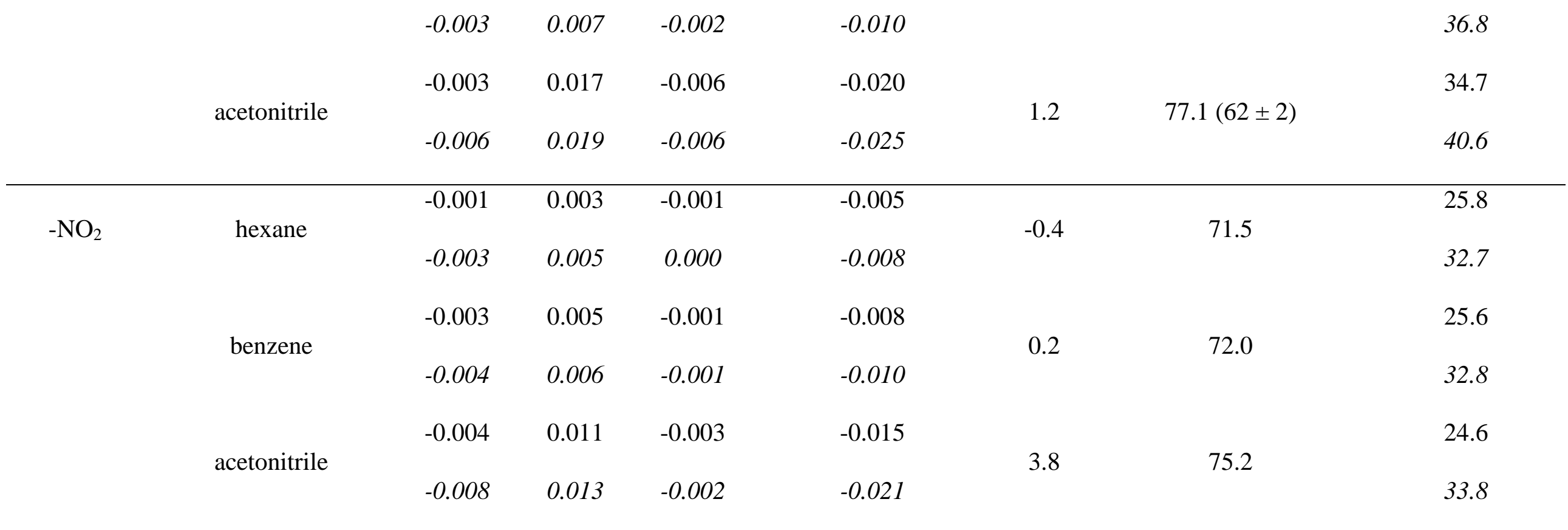

* The numbers in parentheses indicate the experimental activation energies of trans-isomer decay. The results for the trans-isomers are given in Italic; $\Delta r=$ $r$ (solvent) $-r$ (gas); $\Delta H^{\circ}=H^{\circ}$ cis $-H^{\circ}$ trans. 
Table S4. Effect of solvent on the structure $\Delta r(\AA)$, reaction enthalpies $\Delta H^{\circ}$ and activation enthalpies $\Delta H^{\neq}(\mathrm{kJ} / \mathrm{mol})$ for the conformational transitions of para-phenylnitroso oxides. Calculation by the OLYP $/ 6-311+\mathrm{G}(\mathrm{d}, \mathrm{p})+\mathrm{IEFPCM}$ approximation.*

\begin{tabular}{|c|c|c|c|c|c|c|c|c|}
\hline $\mathrm{R}$ & Solvent & $\Delta r_{N-O}$ & $\Delta r_{O-O}$ & $\Delta r_{C-N}$ & $\Delta\left(r_{N-O}-r_{O-O}\right)$ & $\Delta H^{\circ}$ & $\Delta H_{\text {trans } \rightarrow \text { cis }}^{\neq}$ & $\Delta H_{\text {syn } \rightarrow \text { anti }}^{\neq}$ \\
\hline \multirow{8}{*}{$-\mathrm{NMe}_{2}$} & \multirow{3}{*}{ hexane } & 0.001 & 0.009 & -0.004 & -0.009 & \multirow{3}{*}{-2.3} & \multirow{3}{*}{$71.4(62 \pm 0.3)$} & 58.7 \\
\hline & & & & & & & & \\
\hline & & -0.001 & 0.011 & -0.004 & -0.011 & & & 58.4 \\
\hline & \multirow{3}{*}{ benzene } & 0.001 & 0.012 & -0.005 & -0.011 & \multirow{3}{*}{-1.6} & \multirow{3}{*}{72.4} & 60.3 \\
\hline & & & & & & & & \\
\hline & & -0.001 & 0.014 & -0.005 & -0.014 & & & 60.5 \\
\hline & \multirow{2}{*}{ acetonitrile } & 0.003 & 0.030 & -0.013 & -0.027 & \multirow{2}{*}{3.5} & \multirow{2}{*}{$80.7(13.7 \pm 0.3)$} & 71.5 \\
\hline & & 0.001 & 0.036 & -0.015 & -0.035 & & & 75.8 \\
\hline \multirow{6}{*}{$-\mathrm{OMe}$} & \multirow[b]{2}{*}{ hexane } & 0.000 & 0.007 & -0.003 & -0.007 & \multirow[b]{2}{*}{-1.8} & \multirow[b]{2}{*}{$72.7(66.9 \pm 0.7)$} & 45.0 \\
\hline & & -0.002 & 0.008 & -0.003 & -0.010 & & & 45.5 \\
\hline & \multirow[b]{2}{*}{ benzene } & -0.001 & 0.009 & -0.004 & -0.009 & \multirow[b]{2}{*}{-1.2} & \multirow[b]{2}{*}{$73.5(67.2 \pm 0.9)$} & 45.7 \\
\hline & & -0.002 & 0.010 & -0.004 & -0.013 & & & 46.6 \\
\hline & \multirow{2}{*}{ acetonitrile } & -0.001 & 0.021 & -0.009 & -0.022 & \multirow{2}{*}{3.5} & \multirow{2}{*}{$79.8(70.4 \pm 0.6)$} & 50.2 \\
\hline & & -0.004 & 0.026 & -0.010 & -0.030 & & & 54.6 \\
\hline
\end{tabular}




\begin{tabular}{|c|c|c|c|c|c|c|c|c|}
\hline \multirow{6}{*}{-Me } & \multirow[b]{2}{*}{ hexane } & -0.001 & 0.006 & -0.002 & -0.007 & \multirow[b]{2}{*}{1.8} & \multirow[b]{2}{*}{74.2} & 33.9 \\
\hline & & -0.002 & 0.007 & -0.002 & -0.009 & & & 38.1 \\
\hline & \multirow[b]{2}{*}{ benzene } & -0.001 & 0.007 & -0.003 & -0.008 & \multirow[b]{2}{*}{-3.7} & \multirow[b]{2}{*}{74.8} & 34.3 \\
\hline & & -0.003 & 0.009 & -0.003 & -0.012 & & & 38.8 \\
\hline & \multirow{2}{*}{ acetonitrile } & -0.002 & 0.018 & -0.007 & -0.020 & \multirow{2}{*}{6.1} & \multirow{2}{*}{79.7} & 37.0 \\
\hline & & -0.005 & 0.021 & -0.008 & -0.027 & & & 44.0 \\
\hline \multirow{6}{*}{$-\mathrm{Br}$} & \multirow[b]{2}{*}{ hexane } & -0.001 & 0.005 & -0.002 & -0.006 & \multirow[b]{2}{*}{1.8} & \multirow[b]{2}{*}{73.5} & 32.2 \\
\hline & & -0.003 & 0.006 & -0.001 & -0.009 & & & 36.9 \\
\hline & \multirow[b]{2}{*}{ benzene } & 0.007 & -0.015 & 0.004 & 0.022 & \multirow[b]{2}{*}{2.4} & \multirow[b]{2}{*}{74.1} & 32.3 \\
\hline & & -0.003 & 0.008 & -0.002 & -0.011 & & & 37.3 \\
\hline & \multirow{2}{*}{ acetonitrile } & -0.003 & 0.015 & -0.005 & -0.018 & \multirow{2}{*}{6.2} & \multirow{2}{*}{78.6} & 33.3 \\
\hline & & -0.007 & 0.019 & -0.005 & -0.026 & & & 40.8 \\
\hline \multirow{3}{*}{$-\mathrm{H}$} & \multirow[b]{2}{*}{ hexane } & -0.001 & 0.005 & -0.001 & -0.006 & \multirow[b]{2}{*}{3.1} & \multirow[b]{2}{*}{75.4} & 28.6 \\
\hline & & -0.003 & 0.006 & -0.001 & -0.010 & & & 34.1 \\
\hline & benzene & -0.001 & 0.006 & -0.001 & -0.007 & 3.6 & 76.0 & 28.8 \\
\hline
\end{tabular}




\begin{tabular}{|c|c|c|c|c|c|c|c|c|}
\hline & & -0.004 & 0.008 & -0.002 & -0.012 & & & 34.6 \\
\hline & \multirow[b]{2}{*}{ acetonitrile } & -0.003 & 0.015 & -0.005 & -0.018 & \multirow[b]{2}{*}{7.1} & \multirow[b]{2}{*}{$80.2(62 \pm 2)$} & 30.1 \\
\hline & & -0.007 & 0.019 & -0.006 & & & & 38.3 \\
\hline \multirow{8}{*}{$-\mathrm{NO}_{2}$} & \multirow{3}{*}{ hexane } & -0.002 & 0.003 & -0.001 & -0.005 & \multirow{3}{*}{5.2} & \multirow{3}{*}{74.5} & 21.6 \\
\hline & & & & & & & & \\
\hline & & -0.003 & 0.005 & 0.000 & -0.008 & & & 30.5 \\
\hline & \multirow{3}{*}{ benzene } & -0.002 & 0.004 & -0.001 & -0.006 & \multirow{3}{*}{5.8} & \multirow{3}{*}{75.0} & 21.5 \\
\hline & & & & & & & & \\
\hline & & -0.004 & 0.006 & 0.000 & -0.010 & & & 30.6 \\
\hline & \multirow[b]{2}{*}{ acetonitrile } & -0.004 & 0.011 & -0.003 & -0.015 & \multirow[b]{2}{*}{9.3} & \multirow[b]{2}{*}{78.1} & 20.6 \\
\hline & & -0.008 & 0.014 & -0.002 & -0.022 & & & 31.6 \\
\hline
\end{tabular}

* The numbers in parentheses indicate the experimental activation energies of trans-isomer decay. The results for the trans-isomers are given in Italic; $\Delta r=$ $r$ (solvent) $-r$ (gas); $\Delta H^{\circ}=H^{\circ}$ cis $-H^{\circ}$ trans. 
Table S5. Effect of solvent on the structure $\Delta r(\AA)$, reaction enthalpies $\Delta H^{\circ}$ and activation enthalpies $\Delta H^{\ddagger}(\mathrm{kJ} / \mathrm{mol})(\mathrm{kJ} / \mathrm{mol})$ for the conformational transitions of para-phenylnitroso oxides. Calculation by the HCTH/6-311+G(d,p) + IEFPCM approximation.*

\begin{tabular}{|c|c|c|c|c|c|c|c|c|}
\hline $\mathrm{R}$ & Solvent & $\Delta r_{N-O}$ & $\Delta r_{O-O}$ & $\Delta r_{C-N}$ & $\Delta\left(r_{N-O}-r_{O-O}\right)$ & $\Delta H^{\circ}$ & $\Delta H_{\text {trans } \rightarrow \text { cis }}^{\neq}$ & $\Delta H_{\text {syn } \rightarrow \text { anti }}^{f}$ \\
\hline \multirow{8}{*}{$-\mathrm{NMe}_{2}$} & \multirow{3}{*}{ hexane } & 0.000 & 0.009 & -0.004 & -0.009 & \multirow{3}{*}{-3.4} & \multirow{3}{*}{$72.7(62 \pm 0.3)$} & 59.4 \\
\hline & & & & & & & & \\
\hline & & -0.001 & 0.010 & -0.004 & -0.011 & & & 58.8 \\
\hline & \multirow{3}{*}{ benzene } & 0.001 & 0.012 & -0.005 & -0.011 & \multirow{3}{*}{-2.7} & \multirow{3}{*}{73.6} & 61.0 \\
\hline & & & & & & & & \\
\hline & & -0.001 & 0.013 & -0.005 & -0.014 & & & 60.8 \\
\hline & \multirow{2}{*}{ acetonitrile } & 0.002 & 0.030 & -0.012 & -0.028 & \multirow{2}{*}{3.5} & \multirow{2}{*}{$81.5(13.7 \pm 0.3)$} & 71.9 \\
\hline & & 0.000 & 0.035 & -0.014 & -0.035 & & & 75.8 \\
\hline \multirow{8}{*}{$-\mathrm{OMe}$} & \multirow{3}{*}{ hexane } & 0.000 & 0.007 & -0.003 & -0.007 & \multirow{3}{*}{-2.7} & \multirow{3}{*}{$74.1(66.9 \pm 0.7)$} & 45.7 \\
\hline & & & & & & & & \\
\hline & & -0.002 & 0.008 & -0.003 & -0.010 & & & 46.1 \\
\hline & \multirow{3}{*}{ benzene } & -0.001 & 0.009 & -0.003 & -0.010 & \multirow{3}{*}{-2.0} & \multirow{3}{*}{$74.9(67.2 \pm 0.9)$} & 46.4 \\
\hline & & & & & & & & \\
\hline & & -0.002 & 0.010 & -0.004 & -0.012 & & & 47.1 \\
\hline & \multirow[b]{2}{*}{ acetonitrile } & -0.001 & 0.021 & -0.009 & -0.022 & \multirow[b]{2}{*}{2.6} & \multirow[b]{2}{*}{$81.1(70.4 \pm 0.6)$} & 50.7 \\
\hline & & -0.004 & 0.025 & -0.010 & -0.029 & & & 55.0 \\
\hline
\end{tabular}




\begin{tabular}{|c|c|c|c|c|c|c|c|c|}
\hline \multirow{6}{*}{-Me } & \multirow[b]{2}{*}{ hexane } & -0.001 & 0.006 & -0.002 & -0.006 & \multirow[b]{2}{*}{1.1} & \multirow[b]{2}{*}{75.4} & 34.7 \\
\hline & & -0.002 & 0.007 & -0.002 & -0.009 & & & 38.9 \\
\hline & \multirow[b]{2}{*}{ benzene } & -0.001 & 0.007 & -0.003 & -0.008 & \multirow[b]{2}{*}{1.6} & \multirow[b]{2}{*}{76.0} & 35.1 \\
\hline & & -0.003 & 0.009 & -0.003 & -0.011 & & & 39.6 \\
\hline & \multirow{2}{*}{ acetonitrile } & -0.002 & 0.018 & -0.007 & -0.020 & \multirow{2}{*}{5.3} & \multirow{2}{*}{80.7} & 37.7 \\
\hline & & -0.006 & 0.021 & -0.007 & -0.027 & & & 44.7 \\
\hline \multirow{6}{*}{$-\mathrm{Br}$} & \multirow[b]{2}{*}{ hexane } & -0.001 & 0.005 & -0.002 & -0.006 & \multirow[b]{2}{*}{1.0} & \multirow[b]{2}{*}{74.7} & 33.0 \\
\hline & & -0.003 & 0.006 & -0.001 & -0.008 & & & 37.6 \\
\hline & \multirow[b]{2}{*}{ benzene } & 0.006 & -0.016 & 0.004 & 0.023 & \multirow[b]{2}{*}{1.6} & \multirow[b]{2}{*}{75.3} & 33.1 \\
\hline & & -0.003 & 0.008 & -0.002 & -0.011 & & & 38.1 \\
\hline & \multirow{2}{*}{ acetonitrile } & -0.003 & 0.015 & -0.005 & -0.018 & \multirow{2}{*}{5.4} & \multirow{2}{*}{79.7} & 34.1 \\
\hline & & -0.007 & 0.018 & -0.005 & -0.025 & & & 41.5 \\
\hline \multirow{3}{*}{$-\mathrm{H}$} & \multirow[b]{2}{*}{ hexane } & -0.001 & 0.007 & -0.001 & -0.008 & \multirow[b]{2}{*}{2.4} & \multirow[b]{2}{*}{64.8} & 29.2 \\
\hline & & -0.002 & 0.006 & -0.002 & -0.008 & & & 34.8 \\
\hline & benzene & -0.001 & 0.007 & -0.001 & -0.008 & 2.9 & 77.1 & 29.4 \\
\hline
\end{tabular}




\begin{tabular}{|c|c|c|c|c|c|c|c|c|}
\hline & & -0.003 & 0.008 & -0.002 & -0.010 & & & 35.3 \\
\hline & \multirow{2}{*}{ acetonitrile } & -0.003 & 0.016 & -0.005 & -0.019 & \multirow{2}{*}{6.4} & \multirow{2}{*}{$81.3(62 \pm 2)$} & 30.7 \\
\hline & & -0.006 & 0.018 & -0.006 & -0.025 & & & 38.9 \\
\hline \multirow{6}{*}{$-\mathrm{NO}_{2}$} & \multirow[b]{2}{*}{ hexane } & -0.002 & 0.013 & -0.003 & -0.0015 & \multirow[b]{2}{*}{4.7} & \multirow[b]{2}{*}{75.8} & 21.9 \\
\hline & & -0.003 & 0.004 & 0.000 & -0.007 & & & 31.1 \\
\hline & \multirow[b]{2}{*}{ benzene } & -0.002 & 0.004 & -0.001 & -0.006 & \multirow[b]{2}{*}{5.2} & \multirow[b]{2}{*}{76.3} & 21.8 \\
\hline & & -0.004 & 0.005 & 0.000 & -0.009 & & & 31.2 \\
\hline & \multirow{2}{*}{ acetonitrile } & -0.004 & 0.010 & -0.003 & -0.014 & \multirow{2}{*}{8.9} & \multirow{2}{*}{79.7} & 20.8 \\
\hline & & -0.008 & 0.013 & -0.002 & -0.021 & & & 32.3 \\
\hline
\end{tabular}

* The numbers in parentheses indicate the experimental activation energies of trans-isomer decay. The results for the trans-isomers are given in Italic; $\Delta r=$ $r$ (solvent) $-r\left(\right.$ gas); $\Delta H^{\circ}=H^{\circ}{ }_{\text {cis }}-H^{\circ}{ }_{\text {trans }}$. 
Table S6. Effect of solvent on the reaction enthalpies $\Delta H^{\circ}$ and activation enthalpies $\Delta H^{\ddagger}(\mathrm{kJ} / \mathrm{mol})$ for the conformational transitions of orto-phenylnitroso oxides. Calculation by the mPWPW91/6-311+G(d,p) + IEFPCM approximation.

\begin{tabular}{|c|c|c|c|c|c|c|c|c|c|}
\hline \multirow[t]{2}{*}{$\mathrm{R}$} & \multirow[t]{2}{*}{ Solvent } & \multicolumn{2}{|c|}{ syn } & \multicolumn{2}{|c|}{ anti } & \multicolumn{4}{|c|}{ syn $\rightarrow$ anti } \\
\hline & & $\Delta H^{\circ}$ & $\Delta H_{\text {trans } \rightarrow \text { cis }}^{\neq}$ & $\Delta H^{\circ}$ & $\Delta H_{\text {trans } \rightarrow \text { cis }}^{\neq}$ & $\Delta H_{c i s}^{\circ} *$ & $\Delta H_{\text {trans }}^{o} * *$ & $\Delta H_{c i s}^{\neq}$ & $\Delta H_{\text {trans }}^{\ddagger}$ \\
\hline \multirow{4}{*}{$-\mathrm{NMe}_{2}$} & - & -11.7 & 69.7 & -4.2 & 62.4 & -3.4 & -11.0 & 39.5 & 30.8 \\
\hline & hexane & -8.7 & 73.2 & -2.4 & 65.9 & -4.3 & -10.6 & 43.1 & 36.3 \\
\hline & benzene & -7.9 & 74.3 & -1.9 & 66.9 & -4.5 & -10.5 & 44.0 & 37.9 \\
\hline & acetonitrile & -1.9 & 81.8 & 1.7 & 74.5 & -6.5 & -10.1 & 50.0 & 49.0 \\
\hline \multirow{4}{*}{$-\mathrm{OMe}$} & - & 4.1 & 74.6 & -5.5 & 64.3 & -9.2 & 0.4 & 22.2 & 34.3 \\
\hline & hexane & 7.3 & 77.0 & -3.4 & 66.9 & -10.4 & 0.3 & 23.9 & 38.1 \\
\hline & benzene & 8.1 & 77.7 & -2.9 & 67.6 & -10.7 & 0.2 & 24.3 & 39.2 \\
\hline & acetonitrile & 14.2 & 83.5 & 1.3 & 73.1 & -13.2 & -0.3 & 27.2 & 47.0 \\
\hline \multirow{3}{*}{$-\mathrm{Me}$} & - & 5.0 & 76.5 & -3.7 & 67.0 & -14.4 & -5.7 & 15.9 & 29.1 \\
\hline & hexane & 8.1 & 79.3 & -1.8 & 69.2 & -14.9 & -5.0 & 16.5 & 31.8 \\
\hline & benzene & 8.9 & 80.0 & -1.3 & 69.8 & -15.1 & -4.9 & 16.7 & 32.5 \\
\hline
\end{tabular}




\begin{tabular}{|c|c|c|c|c|c|c|c|c|c|}
\hline & acetonitrile & 14.3 & 85.2 & 2.1 & 74.3 & -16.0 & -3.8 & 17.8 & 37.7 \\
\hline & - & 3.3 & 77.1 & -3.6 & 66.3 & -16.0 & -9.1 & 9.4 & 21.3 \\
\hline \multirow[t]{4}{*}{$-\mathrm{Br}$} & hexane & 5.6 & 78.9 & -1.7 & 68.2 & -16.2 & -9.0 & 10.2 & 23.5 \\
\hline & benzene & 6.2 & 67.8 & -1.2 & 68.7 & -16.3 & -9.0 & 10.4 & 24.1 \\
\hline & acetonitrile & 10.6 & 94.7 & 2.8 & 72.8 & -16.9 & -9.1 & 11.7 & 28.6 \\
\hline & - & -3.7 & 75.7 & -4.7 & 68.7 & -3.3 & -2.4 & 5.8 & 16.4 \\
\hline \multirow[t]{3}{*}{$-\mathrm{NO}_{2}$} & hexane & -2.5 & 76.7 & -2.7 & 70.2 & -3.1 & -2.9 & 6.5 & 16.8 \\
\hline & benzene & -2.2 & 77.0 & -2.0 & 70.6 & -3.0 & -3.1 & 6.7 & 17.0 \\
\hline & acetonitrile & -0.2 & 79.6 & 2.4 & 73.8 & -1.9 & -4.4 & 8.8 & 18.1 \\
\hline
\end{tabular}

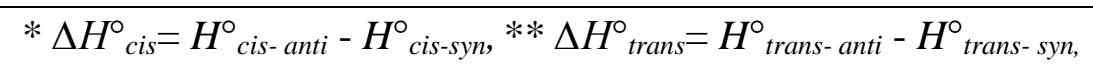


Table S7. Effect of solvent on the Gibbs free energy $\Delta \mathrm{G}^{\circ}$ of reaction and Gibbs free energy activation $\Delta \mathrm{G}^{\neq}(\mathrm{kJ} / \mathrm{mol})$ for the conformational transitions of para-phenylnitroso oxides. Calculation by the M06-L/6-311+G(d,p) + IEFPCM approximation.*

\begin{tabular}{|c|c|c|c|c|}
\hline $\mathrm{R}$ & Solvent & $\Delta G^{\circ}$ & $\Delta G_{\text {trans } \rightarrow \text { cis }}^{\neq}$ & $\Delta G_{s y n \rightarrow a n t i}^{\neq}$ \\
\hline \multirow[t]{2}{*}{$-\mathrm{NMe}_{2}$} & hexane & -9.2 & 64.3 & $\begin{array}{l}71.7 \\
71.3\end{array}$ \\
\hline & $\begin{array}{c}\text { benzene } \\
\text { acetonitrile }\end{array}$ & $\begin{array}{l}-8.5 \\
-3.3\end{array}$ & $\begin{array}{r}65.5 \\
75.2\end{array}$ & $\begin{array}{l}73.0 \\
73.1 \\
83.2 \\
87.7\end{array}$ \\
\hline \multirow[t]{3}{*}{$-\mathrm{OMe}$} & hexane & -7.7 & 63.4 & $\begin{array}{l}55.6 \\
56.9\end{array}$ \\
\hline & benzene & -7.0 & 64.3 & $\begin{array}{l}56.4 \\
58.1\end{array}$ \\
\hline & acetonitrile & -2.6 & 71.6 & $\begin{array}{l}61.7 \\
66.4\end{array}$ \\
\hline \multirow[t]{3}{*}{$-\mathrm{Me}$} & hexane & -5.0 & 60.2 & $\begin{array}{l}45.0 \\
48.6\end{array}$ \\
\hline & benzene & -4.4 & 61.0 & $\begin{array}{l}45.5 \\
49.4\end{array}$ \\
\hline & acetonitrile & -0.5 & 67.2 & $\begin{array}{l}48.5 \\
54.7\end{array}$ \\
\hline \multirow[t]{2}{*}{$-\mathrm{Br}$} & hexane & -4.3 & 62.0 & $\begin{array}{l}43.1 \\
48.6\end{array}$ \\
\hline & benzene & -3.7 & 62.8 & 43.3 \\
\hline
\end{tabular}




$\begin{array}{llll}\text { acetonitrile } & 0.1 & 67.8 & 44.6\end{array}$

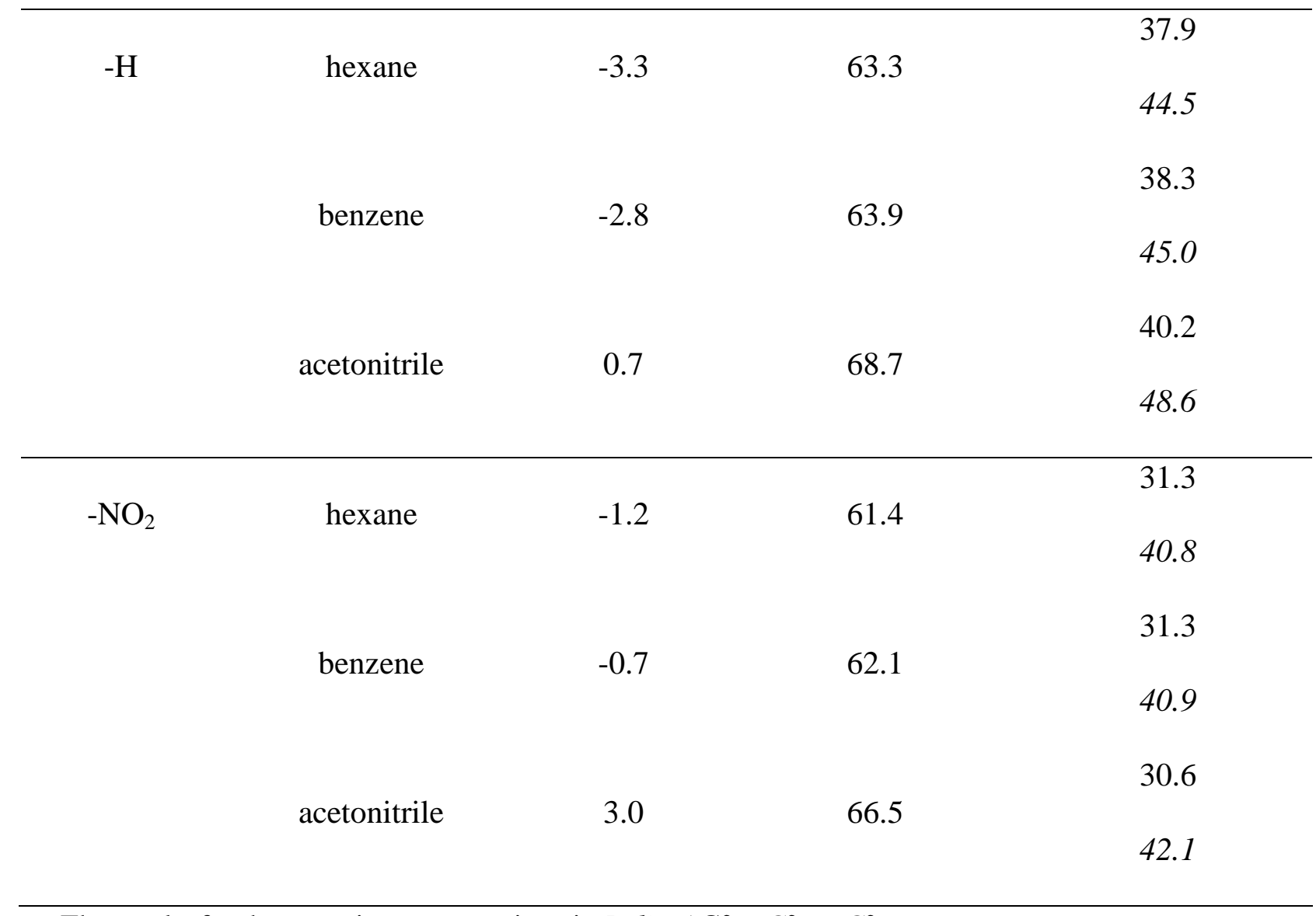

The results for the trans-isomers are given in Italic; $\Delta \mathrm{G}^{\circ}=G_{\text {cis }}^{\circ}-G_{\text {trans }}^{\circ}$. 
Table S8. Effect of solvent on the Gibbs free energy $\Delta \mathrm{G}^{\circ}$ of reaction and Gibbs free energy activation $\Delta \mathrm{G}^{\neq}(\mathrm{kJ} / \mathrm{mol})$ for the conformational transitions of orto-phenylnitroso oxides. Calculation by the M06-L/6-311+G(d,p) + IEFPCM approximation.

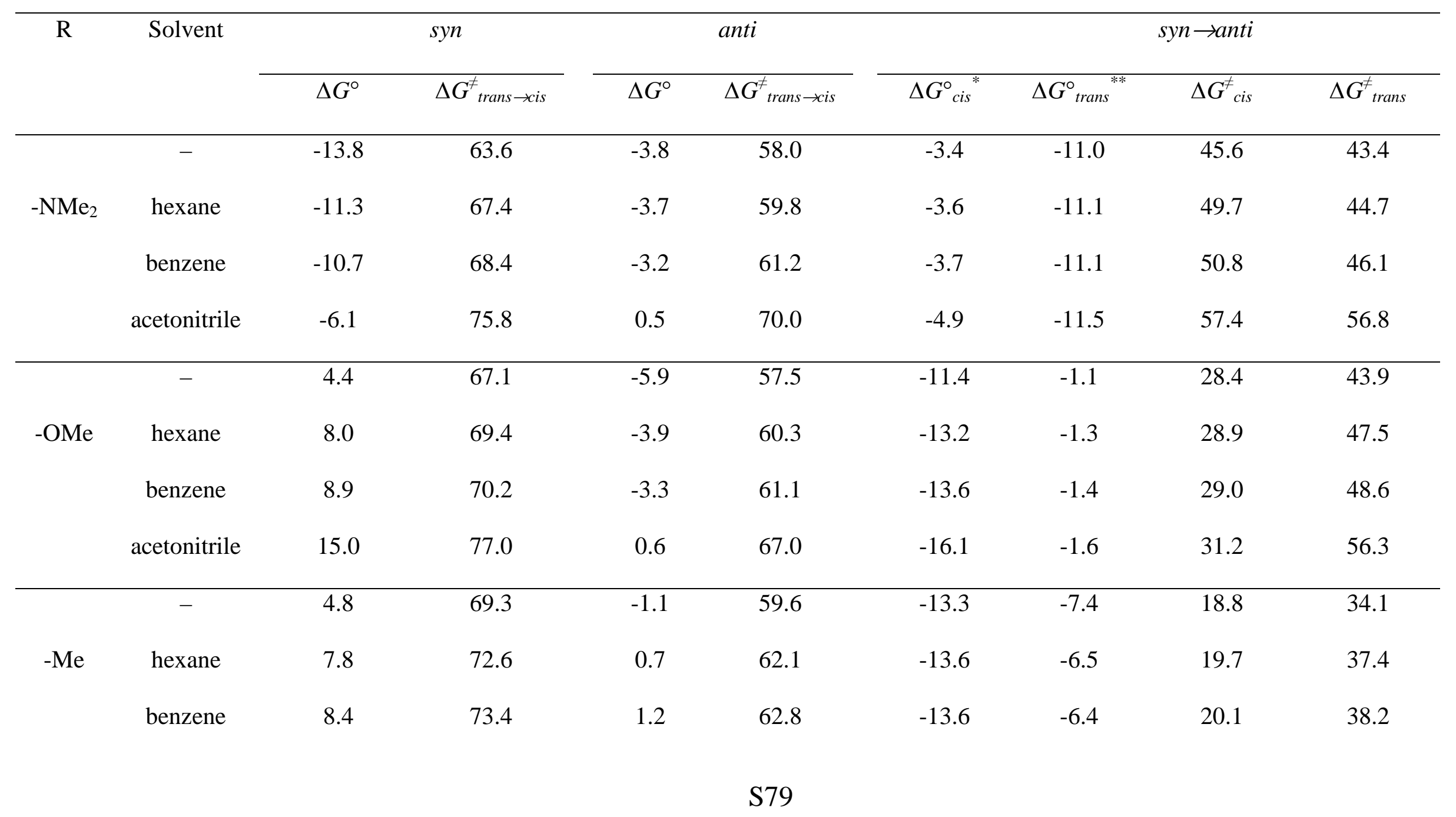




\begin{tabular}{|c|c|c|c|c|c|c|c|c|c|}
\hline & acetonitrile & 11.4 & 79.1 & 4.1 & 67.7 & -12.4 & -5.1 & 23.6 & 43.8 \\
\hline & - & 2.4 & 67.7 & -3.3 & 59.8 & -17.2 & -11.5 & 14.8 & 28.0 \\
\hline \multirow[t]{4}{*}{$-\mathrm{Br}$} & hexane & 9.1 & 74.1 & -1.7 & 61.9 & -17.8 & -6.9 & 15.4 & 35.7 \\
\hline & benzene & 2.2 & 67.1 & -1.0 & 62.7 & -17.9 & -11.4 & 15.6 & 31.9 \\
\hline & acetonitrile & 12.7 & 77.6 & 2.3 & 67.0 & -19.2 & -8.8 & 16.8 & 38.7 \\
\hline & - & -9.4 & 65.5 & -4.3 & 58.1 & 1.5 & -3.6 & 12.1 & 19.3 \\
\hline \multirow[t]{3}{*}{$-\mathrm{NO}_{2}$} & hexane & -7.7 & 67.1 & -2.5 & 60.3 & 1.4 & -3.9 & 12.7 & 21.3 \\
\hline & benzene & -7.2 & 67.7 & -1.9 & 60.9 & 1.4 & -4.0 & 12.9 & 21.5 \\
\hline & acetonitrile & -4.4 & 71.6 & 1.7 & 65.1 & 1.1 & -4.9 & 14.8 & 23.3 \\
\hline
\end{tabular}

$* \Delta G_{c i s}^{\circ}=G_{c i s-\text { anti }}^{\circ}-G_{c i s-s y n}^{\circ}, * * G_{\text {trans }}^{\circ}=G_{\text {trans- anti }}^{\circ}-G^{\circ}{ }_{\text {trans- syn }}$, 
Table S9. Comparison of relaxation times $\tau *$ of conformational transitions and life times $t$ of trans- and cis-isomers of para- $\mathrm{R}-\mathrm{C}_{6} \mathrm{H}_{4} \mathrm{NOO}$ (in benzene as the solvent)

\begin{tabular}{|c|c|c|c|c|c|c|}
\hline \multirow[t]{2}{*}{$\tau, \mathrm{c}$} & \multicolumn{6}{|c|}{$\mathrm{R}$} \\
\hline & $-\mathrm{NMe}_{2}$ & $-\mathrm{OMe}$ & $-\mathrm{Me}$ & $-\mathrm{Br}$ & $-\mathrm{H}$ & $-\mathrm{NO}_{2}$ \\
\hline$\tau_{\text {trans-cis }}$ & 0.047 & 0.028 & $6.8 \cdot 10-3$ & 0.013 & 0.02 & $6.9 \cdot 10-3$ \\
\hline$\tau_{\text {cis-cis }}$ & 0.51 & $6.2 \cdot 10-4$ & $7.6 \cdot 10-6$ & $3.1 \cdot 10-6$ & $4.1 \cdot 10-7$ & $2.4 \cdot 10-8$ \\
\hline$\tau_{\text {trans-trans }}$ & 0.53 & $1.2 \cdot 10-3$ & $3.7 \cdot 10-5$ & $3.3 \cdot 10-5$ & $6.2 \cdot 10-6$ & $1.2 \cdot 10-6$ \\
\hline $\mathrm{t}, \mathrm{c}$ & & & & & & \\
\hline$t_{\text {trans }}$ & 0.1 & 1.8 & 4.3 & 5.3 & 8.3 & - \\
\hline $\mathrm{t}_{\text {cis }}$ & 0.4 & 0.1 & 0.6 & 0.7 & 2.2 & - \\
\hline
\end{tabular}

Table S10. Comparison of relaxation times $\tau *$ of conformational transitions and life times $t$ of trans- and cis-isomers of para- $\mathrm{R}-\mathrm{C}_{6} \mathrm{H}_{4} \mathrm{NOO}$ (in acetonitrile as the solvent)

\begin{tabular}{|c|c|c|c|c|c|c|}
\hline \multirow[t]{2}{*}{$\tau, \mathrm{c}$} & \multicolumn{5}{|c|}{$\mathrm{R}$} & \multirow[b]{2}{*}{$-\mathrm{NO}_{2}$} \\
\hline & $-\mathrm{NMe}_{2}$ & $-\mathrm{OMe}$ & $-\mathrm{Me}$ & $-\mathrm{Br}$ & $-\mathrm{H}$ & \\
\hline$\tau_{\text {trans-cis }}$ & 1.9 & 0.42 & 0.052 & 0.059 & 0.074 & 0.017 \\
\hline$\tau_{\text {cis-cis }}$ & 31 & $5.3 \cdot 10-3$ & $2.5 \cdot 10-5$ & $5.2 \cdot 10-6$ & $9.1 \cdot 10-7$ & $1.9 \cdot 10-8$ \\
\hline$\tau_{\text {trans-trans }}$ & 190 & 0.034 & $3.1 \cdot 10-4$ & $1.4 \cdot 10-4$ & $2.6 \cdot 10-5$ & $1.9 \cdot 10-6$ \\
\hline \multicolumn{7}{|l|}{$\mathrm{t}, \mathrm{c}$} \\
\hline$t_{\text {trans }}$ & $3.8 \cdot 10-3$ & 2.7 & 5.9 & 7.1 & 10.0 & \multirow{2}{*}{$11.0^{* *}$} \\
\hline $\mathrm{t}_{\text {cis }}$ & $6.7 \cdot 10-3$ & 0.088 & 0.44 & 0.69 & 0.84 & \\
\hline
\end{tabular}




\section{References}

1. DeKock, R. L.; McGuire, M. J.; Piecuch, P.; Allen, W. D.; Schaefer, H. F.; Kowalski, K.; Kucharski, S. A.; Musiał, M.; Bonner, A. R.; Spronk, S. A., et al. The Electronic Structure and Vibrational Spectrum of trans-HNOO. J. Phys. Chem. A 2004, 108, 2893-2903.

2. Talipov, M. R.; Khursan, S. L.; Safiullin, R. L. RRKM and Ab Initio Investigation of the NH (X) Oxidation by Dioxygen. J. Phys. Chem. A 2009, 113, 6468-6476.

3. Talipov, M. R.; Khursan, S. L.; Safiullin, R. L. The Reaction of Nitroso Oxides with Olefins: Concerted or Nonconcerted Addition? . Russ. J. Phys. Chem. A 2011, 85, 364-376. 